\title{
Coupled equations for Kähler metrics and Yang-Mills connections
}

\author{
LUIS ÁLVAREZ-CÓNSUL \\ MARIO GARCÍA-FERNÁNDEZ \\ OSCAR GARCÍA-PRADA
}

\begin{abstract}
We study equations on a principal bundle over a compact complex manifold coupling a connection on the bundle with a Kähler structure on the base. These equations generalize the conditions of constant scalar curvature for a Kähler metric and HermiteYang-Mills for a connection. We provide a moment map interpretation of the equations and study obstructions for the existence of solutions, generalizing the Futaki invariant, the Mabuchi K-energy and geodesic stability. We finish by giving some examples of solutions.
\end{abstract}

32Q20, 53C07

\section{Introduction}

In this paper we consider a system of partial differential equations coupling a Kähler metric on a compact complex manifold and a connection on a principal bundle over it. These equations, inspired by the Hitchin-Kobayashi correspondence for bundles and the Yau-Tian-Donaldson conjecture for constant scalar curvature Kähler (cscK) metrics, intertwine the curvature of a Hermitian-Yang-Mills (HYM) connection on the bundle and the scalar curvature of a Kähler metric on the manifold.

To write our equations explicitly, let $X$ be a smooth compact manifold and let $G$ be a compact real Lie group with Lie algebra $\mathfrak{g}$. Let $E$ be a principal $G$-bundle over $X$. We fix a positive definite inner product $(\cdot, \cdot)$ on $\mathfrak{g}$ invariant under the adjoint representation. Let $\Omega^{k}$ be the space of smooth $k$-forms on $X$. Considering the space $\Omega^{k}(\operatorname{ad} E)$ of smooth $k$-forms on $X$ with values in the adjoint bundle ad $E$, the inner product of $\mathfrak{g}$ induces a pairing

$$
\Omega^{p}(\operatorname{ad} E) \times \Omega^{q}(\operatorname{ad} E) \longrightarrow \Omega^{p+q},
$$

that we write simply as $a_{p} \wedge a_{q}$ for any $a_{j} \in \Omega^{j}(\operatorname{ad} E), j=p, q$. The unknown variables of the equations are a Kähler structure $(g, \omega, J)$ on the base $X$ and a connection $A$ on $E$, where $g, \omega$ and $J$ are respectively the metric, the symplectic form 
and the complex structure. We will say that a Kähler structure $(g, \omega, J)$ on $X$ and a connection $A$ on $E$ satisfy the coupled Kähler-Yang-Mills equations with coupling constants $\alpha_{0}, \alpha_{1} \in \mathbb{R}$ if:

$$
\left.\begin{array}{l}
\Lambda F_{A}=z \\
\alpha_{0} S_{g}+\alpha_{1} \Lambda^{2}\left(F_{A} \wedge F_{A}\right)=c
\end{array}\right\}
$$

Here $S_{g}$ is the scalar curvature of $g, F_{A}$ is the curvature of $A, z$ is an element of $\mathfrak{g}$ that is invariant under the adjoint $G$-action and $c$ is a real number. The precise values of $z$ and $c$ are determined by the topology of $E$, the cohomology class of $\omega$ and the coupling constants $\alpha_{0}, \alpha_{1}$ (see Remark 1.2 and (2.49)). The map $\Lambda: \Omega^{p, q}(\operatorname{ad} E) \rightarrow \Omega^{p-1, q-1}(\operatorname{ad} E)$ is the contraction operator acting on $(p, q)$-type valued forms determined by the Kähler structure. In the sequel, we will refer to (0.2) simply as the coupled equations.

A link with holomorphic geometry is provided by the additional integrability condition

$$
F_{A}^{0,2}=0
$$

between the complex structure $J$ on the base and the connection $A$. Here $F_{A}^{0,2}$ denotes the $(0,2)$ part of the curvature, regarded as an $(\mathrm{ad} E)$-valued smooth form on $X$. Let $G^{c}$ be the complexification of the group $G$. When (0.3) holds, the pair $(J, A)$ endows the associated principal $G^{c}$-bundle $E^{c}=E \times_{G} G^{c}$ with a structure of holomorphic principal bundle over the complex manifold $(X, J)$.

The moment map interpretations of the constant scalar curvature equation for a Kähler metric (cscK) and the HYM equation provide a guiding principle, leading to (0.2). Indeed, equations (0.2) have an interpretation in terms of a moment map. This is the subject of Section 1 and Section 2. As observed by Fujiki [21] and Donaldson [17], the $\operatorname{cscK}$ equation has a moment map interpretation in terms of a symplectic form $\omega$ on the smooth compact manifold $X$. The group of symmetries of the theory for cscK metrics is the group $\mathcal{H}$ of Hamiltonian symplectomorphisms. This group acts on the space $\mathcal{J}^{i}$ of integrable almost complex structures on $X$ that are compatible with $\omega$, and this action is Hamiltonian for a natural symplectic form $\omega_{\mathcal{J}}$ on $\mathcal{J}^{i}$. The moment map interpretation of the HYM equation was pointed out first by Atiyah and Bott [4] for the case of Riemann surfaces and generalized by Donaldson [15] to higher dimensions. Here one considers the symplectic action of the gauge group $\mathcal{G}$ of the bundle $E$ on the space of connections $\mathcal{A}$ endowed with a natural symplectic form $\omega_{\mathcal{A}}$. Relying on these two previous cases, the phase space for our theory is provided by the subspace of the product

$$
\mathcal{P} \subset \mathcal{J}^{i} \times \mathcal{A}
$$


defined by the condition (0.3). Our choice of symplectic structure is the restriction to $\mathcal{P}$ of the symplectic form

$$
\omega_{\alpha}=\alpha_{0} \omega_{\mathcal{J}}+4 \alpha_{1} \omega_{\mathcal{A}},
$$

for a pair of non-zero coupling constants $\alpha=\left(\alpha_{0}, \alpha_{1}\right) \in \mathbb{R}^{2}$.

Consider now the extended gauge group $\widetilde{\mathcal{G}}$ defined as the group of automorphisms of the bundle $E$ covering Hamiltonian symplectomorphisms of $X$. This is a non-trivial extension

$$
1 \longrightarrow \mathcal{G} \longrightarrow \tilde{\mathcal{G}} \longrightarrow \mathcal{H} \longrightarrow 1
$$

where $\mathcal{G}$ is the group of automorphisms of $E$ covering the identity on $X$, and $\mathcal{H}$, as above, is the group of Hamiltonian symplectomorphisms of $X$. The group $\widetilde{\mathcal{G}}$ acts on $\mathcal{P}$ and in Proposition 2.1 we show that this action is Hamiltonian for any value of the coupling constants, we compute a moment map $\mu_{\alpha}$, and show that its zero locus corresponds to solutions of $(0.2)$. The coupling between the metric and the connection occurs as a direct consequence of the structure of $\widetilde{\mathcal{G}}$. So, away from its singularities, the moduli space of solutions is given by the symplectic quotient:

$$
\mathcal{M}_{\alpha}=\mu_{\alpha}^{-1}(0) / \widetilde{\mathcal{G}}
$$

Furthermore, $\omega_{\alpha}$ is a Kähler form on $\mathcal{P}$ when $\alpha_{1} / \alpha_{0}>0$, for a natural $\tilde{\mathcal{G}}$-invariant complex structure on $\mathcal{P}$. Hence under this condition on the coupling constants, the smooth locus of the moduli space of solutions (0.7) inherits a Kähler structure.

We see that our problem merges the well-studied theories of Hermitian-Yang-Mills connections (obtained for $\alpha_{1} / \alpha_{0}>0$ ) and constant scalar curvature Kähler metrics (which correspond to $\alpha_{1} / \alpha_{0}=0$ ) into a unique theory. We thus expect the Kähler moduli spaces obtained in our symplectic reduction process to have a rich geometry and topology. In Section 2.3 we prove that (0.2) arise also as absolute minima of a purely Riemannian functional for $G$-invariant Riemannian metrics on the total space of $E$, providing a link to the classical Kaluza-Klein theory.

In Section 3, which is in some sense the heart of the paper, we undertake the study of obstructions for the existence of solutions to (0.2), generalizing the Futaki invariant, the Mabuchi K-energy and geodesic stability that appear in the $\operatorname{cscK}$ theory (Futaki [22], Mabuchi [41], and Chen [11]). We do this geometrically, by considering the following framework. We first fix a cohomology class $\Omega \in H^{2}(X, \mathbb{R})$ and a smooth principal $G^{c}$-bundle $E^{c} \rightarrow X$. Let $\Gamma$ be the Lie group given by those $G^{c}$-equivariant diffeomorphisms of $E^{c}$ that cover an element in the connected component of the identity 
of the diffeomorphism group of $X$. In Section 3.1, we associate an infinite-dimensional canonical $\Gamma$-equivariant double fibration

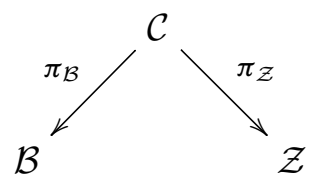

to the data $\left(X, \Omega, E^{c}\right)$. Here $\mathcal{B}$ is the space of pairs $(\omega, H)$, where $\omega \in \Omega$ is a symplectic form on $X$ and $H$ is a reduction of $E^{c}$ to the maximal compact subgroup $G \subset G^{c}$, and $\mathcal{Z}$ is a space parametrising holomorphic structures $I$ on $E^{c}$ inducing a complex structure $J$ on $X$. The space of compatible pairs $\mathcal{C} \subset \mathcal{B} \times \mathcal{Z}$ is defined as those elements of the product which induce a Kähler structure on $X$. Using the results of Section 2, in Section 3.1 we prove that the fibres of $\pi_{\mathcal{B}}$ are (formally) Kähler manifolds endowed with Hamiltonian group actions.

As a preliminary step for the study of obstructions in Section 3.3, we prove in Section 3.2 that the fibres of $\pi_{\mathcal{Z}}$ are infinite-dimensional symmetric spaces (that is, each fibre has a canonical torsion-free affine connection $\nabla$ with covariantly constant curvature), with holonomy group contained in the extended gauge group. Note that the fibre $B_{I}$ of $\pi_{\mathcal{Z}}$ over $I \in \mathcal{Z}$ is

$$
B_{I}=\mathcal{K}_{J} \times \mathcal{R}
$$

where $\mathcal{K}_{J}$ is the space of Kähler forms on $(X, J)$ in the class $\Omega$ and $\mathcal{R}$ is the space of $G$-reductions of $E^{c}$. When specialized to the case of trivial $G^{c}$, we recover the symmetric space structure constructed by Mabuchi [42] and rediscovered by Semmes [51] and Donaldson [18]. Our construction follows closely Donaldson's in [18, Section 2]. A special feature of the symmetric space structure on $B_{I}$ is that in general it does not carry any canonical compatible Riemannian structure (see Remark 4.4). A technical assumption in our construction is that the $G$-invariant metric in $\mathfrak{g}$ used to define (0.1) extends to a $G^{c}$-invariant symmetric bilinear pairing

$$
\mathfrak{g}^{c} \otimes \mathfrak{g}^{c} \longrightarrow \mathbb{C},
$$

where $\mathfrak{g}^{c}$ is the complexification of $\mathfrak{g}$.

In Section 3.3 and 3.4 we construct an $\alpha$-Futaki character

$$
\mathcal{F}_{I}: \operatorname{Lie} \operatorname{Aut}\left(E^{c}, I\right) \longrightarrow \mathbb{C},
$$

which is a complex character of the Lie algebra of the automorphism group of the holomorphic principal bundle $\left(E^{c}, I\right)$ and which vanishes when $(0.2)$ is satisfied, and 
an $\alpha-K$-energy

$$
\mathcal{M}_{I}: B_{I} \longrightarrow \mathbb{R},
$$

which is convex along geodesics on $B_{I}$ and bounded from below when (0.2) is satisfied, provided that the symmetric space $B_{I}$ is geodesically convex. Furthermore, we motivate a definition of geodesic stability of the orbit $\Gamma \cdot I$ and conjecture a link with (0.2) when $\Gamma_{I}$ is finite. We give explicit formulae for the character $\mathcal{F}_{I}$, the functional $\mathcal{M}_{I}$ and the geodesic equation on $B_{I}$. When specialized to the case in which $G^{c}$ is trivial, we recover the Futaki character [22], the Mabuchi K-energy [41] and the notion of geodesic stability $[11 ; 18]$ used in the study of the $\operatorname{cscK}$ equation for Kähler metrics. The contents of Section 3.4 will be used in Example 5.9 to provide an explicit situation in which there cannot exist solutions to the coupled equations.

We would like to point out that the framework developed in Section 3 is rather general and may be applied to other situations, in particular, to equations with a further coupling with Higgs fields.

In Section 4, we establish sufficient conditions for the existence of solutions to the coupled equations near a given solution, when the coupling constants and the Kähler cohomology class are deformed while the complex structure of the base manifold remains fixed. Our approach is based on a generalization of techniques developed by LeBrun and Simanca $[38 ; 39]$ for the corresponding problem in the cscK theory. We fix a complex structure on $X$ and a structure $I$ of a holomorphic principal $G^{c}$-bundle on $E^{c}$, and consider the space of solutions $(\omega, H)$ of $(0.2)$ with $\omega$ in a fixed cohomology class $\Omega$ and fixed $\alpha_{0}, \alpha_{1}$. Then we study the behaviour of this space with respect to deformations of the coupling constants and the Kähler class in a parameter space:

$$
\left(\alpha_{0}, \alpha_{1}, \Omega\right) \in \mathbb{R}^{2} \times H^{1,1}(X, \mathbb{R}) .
$$

Before doing this, in Section 4.1 we introduce the notion of extremal pairs $(\omega, H)$. They are analogues in our theory of Calabi's extremal metrics in Kähler geometry. In particular, an extremal pair $(\omega, H)$, with $\omega \in \Omega$, is a solution of (0.2) if and only if the $\alpha$-Futaki character associated to $I$ and $\Omega$ vanishes (Proposition 4.2). In Section 4.3 we study the linearization of (0.2) and in Section 4.4 we prove that when $\alpha_{1} / \alpha_{0}>0$, any solution of the coupled equations (0.2) can be deformed into an extremal pair, for small deformations in the parameter space (0.10) (Theorem 4.10). In Section 4.5 we obtain a criterion for the existence of solutions of (0.2) in the weak coupling limit $\alpha_{1} \rightarrow 0$, ie, for $0<\left|\alpha_{1} / \alpha_{0}\right| \ll 1$ (Theorem 4.18).

In Section 5 we discuss some examples of solutions of (0.2) and explain how the existence of solutions to the limit case $\alpha_{0}=0$ can be applied, using results of Y J Hong in [30], to obtain cscK metrics on ruled manifolds. As for the examples, in Section 5.1 we 
deal with the case of vector bundles over Riemann surfaces and projectively flat bundles over Kähler manifolds satisfying a topological constraint. In both situations, the coupled system (0.2) reduces to the limit case $\alpha_{1}=0$ ( $\operatorname{cscK}$ equation and HYM equation). When $\operatorname{dim}_{\mathbb{C}} X>1$, we use Theorem 4.10 to deform the Kähler class and provide non-trivial examples of solutions. In Section 5.2 we consider homogeneous Hermitian holomorphic vector bundles over homogeneous Kähler manifolds. In Section 5.3 we discuss some (well known) examples of stable bundles over Kähler-Einstein manifolds where Theorem 4.18 applies. Section 5.3 provides examples of solutions in which the Kähler metric is not cscK and also examples where the invariant $\mathcal{F}_{I}$ obstructs the existence of solutions for small ratio of the coupling constants.

Coupled equations for metrics and connections have of course been studied for a long time in the context of unified field theories in physics and more recently in string theory (see eg Li and Yau [40]). They have also been considered in the context of Riemannian geometry, like the Einstein-Maxwell equations on 4-manifolds studied in LeBrun [37]. Our motivation, however, for this work has been to find a Kähler analogue of these situations. Another important motivation for us comes from the relation with algebraic geometry, in particular with the moduli problem for pairs consisting of a polarised manifold and a holomorphic bundle over it. Despite its intrinsic mathematical interest and its relevance in theoretical physics, the latter problem has been little explored, probably due to the hard technical difficulties that arise in the algebro-geometric approach as soon as the complex dimension of the base is greater than 1 (see Gieseker and Morrison [27], Caporaso [9], and Pandharipande [46] for the case of curves, and Huang [31], and Schumacher and Toma [50] for higher dimensions). Throughout this paper we hope to show that the study of our coupled equations provides a reasonable differential-geometric approach to the moduli problem for bundles and varieties, giving compelling evidence of the existence of a Hitchin-Kobayashi correspondence for the coupled equations as has been conjectured in García-Fernández [25].

Since this paper was finished, there have been several developments in the theory of the coupled Kähler-Yang-Mills equations. Keller and Tønnesen-Friedman [32] have found solutions on line bundles over complex threefolds (with positive ratio $\alpha>0$ ) that do not admit any cscK metric in the class of the polarisation. The second author, jointly with C Tipler, has recently found new examples of solutions [26], by deformation of the holomorphic structure on a homogeneous bundle over $\mathbb{P}^{1} \times \mathbb{P}^{1}$. More remarkably, an interesting relation between the coupled equations and physical equations, describing gravitating vortices over a Riemann surface, has been recently found by the authors [2]. These vortices represent the coupling of gravity and a condensed matter system and are known in the physics literature as cosmic strings (or topological defects) in the Abelian Higgs model. Based on classical results by Y Yang [60], this relation provides 
a plethora of solutions of the coupled equations in $\mathbb{P}^{1} \times \mathbb{P}^{1}$ and a explicit (conjectural) description of the moduli space (0.7) and the stability condition for this particular case.

\section{Acknowledgements}

We want to thank Olivier Biquard, David Calderbank, Simon Donaldson, Nigel Hitchin, Julien Keller, Alastair King, Ignasi Mundet i Riera, Vicente Muñoz, Julius Ross, Ignacio Sols, Jacopo Stoppa and Richard Thomas for helpful discussions and suggestions. We also wish to thank the Max Planck Institute for Mathematics (Bonn), and the Isaac Newton Institute for Mathematical Sciences for their hospitality and support. MGF thanks Instituto de Ciencias Matemáticas (Madrid), Imperial College (London), University of Paris 6 and Humboldt University (Berlin) for their hospitality.

The first and the third authors are partially supported by the Spanish Ministerio de Ciencia e Innovación (MICINN) under grant MTM2010-17717. The initial work of LAC was supported by the Spanish "Programa Ramón y Cajal". Partial support of LAC was also provided by CSIC research grant 200950I027. The initial work of MGF was supported by an I3P grant of the Consejo Superior de Investigaciones Científicas. Subsequent support of MGF was provided by QGM (Centre for Quantum Geometry of Moduli Spaces), funded by the Danish National Research Foundation and by the EPFL (École Polytechnique Fédéral de Lausanne). MGF wishes also to thank the Max Planck Institute for Mathematics in Bonn (which he was visiting while part of this research was carried out) and the SFB 647 project (Humboldt University, Berlin) for financial support.

\section{Hamiltonian action of the extended gauge group}

In this section we define the extended gauge group $\tilde{\mathcal{G}}$ of a bundle over a compact symplectic manifold, an extension of the infinite-dimensional Lie groups involved in the moment map problems for the HYM and the $\operatorname{cscK}$ equation. We show that the action of $\widetilde{\mathcal{G}}$ on the space of connections of the bundle is Hamiltonian and compute an equivariant moment map. Symplectic reductions by Lie group extensions have been studied in the literature in various degrees of generality (see Marsden, Misiołek, Ortega, Perlmutter and Ratiu [43] and references therein). Previous work includes split group extensions and more general ones, although it seems that the moment map calculations of Section 1.3, based on Proposition 1.3, have not been previously made (cf [43, Section 3.2]). 


\subsection{The Hermitian-Yang-Mills equation}

First we set out some notation in order to review the moment map interpretations of the HYM equation. Let $X$ be a compact symplectic manifold of dimension $2 n$, with symplectic form $\omega, G$ a real compact Lie group with Lie algebra $\mathfrak{g}$, and $E$ a smooth principal $G$-bundle over $X$, with the $G$-action on the right. In the sequel $\omega^{[k]}$ will denote $\omega^{k} / k$ !. The spaces of smooth $k$-forms on $X$ and smooth $k$-forms with values in any given vector bundle $F$ on $X$ are denoted by $\Omega^{k}$ and $\Omega^{k}(F)$, respectively. Fix a positive definite inner product on $\mathfrak{g}$, invariant under the adjoint action, denoted

$$
(\cdot, \cdot): \mathfrak{g} \otimes \mathfrak{g} \longrightarrow \mathbb{R}
$$

This product induces a metric on the adjoint bundle ad $E=E \times_{G} \mathfrak{g}$, which extends to a bilinear map on (ad $E$ )-valued differential forms (we use the same notation as in [4, Section 3])

$$
\Omega^{p}(\operatorname{ad} E) \times \Omega^{q}(\operatorname{ad} E) \longrightarrow \Omega^{p+q}: \quad\left(a_{p}, a_{q}\right) \longmapsto a_{p} \wedge a_{q} .
$$

We consider the operator

$$
\begin{array}{r}
\Lambda=\Lambda_{\omega}: \Omega^{k} \longrightarrow \Omega^{k-2}, \\
\left.\psi \longmapsto \omega^{\sharp}\right\lrcorner \psi,
\end{array}
$$

where $\sharp$ is the operator acting on $k$-forms induced by the symplectic duality $\sharp: T^{*} X \rightarrow$ $T X$ and $\lrcorner$ denotes the contraction operator. Its linear extension to $\Omega^{k}(\operatorname{ad} E)$ is also denoted $\Lambda: \Omega^{k}(\operatorname{ad} E) \rightarrow \Omega^{k-2}(\operatorname{ad} E)$ (we use the same notation as, eg, in [15]).

Let $\mathcal{A}$ be the set of connections on $E$. This is an affine space modelled on $\Omega^{1}(\operatorname{ad} E)$, with a left action of the gauge group $\mathcal{G}$ of $E$, ie, the group of $G$-equivariant diffeomorphisms of $E$ covering the identity map on $X$. The 2-form on $\mathcal{A}$ defined by

$$
\omega_{\mathcal{A}}(a, b)=\int_{X} a \wedge b \wedge \omega^{[n-1]}
$$

for $a, b \in T_{A} \mathcal{A}=\Omega^{1}(\operatorname{ad} E), A \in \mathcal{A}$, is a $\mathcal{G}$-invariant symplectic form. As observed by Atiyah and Bott [4] when $X$ is a Riemann surface and by Donaldson [15; 16] in higher dimensions, the $\mathcal{G}$-action on $\mathcal{A}$ is Hamiltonian, with equivariant moment map $\mu_{\mathcal{G}}: \mathcal{A} \rightarrow(\operatorname{Lie} \mathcal{G})^{*}$ given by

$$
\left\langle\mu_{\mathcal{G}}(A), \zeta\right\rangle=\int_{X} \zeta \wedge\left(\Lambda F_{A}-z\right) \omega^{[n]},
$$


for $A \in \mathcal{A}, \zeta \in \operatorname{Lie} \mathcal{G}=\Omega^{0}(\operatorname{ad} E)$, where $F_{A} \in \Omega^{2}(\operatorname{ad} E)$ is the curvature of $A \in \mathcal{A}$ and $z$ is an element of the space

$$
\mathfrak{z}=\mathfrak{g}^{G}
$$

of elements of $\mathfrak{g}$ that are invariant under the adjoint $G$-action, that we identify with sections of ad $E$. Recall that the moment map satisfies

$$
\left.d\left\langle\mu_{\mathcal{G}}, \zeta\right\rangle=Y_{\zeta}\right\lrcorner \omega_{\mathcal{A}}
$$

for all $\zeta \in \operatorname{Lie} \mathcal{G}$, where $Y_{\zeta}$ is the vector field on $\mathcal{A}$ generated by the infinitesimal action of $\zeta$, and equivariance means that, for all $g \in \mathcal{G}$ and $A \in \mathcal{A}$,

$$
\mu_{\mathcal{G}}(g \cdot A)=\operatorname{Ad}\left(g^{-1}\right)^{*} \mu_{\mathcal{G}}(A) .
$$

Suppose now that $X$ is a Kähler manifold, with Kähler form $\omega$ and complex structure $J$. Consider the complexification $G^{c}$ of $G$ and the associated principal $G^{c}$-bundle $E^{c}=E \times_{G} G^{c}$, where $G$ acts on $G^{c}$ by left multiplication. There is a distinguished $\mathcal{G}$-invariant subspace

$$
\mathcal{A}_{J}^{1,1} \subset \mathcal{A}
$$

consisting of connections $A$ with $F_{A} \in \Omega_{J}^{1,1}(\operatorname{ad} E)$, or equivalently satisfying $F_{A}^{0,2}=0$, where $\Omega_{J}^{p, q}(\operatorname{ad} E)$ denotes the space of $(\operatorname{ad} E)$-valued smooth $(p, q)$-forms with respect to $J$ and $F_{A}^{0,2}$ is the projection of $F_{A}$ into $\Omega_{J}^{0,2}(\operatorname{ad} E)$. This space is in bijection with the space of holomorphic structures on the principal $G^{c}$-bundle $E^{c}$ over the complex manifold $(X, J)$ (see Singer [52]).

Definition 1.1 A connection $A \in \mathcal{A}_{J}^{1,1}$ is called Hermitian-Yang-Mills if it satisfies the Hermitian-Yang-Mills equation

$$
\Lambda F_{A}=z
$$

Remark 1.2 The element $z \in \mathfrak{z}$ in the right-hand side of (1.17) is determined by the cohomology class $\Omega:=[\omega] \in H^{2}(X)$ and the topology of the principal bundle $E$. This follows after applying $\left(z_{j}, \cdot\right)$ to (1.17), for an orthonormal basis $\left\{z_{j}\right\}$ of $\mathfrak{z} \subset \mathfrak{g}$, and then integrating over $X$, we obtain

$$
z=\sum_{j} \frac{\left\langle z_{j}(E) \cup \Omega^{[n-1]},[X]\right\rangle}{\operatorname{Vol}_{\Omega}} z_{j}
$$

Here, $\Omega^{[k]}:=\Omega^{k} / k !, \operatorname{Vol}_{\Omega}:=\int_{X} \omega^{[n]}=\left\langle\Omega^{[n]},[X]\right\rangle$ and $z_{j}(E):=\left[z_{j} \wedge F_{A}\right] \in H^{2}(X)$ is the Chern-Weil class associated to the $G$-invariant linear form $\left(z_{j}, \cdot\right)$ on $\mathfrak{g}$, which 
only depends on the topology of the bundle $E$ (see Kobayashi and Nomizu [36, Ch XII, Section 1]).

The moduli space of Hermitian-Yang-Mills connections is defined as the set of classes of gauge equivalent solutions to (1.17). This coincides with the quotient

$$
\mu_{\mathcal{G}}^{-1}(0) / \mathcal{G},
$$

where $\mu_{\mathcal{G}}$ is now the restriction of the moment map to $\mathcal{A}_{J}^{1,1}$. Away from its singularities, $\mathcal{A}_{J}^{1,1}$ inherits a complex structure compatible with $\omega_{\mathcal{A}}$ and hence a Kähler structure. Thus the smooth locus of $\mathcal{A}_{J}^{1,1}$ is a Kähler manifold endowed with a Hamiltonian $\mathcal{G}$-action and hence, away from singularities, the moduli space of Hermitian-YangMills connections can be constructed as a Kähler reduction, which, if non-empty, is a finite-dimensional Kähler manifold.

\subsection{Hamiltonian actions of extended Lie groups}

Consider a general extension of Lie groups

$$
1 \longrightarrow \mathcal{G} \stackrel{\iota}{\longrightarrow} \tilde{\mathcal{G}} \stackrel{p}{\longrightarrow} \mathcal{H} \longrightarrow 1 .
$$

We will describe now, under certain assumptions, the Hamiltonian action of $\widetilde{\mathcal{G}}$ on a symplectic manifold, in terms of $\mathcal{G}$ and $\mathcal{H}$. In the next section we will apply this general set up to the case in which the symplectic manifold is the space of connections of a bundle and $\widetilde{\mathcal{G}}$ is the extended gauge group mentioned in the introduction; this may explain the notation.

The extension (1.20) determines an extension of Lie algebras

$$
0 \longrightarrow \text { Lie } \mathcal{G} \stackrel{\iota}{\longrightarrow} \text { Lie } \tilde{\mathcal{G}} \stackrel{p}{\longrightarrow} \text { Lie } \mathcal{H} \longrightarrow 0,
$$

where the use of the same symbols $\iota$ and $p$ should lead to no confusion. Note that the short exact sequence (1.21) does not generally split as a sequence of Lie algebras, but it always does as a short exact sequence of vector spaces. Let $W \subset \operatorname{Hom}(\operatorname{Lie} \widetilde{\mathcal{G}}$, Lie $\mathcal{G})$ be the affine space of vector space splittings. Since $\mathcal{G} \subset \widetilde{\mathcal{G}}$ is a normal subgroup, there is a well-defined $\widetilde{\mathcal{G}}$-action on $W$, given by

$$
g \cdot \theta:=\operatorname{Ad}(g) \circ \theta \circ \operatorname{Ad}\left(g^{-1}\right) \text { for } g \in \tilde{\mathcal{G}}, \theta \in W .
$$

Let $\mathcal{A}$ be a manifold with an action of the "extended" Lie group $\widetilde{\mathcal{G}}$. Suppose that there exists a $\widetilde{\mathcal{G}}$-equivariant smooth map $\theta: \mathcal{A} \rightarrow W$. Let $\omega_{\mathcal{A}}$ be a symplectic form on $\mathcal{A}$ preserved by the $\tilde{\mathcal{G}}$-action. Using $\theta$, we will characterise the existence of a $\widetilde{\mathcal{G}}$-equivariant moment map for this action in terms of $\mathcal{G}$ and $\mathcal{H}$. The case considered 
in this paper (see Section 1.3) is an example where such a $\theta$ exists. Observe that if $\mathcal{A}$ is a point, then $\theta$ determines an isomorphism Lie $\widetilde{\mathcal{G}} \cong$ Lie $\mathcal{G} \rtimes$ Lie $\mathcal{H}$, which shows that in this case the existence of $\theta$ is a very strong condition.

Suppose that the $\widetilde{\mathcal{G}}$-action is Hamiltonian, with $\widetilde{\mathcal{G}}$-equivariant moment map $\mu_{\mathcal{G}}: \mathcal{A} \rightarrow$ $(\text { Lie } \widetilde{\mathcal{G}})^{*}$. We can use $\theta$ to decompose this map into two pieces corresponding to Lie $\mathcal{G}$ and Lie $\mathcal{H}$. Consider $\theta^{\perp}$ uniquely defined by $\operatorname{Id}-\iota \circ \theta=\theta^{\perp} \circ p$, where $\iota$ and $p$ are given in (1.20). Then the map

$$
\begin{aligned}
& W \longrightarrow \operatorname{Hom}(\text { Lie } \mathcal{H}, \text { Lie } \widetilde{\mathcal{G}}), \\
& \theta \longmapsto \theta^{\perp},
\end{aligned}
$$

is $\tilde{\mathcal{G}}$-equivariant, where the $\tilde{\mathcal{G}}$-action on $\operatorname{Hom}(\operatorname{Lie} \mathcal{H}$, Lie $\widetilde{\mathcal{G}})$ is given by

$$
g \cdot \theta^{\perp}=\operatorname{Ad}(g) \circ \theta^{\perp} \circ \operatorname{Ad}\left(p\left(g^{-1}\right)\right)
$$

for $g \in \tilde{\mathcal{G}}$. Moreover, the map

$$
\theta^{\perp}: \mathcal{A} \longrightarrow \operatorname{Hom}(\text { Lie } \mathcal{H} \text {, Lie } \tilde{\mathcal{G}})
$$

is $\tilde{\mathcal{G}}$-equivariant and we can decompose the moment map as

$$
\left\langle\mu_{\tilde{\mathcal{G}}}, \zeta\right\rangle=\left\langle\mu_{\tilde{\mathcal{G}}}, \iota \theta \zeta\right\rangle+\left\langle\mu_{\tilde{\mathcal{G}}}, \theta^{\perp} p(\zeta)\right\rangle,
$$

for all $\zeta \in$ Lie $\tilde{\mathcal{G}}$, where the summands in the right hand side define a pair of $\widetilde{\mathcal{G}}-$ equivariant maps $\mu_{\mathcal{G}}: \mathcal{A} \rightarrow(\operatorname{Lie} \mathcal{G})^{*}, \sigma_{\theta}: \mathcal{A} \rightarrow(\text { Lie } \mathcal{H})^{*}$, given by

$$
\begin{aligned}
\left\langle\mu_{\mathcal{G}}, \zeta\right\rangle:=\left\langle\mu_{\tilde{\mathcal{G}}}, l \zeta\right\rangle & \text { for all } \zeta \in \operatorname{Lie} \mathcal{G}, \\
\left\langle\sigma_{\theta}, \eta\right\rangle:=\left\langle\mu_{\tilde{\mathcal{G}}}, \theta^{\perp} \eta\right\rangle & \text { for all } \eta \in \operatorname{Lie} \mathcal{H} .
\end{aligned}
$$

Note that since $\mathcal{G}$ is a normal subgroup of $\widetilde{\mathcal{G}}$, we can require the map $\mu_{\mathcal{G}}$ to be $\widetilde{\mathcal{G}}$-equivariant. It is now straightforward from the moment map condition for $\mu_{\tilde{\mathcal{G}}}$ to check that $\mu_{\mathcal{G}}$ is a moment map for the $\mathcal{G}$-action on $\mathcal{A}$, ie, $\left.d\left\langle\mu_{\mathcal{G}}, \zeta\right\rangle=Y_{\zeta}\right\lrcorner \omega_{\mathcal{A}}$ for all $\zeta \in \operatorname{Lie} \mathcal{G}$. In order to see that $\sigma_{\theta}$ satisfies a similar infinitesimal condition, giving our characterization of Hamiltonian $\widetilde{\mathcal{G}}$-action, we first introduce some notation. Given a smooth map $\zeta: \mathcal{A} \rightarrow$ Lie $\widetilde{\mathcal{G}}, Y_{\zeta}$ denotes the vector field on $\mathcal{A}$ given by

$$
Y_{\zeta \mid A}:=\frac{d}{d t}{ }_{\mid t=0} \exp \left(t \zeta_{A}\right) \cdot A,
$$

for all $A \in \mathcal{A}$. In particular, $\theta: \mathcal{A} \rightarrow W$ induces a map

$$
\begin{aligned}
Y_{\theta^{\perp}}: \text { Lie } \mathcal{H} & \longrightarrow \Omega^{0}(T \mathcal{A}), \\
\eta & \longmapsto Y_{\theta^{\perp} \eta} .
\end{aligned}
$$


Note also that, by definition, $d \theta$ is a $\widetilde{\mathcal{G}}$-invariant $\operatorname{Hom}($ Lie $\mathcal{H}$, Lie $\mathcal{G})$-valued 1 -form on $\mathcal{A}$.

Proposition 1.3 The $\tilde{\mathcal{G}}$-action on $\mathcal{A}$ is Hamiltonian if and only if the action of $\mathcal{G} \subset \widetilde{\mathcal{G}}$ on $\mathcal{A}$ is Hamiltonian, with a $\widetilde{\mathcal{G}}$-equivariant moment map $\mu_{\mathcal{G}}: \mathcal{A} \rightarrow(\text { Lie } \mathcal{G})^{*}$, and there exists a smooth $\tilde{\mathcal{G}}$-equivariant map $\sigma_{\theta}: \mathcal{A} \rightarrow(\text { Lie } \mathcal{H})^{*}$ satisfying

$$
\left.Y_{\theta}{ }_{\eta}\right\lrcorner \omega_{\mathcal{A}}=\left\langle\mu_{\mathcal{G}},\langle d \theta, \eta\rangle\right\rangle+d\left\langle\sigma_{\theta}, \eta\right\rangle,
$$

for all $\eta \in$ Lie $\mathcal{H}$. In this case, a $\widetilde{\mathcal{G}}$-equivariant moment map $\mu_{\widetilde{\mathcal{G}}}: \mathcal{A} \rightarrow(\text { Lie } \widetilde{\mathcal{G}})^{*}$ is given by

$$
\left\langle\mu_{\tilde{\mathcal{G}}}, \zeta\right\rangle=\left\langle\mu_{\mathcal{G}}, \theta \zeta\right\rangle+\left\langle\sigma_{\theta}, p(\zeta)\right\rangle \quad \text { for all } \zeta \in \text { Lie } \tilde{\mathcal{G}}
$$

Proof To prove the "only if" part it remains to check (1.24). This follows by definition, differentiating in (1.22) and using that

$$
\begin{aligned}
& d\left\langle\mu_{\mathcal{G}}, \theta \zeta\right\rangle=\left\langle d \mu_{\mathcal{G}}, \theta \zeta\right\rangle+\left\langle\mu_{\mathcal{G}},\langle d \theta, \eta\rangle\right\rangle, \\
& \left.\left.\left.Y_{\zeta}\right\lrcorner \omega=Y_{\theta \zeta}\right\lrcorner \omega+Y_{\theta}{ }_{\eta}\right\lrcorner \omega \quad \text { with } \eta:=p(\zeta) \text {, }
\end{aligned}
$$

where the first equation is obtained applying the chain rule, and the second one holds because $\zeta=\theta \zeta+\theta^{\perp} \eta$ and $Y_{\zeta}$ is linear in $\zeta$. The "if" part is straightforward from the statement and is left to the reader.

Note that condition (1.24) for $\sigma_{\theta}$ generalizes the usual infinitesimal condition $\left.Y_{\eta}\right\lrcorner \omega_{\mathcal{A}}=$ $d\left\langle\mu_{\mathcal{H}}, \eta\right\rangle(\eta \in \mathcal{H})$ for moment maps $\mu_{\mathcal{H}}$ for the induced $\mathcal{H}$-action on $\mathcal{A}$ when the Lie group extension (1.20) splits.

\subsection{The extended gauge group action on the space of connections}

We apply now the general theory developed in Section 1.2 to compute the moment map for the action of the extended gauge group of a bundle over a compact symplectic manifold, on the space of connections.

Let $X$ be a compact symplectic manifold of dimension $2 n$, with symplectic form $\omega$. Let $G$ be a Lie group and $E$ be a smooth principal $G$-bundle on $X$, with projection map $\pi: E \rightarrow X$. Let $\mathcal{H}$ be the group of Hamiltonian symplectomorphisms of $(X, \omega)$ and Aut $E$ be the group of automorphisms of the bundle $E$. Recall that an automorphism of $E$ is a $G$-equivariant diffeomorphism $g: E \rightarrow E$. Any such automorphism covers a unique diffeomorphism $\check{g}: X \rightarrow X$, ie, a unique $\check{g}$ such that $\pi \circ g=\check{g} \circ \pi$. We define the Hamiltonian extended gauge group (to which we will simply refer as extended gauge group) of $E$,

$$
\widetilde{\mathcal{G}} \subset \text { Aut } E,
$$


as the group of automorphisms that cover elements of $\mathcal{H}$. Then the gauge group of $E$, already defined in Section 1.1, is the normal subgroup $\mathcal{G} \subset \widetilde{\mathcal{G}}$ of automorphisms covering the identity.

The map $\tilde{\mathcal{G}} \stackrel{p}{\longrightarrow} \mathcal{H}$ assigning to each automorphism $g$ the Hamiltonian symplectomorphism $\check{g}$ that it covers is surjective. To show this, let $h \in \mathcal{H}$. By definition there exists a Hamiltonian isotopy $[0,1] \times X \rightarrow X:(t, x) \mapsto h_{t}(x)$ from $h_{0}=$ Id to $h_{1}=h$, which is the flow of a smooth family of vector fields $\eta_{t} \in$ Lie $\mathcal{H}$, ie, with $d h_{t} / d t=\eta_{t} \circ h_{t}$ (see eg McDuff and Salamon [45, Section 3.2]). Choose a connection $A$ on $E$. Let $\zeta_{t} \in \operatorname{Lie} \tilde{\mathcal{G}}$ be the horizontal lift to $E$ of $\eta_{t}$ given by $A$. The vector fields $\zeta_{t}$ are $G$-invariant so their time-dependent flow $g_{t}$ exists for all $t \in[0,1]$ and the $g_{t}: E \rightarrow E$ are $G$-equivariant. Since $\zeta_{t}$ is a lift of $\eta_{t}$ to $E$, its flow $g_{t}$ covers $h_{t}$ (ie, $h_{t}=\check{g}_{t}$ ), so in particular $g_{t} \in \tilde{\mathcal{G}}$ for all $t$ and $g_{1} \in \tilde{\mathcal{G}}$ covers $h=h_{1}$. Thus $p$ is surjective. We thus have an exact sequence of Lie groups

$$
1 \longrightarrow \mathcal{G} \stackrel{\iota}{\longrightarrow} \tilde{\mathcal{G}} \stackrel{p}{\longrightarrow} \mathcal{H} \longrightarrow 1,
$$

where $\iota$ is the inclusion map.

Remark 1.4 Note that the sequence (1.26) is exact even when the structure group $G$ and the base manifold $X$ are non-compact. The crucial fact is that $\mathcal{H}$ lies in the identity component of the diffeomorphism group Diff $X$ of $X$ (see Abbati, Cirelli, Manià and Michor [1] for further details).

There is an action of Aut $E$, and hence of the extended gauge group, on the space $\mathcal{A}$ of connections on $E$. To define this action, we view the elements of $\mathcal{A}$ as $G$-equivariant splittings $A: T E \rightarrow V E$ of the short exact sequence

$$
0 \longrightarrow V E \longrightarrow T E \longrightarrow \pi^{*} T X \longrightarrow 0,
$$

where $V E=\operatorname{ker} d \pi$ is the vertical bundle. Using the action of $g \in$ Aut $E$ on $T E$, its action on $\mathcal{A}$ is given by $g \cdot A:=g \circ A \circ g^{-1}$. Any such splitting $A$ induces a vector space splitting of the Atiyah short exact sequence

$$
0 \longrightarrow \operatorname{Lie} \mathcal{G} \stackrel{\iota}{\longrightarrow} \operatorname{Lie}(\text { Aut } E) \stackrel{p}{\longrightarrow} \operatorname{Lie}(\operatorname{Diff} X) \longrightarrow 0
$$

(cf [4, equation (3.4)]), where Lie(Diff $X$ ) is the Lie algebra of vector fields on $X$ and $\operatorname{Lie}($ Aut $E$ ) is the Lie algebra of $G$-invariant vector fields on $E$. This splitting is given by maps

$$
\theta_{A}: \operatorname{Lie}(\text { Aut } E) \longrightarrow \operatorname{Lie} \mathcal{G}, \quad \theta_{A}^{\perp}: \operatorname{Lie}(\operatorname{Diff} X) \longrightarrow \operatorname{Lie}(\text { Aut } E)
$$

such that $\iota \circ \theta_{A}+\theta_{A}^{\perp} \circ p=\mathrm{Id}$, where $\theta_{A}$ is the vertical projection given by $A$ and $\theta_{A}^{\perp}$ the horizontal lift of vector fields on $X$ to vector fields on $E$ given by $A$. 
Lemma 1.5 Let $A \in \mathcal{A}, \zeta \in \operatorname{Lie}($ Aut $E)$ and $\check{\zeta}:=p(\zeta) \in \operatorname{Lie}(\operatorname{Diff} X)$. Then the infinitesimal action $Y_{\zeta \mid A} \in T_{A} \mathcal{A}=\Omega^{1}(\operatorname{ad} E)$ of $\zeta$ on $A$ is given by

$$
\left.Y_{\zeta \mid A}=-d_{A}\left(\theta_{A} \zeta\right)-\check{\zeta}\right\lrcorner F_{A},
$$

where $d_{A}: \Omega^{k}(\operatorname{ad} E) \rightarrow \Omega^{k+1}(\operatorname{ad} E)$ is the covariant derivative associated to $A$.

Proof By the Leibniz rule, for all $v \in \Omega^{0}(T E)$,

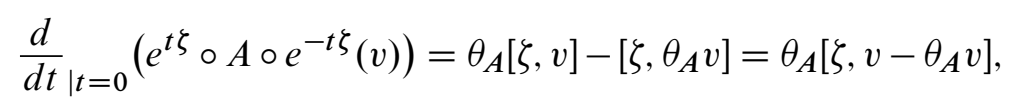

where in the second equality we have used the fact that $\zeta$ covers a vector field $\check{\zeta}$ on $X$, so that the vector field $\left[\zeta, \theta_{A} v\right]$ is vertical. It is easy to see that this expression is tensorial in $v$, so at each point of $E$ it only depends on its projection $\pi_{*} v$. Hence the vector $Y_{\zeta \mid A} \in T_{A} \mathcal{A}$, regarded as an element of $\Omega^{1}(\operatorname{ad} E)$, is given by

$$
\begin{aligned}
Y_{\zeta \mid A}(y) & =\theta_{A}\left[\zeta, \theta_{A}^{\perp} y\right]=\left[\theta_{A} \zeta, \theta_{A}^{\perp} y\right]+\theta_{A}\left[\theta_{A}^{\perp} \check{\zeta}, \theta_{A}^{\perp} y\right] \\
& \left.=\left(-d_{A}\left(\theta_{A} \zeta\right)-\check{\zeta}\right\lrcorner F_{A}\right)(y),
\end{aligned}
$$

for any $y \in \Omega^{0}(T X)$, where we have used the formulae

$$
y\lrcorner d_{A} \zeta=\left[\theta_{A}^{\perp} y, \zeta\right], \quad F_{A}\left(y, y^{\prime}\right)=-\theta_{A}\left[\theta_{A}^{\perp} y, \theta_{A}^{\perp} y^{\prime}\right]
$$

(see the equation before (4.2) and the equation after (3.4) in [4] and note that we are using a different sign convention for the curvature).

The splitting (1.29) restricts to a splitting of the exact sequence

$$
0 \longrightarrow \text { Lie } \mathcal{G} \stackrel{\iota}{\longrightarrow} \text { Lie } \tilde{\mathcal{G}} \stackrel{p}{\longrightarrow} \text { Lie } \mathcal{H} \longrightarrow 0
$$

induced by (1.26). Following the notation of Section 1.2, it is easy to see that the map

$$
\begin{aligned}
\theta: \mathcal{A} & \longrightarrow W, \\
A & \longmapsto \theta_{A},
\end{aligned}
$$

is a $\widetilde{\mathcal{G}}$-equivariant smooth map. It is also clear that the $\widetilde{\mathcal{G}}$-action on $\mathcal{A}$ is symplectic, for the symplectic form (1.13). The methods of Section 1.2 apply here to provide a moment map. To see this, we use the isomorphism of Lie algebras

$$
\text { Lie } \mathcal{H} \cong C_{0}^{\infty}(X),
$$

where Lie $\mathcal{H}$ is the Lie algebra of Hamiltonian vector fields on $X$ and $C_{0}^{\infty}(X)$ is the Lie algebra of smooth real functions on $X$ with zero integral over $X$ with respect to $\omega^{[n]}$, with the Poisson bracket. This isomorphism is induced by the map 
$C^{\infty}(X) \rightarrow$ Lie $\mathcal{H}: \phi \mapsto \eta_{\phi}$, which to each function $\phi$ assigns its Hamiltonian vector field $\eta_{\phi}$, defined by

$$
\left.d \phi=\eta_{\phi}\right\lrcorner \omega .
$$

Proposition 1.6 The $\widetilde{\mathcal{G}}$-action on $\mathcal{A}$ is Hamiltonian, with equivariant moment map $\mu_{\tilde{\mathcal{G}}}: \mathcal{A} \rightarrow(\operatorname{Lie} \widetilde{\mathcal{G}})^{*}$ given by

$$
\left\langle\mu_{\tilde{\mathcal{G}}}, \zeta\right\rangle=\left\langle\mu_{\mathcal{G}}, \theta \zeta\right\rangle+\langle\sigma, p(\zeta)\rangle \quad \text { for all } \zeta \in \operatorname{Lie} \widetilde{\mathcal{G}},
$$

where $\mu_{\mathcal{G}}: \mathcal{A} \rightarrow(\text { Lie } \mathcal{G})^{*}$ and $\sigma: \mathcal{A} \rightarrow(\text { Lie } \mathcal{H})^{*}$ are given by

$$
\begin{aligned}
\left\langle\mu_{\mathcal{G}}, \theta \zeta\right\rangle(A) & =\int_{X} \theta_{A} \zeta \wedge\left(\Lambda F_{A}-z\right) \omega^{[n]}, \\
\left\langle\sigma, \eta_{\phi}\right\rangle(A) & =-\frac{1}{4} \int_{X} \phi\left(\Lambda^{2}\left(F_{A} \wedge F_{A}\right)-4 \Lambda F_{A} \wedge z\right) \omega^{[n]},
\end{aligned}
$$

for all $A \in \mathcal{A}, \phi \in C_{0}^{\infty}(X)$.

Proof The result follows, by Proposition 1.3, from the facts that $\mu_{\mathcal{G}}$ and $\sigma$ are $\tilde{\mathcal{G}}-$ equivariant, which is immediate from (1.37) by the change of variable theorem, and the map $\sigma$ defined by (1.37) satisfies (1.24). To show this, let $\zeta \in \operatorname{Lie}(A u t E), A \in \mathcal{A}$ and note that (1.30) also applies to maps $\zeta: \mathcal{A} \rightarrow \operatorname{Lie}\left(\right.$ Aut $E$ ) (with $Y_{\zeta \mid A}$ defined by (1.23)). In particular,

$$
\left.Y_{\theta_{A}^{\perp} \eta}(A)=-\eta\right\lrcorner F_{A} \quad \text { for } \eta \in \operatorname{Lie} \mathcal{H} .
$$

The $\operatorname{Hom}($ Lie $\mathcal{H}$, Lie $\mathcal{G})$-valued 1 -form $d \theta$ on $\mathcal{A}$ is given by

$$
\begin{aligned}
d \theta(a): \text { Lie } \mathcal{H} & \longrightarrow \operatorname{Lie} \mathcal{G}, \\
\eta & \longmapsto\langle d \theta(a), \eta\rangle=a(\eta),
\end{aligned}
$$

for $A \in \mathcal{A}$ and $a \in T_{A} \mathcal{A}=\Omega^{1}(\operatorname{ad} E)$. Observe that the quantity

$$
\mu^{\prime}(A)=\int_{X}\left(\theta_{A} \zeta \wedge z-\phi \Lambda F_{A} \wedge z\right) \omega^{[n]}
$$

is locally constant on $\mathcal{A}$, so it is enough to assume $z=0$. To see this, we use the path $A_{t}=A+t a$ to calculate

$$
\begin{aligned}
\frac{d}{d t \mid t=0} \mu^{\prime}(A+t a) & =\int_{X} a\left(\eta_{\phi}\right) \wedge z \omega^{[n]}-\phi d_{A} a \wedge z \omega^{[n-1]} \\
& =\int_{X} a \wedge z d \phi \wedge \omega^{[n-1]}-\phi d_{A} a \wedge z \omega^{[n-1]}=0 .
\end{aligned}
$$


Here we have used the identity $d F_{A_{t}} / d t=d_{A} a$ for $t=0$ and integration by parts, combined with the equality

$$
a \wedge z d \phi \wedge \omega^{[n-1]}=a\left(\eta_{\phi}\right) \wedge z \omega^{[n]} .
$$

Assuming $z=0$, for the last term of the right hand side of (1.24), we have

$$
\Lambda^{2}\left(F_{A} \wedge F_{A}\right) \omega^{[n]}=2 F_{A} \wedge F_{A} \wedge \omega^{[n-2]} .
$$

Using now the Bianchi identity $d_{A} F_{A}=0$, a similar calculation as before shows that

$$
\begin{aligned}
d\langle\sigma, \eta\rangle(a) & =-\frac{1}{2} \frac{d}{d t}{ }_{\mid t=0} \int_{X} \phi\left(F_{A_{t}} \wedge F_{A_{t}}\right) \wedge \omega^{[n-2]} \\
& =-\int_{X} \phi d_{A} a \wedge F_{A} \wedge \omega^{[n-2]} \\
& \left.=\int_{X}(\eta\lrcorner \omega\right) \wedge a \wedge F_{A} \wedge \omega^{[n-2]} .
\end{aligned}
$$

To compute the integral in the last equality, note that $\left(a \wedge F_{A}\right) \wedge \omega^{n-1}=0$, so contracting with $\eta$ we obtain

$$
\left.\left.a \wedge F_{A} \wedge(\eta\lrcorner \omega\right) \wedge \omega^{[n-2]}=a(\eta) \wedge \Lambda F_{A} \omega^{[n]}-a \wedge(\eta\lrcorner F_{A}\right) \wedge \omega^{[n-1]},
$$

using the identity $F_{A} \wedge \omega^{[n-1]}=\Lambda F_{A} \omega^{[n]}$. Combined with (1.38), we thus obtain (1.24):

$$
\begin{aligned}
d\langle\sigma, \eta\rangle(a) & \left.=\int_{X} a \wedge(\eta\lrcorner F_{A}\right) \wedge \omega^{n-1}-\int_{X} a(\eta) \wedge\left(\Lambda F_{A}-z\right) \omega^{[n]} \\
& =\left(Y_{\theta_{A}} \eta^{[n} \omega_{\mathcal{A}}\right)(a)-\left\langle\mu_{\mathcal{G}},\langle d \theta(a), \eta\rangle\right\rangle .
\end{aligned}
$$

\section{The coupled equations}

In this section we give a moment map interpretation of the coupled equations (0.2) for the action of the extended gauge group, introduced in Section 1. We also define a purely Riemannian functional, the Calabi-Yang-Mills functional, whose absolute minima over the phase space are precisely the solutions of the coupled equations, that we interpret in terms of the Kaluza-Klein theory for $G$-invariant metrics on the total space of the bundle. With this purpose we first recall the moment map interpretation of the cscK equation given by Fujiki and Donaldson. 


\subsection{The Hermitian scalar curvature}

The moment map interpretation of the scalar curvature was first given by Fujiki [21] for the Riemannian scalar curvature of Kähler manifolds and generalized independently by Donaldson [17] for the Hermitian scalar curvature of almost Kähler manifolds. Here we follow closely Donaldson's approach.

First we recall the notion of Hermitian scalar curvature of an almost Kähler manifold. Fix a compact symplectic manifold $X$ of dimension $2 n$, with symplectic form $\omega$. An almost complex structure $J$ on $X$ is called compatible with $\omega$ if the bilinear form $g_{J}(\cdot, \cdot):=\omega(\cdot, J \cdot)$ is a Riemannian metric on $X$. Any almost complex structure $J$ on $X$ that is compatible with $\omega$ defines a Hermitian metric on $T^{*} X$ and there is a unique unitary connection on $T^{*} X$ whose $(0,1)$ component is the operator $\bar{\partial}_{J}: \Omega_{J}^{1,0} \rightarrow \Omega_{J}^{1,1}$ induced by $J$. The real 2 -form $\rho_{J}$ is defined as $-\mathbf{i}$ times the curvature of the induced connection on the canonical line bundle $K_{X}=\Lambda_{\mathbb{C}}^{n} T^{*} X$, where $\mathbf{i}$ is the imaginary unit $\sqrt{-1}$. The Hermitian scalar curvature $S_{J}$ is the real function on $X$ defined by

$$
S_{J} \omega^{[n]}=2 \rho_{J} \wedge \omega^{[n-1]} .
$$

The normalization is chosen so that $S_{J}$ coincides with the Riemannian scalar curvature when $J$ is integrable. The space $\mathcal{J}$ of almost complex structures $J$ on $X$ that are compatible with $\omega$ is an infinite-dimensional Kähler manifold, with complex structure $\mathbf{J}: T_{J} \mathcal{J} \rightarrow T_{J} \mathcal{J}$ and Kähler form $\omega_{\mathcal{J}}$ given by

$$
\mathbf{J} \Phi:=J \Phi \quad \text { and } \quad \omega_{\mathcal{J}}(\Psi, \Phi):=\frac{1}{2} \int_{X} \operatorname{tr}(J \Psi \Phi) \omega^{[n]},
$$

for $\Phi, \Psi \in T_{J} \mathcal{J}$, respectively. Here we identify $T_{J} \mathcal{J}$ with the space of endomorphisms $\Phi: T X \rightarrow T X$ such that $\Phi$ is symmetric with respect to the induced metric $\omega(\cdot, J \cdot)$ and satisfies $\Phi J=-J \Phi$.

The group $\mathcal{H}$ of Hamiltonian symplectomorphisms $h: X \rightarrow X$ acts on $\mathcal{J}$ by pushforward, ie, $h \cdot J:=h_{*} \circ J \circ h_{*}^{-1}$, preserving the Kähler form. As proved by Donaldson [17, Proposition 9], the $\mathcal{H}$-action on $\mathcal{J}$ is Hamiltonian with equivariant moment map $\mu_{\mathcal{H}}: \mathcal{J} \rightarrow(\text { Lie } \mathcal{H})^{*}$ given by

$$
\left\langle\mu_{\mathcal{H}}(J), \eta_{\phi}\right\rangle=-\int_{X} \phi S_{J} \omega^{[n]},
$$

for $\phi \in C_{0}^{\infty}(X)$, identified with an element $\eta_{\phi}$ in Lie $\mathcal{H}$ by (1.34) and (1.35). The $\mathcal{H}$-invariant subspace $\mathcal{J}^{i} \subset \mathcal{J}$ of integrable almost complex structures is a complex submanifold (away from its singularities), and therefore inherits a Kähler structure. Over $\mathcal{J}^{i}$, the Hermitian scalar curvature $S_{J}$ is the Riemannian scalar curvature of the 
Kähler metric determined by $J$ and $\omega$. Hence the quotient

$$
\mu_{\mathcal{H}}^{-1}(0) / \mathcal{H}
$$

where $\mu_{\mathcal{H}}$ is now the restriction of the moment map to $\mathcal{J}^{i}$, is the moduli space of Kähler metrics with fixed Kähler form $\omega$ and constant scalar curvature. Away from singularities, this moduli space can thus be constructed as a Kähler reduction (see [21] and references therein for details).

\subsection{The coupled equations as a moment map condition}

Fix a compact symplectic manifold $X$ of dimension $2 n$ with symplectic form $\omega$, a compact Lie group $G$ and a smooth principal $G$-bundle $E$ on $X$. Let $\mathcal{J}$ be the space of almost complex structures compatible with $\omega$ and $\mathcal{A}$ the space of connections on $E$. Using the symplectic forms on $\mathcal{A}$ and $\mathcal{J}$ induced by $\omega$ (see (1.13) and (2.40)), we define a symplectic form on the product $\mathcal{J} \times \mathcal{A}$, for each pair of non-zero real constants $\alpha=\left(\alpha_{0}, \alpha_{1}\right)$, as the weighted sum

$$
\omega_{\alpha}=\alpha_{0} \omega_{\mathcal{J}}+4 \alpha_{1} \omega_{\mathcal{A}}
$$

(we omit pullbacks to $\mathcal{J} \times \mathcal{A}$ ). The extended gauge group $\tilde{\mathcal{G}}$ has a canonical action on $\mathcal{J} \times \mathcal{A}$ and this action is symplectic for any $\omega_{\alpha}$. Following the notation of Section 1.3, this action is given by

$$
g \cdot(J, A)=(p(g) \cdot J, g \cdot A),
$$

for $g \in \tilde{\mathcal{G}}$ and $(J, A) \in \mathcal{J} \times \mathcal{A}$, with $p$ as in (1.26). Using the moment maps $\mu_{\mathcal{H}}$ and $\mu_{\tilde{\mathcal{G}}}$ given by (2.41) and Proposition 1.6, we obtain the following.

Proposition 2.1 The $\widetilde{\mathcal{G}}$-action on $\mathcal{J} \times \mathcal{A}$ is Hamiltonian with respect to $\omega_{\alpha}$, with equivariant moment map $\mu_{\alpha}: \mathcal{J} \times \mathcal{A} \rightarrow(\text { Lie } \widetilde{\mathcal{G}})^{*}$ given by

$$
\begin{aligned}
\left\langle\mu_{\alpha}(J, A), \zeta\right\rangle=4 \alpha_{1} & \int_{X} \theta_{A} \zeta \wedge\left(\Lambda F_{A}-z\right) \omega^{[n]} \\
& -\int_{X} \phi\left(\alpha_{0} S_{J}+\alpha_{1} \Lambda^{2}\left(F_{A} \wedge F_{A}\right)-4 \alpha_{1} \Lambda F_{A} \wedge z\right) \omega^{[n]},
\end{aligned}
$$

for all $(J, A) \in \mathcal{J} \times \mathcal{A}, \zeta \in \operatorname{Lie} \widetilde{\mathcal{G}}$, and $p(\zeta)=\eta_{\phi}$ with $\phi \in C_{0}^{\infty}(X)$.

The $\widetilde{\mathcal{G}}$-action also preserves the almost complex structure $\mathbf{I}$ on $\mathcal{J} \times \mathcal{A}$ given by

$$
\mathbf{I}(\dot{J}, a)=(J \dot{J},-a(J \cdot)),
$$

for all $(\dot{J}, a) \in T_{J} \mathcal{J} \times T_{A} \mathcal{A}$. Using the complex structure $\mathbf{J}$ on $\mathcal{J}$ given by (2.40), the canonical projection $\mathcal{J} \times \mathcal{A} \rightarrow \mathcal{J}$ becomes now a holomorphic submersion. It is 
easy to see that, for $\alpha_{0}, \alpha_{1}$ positive, the complex structure $\mathbf{I}$ is compatible with the family of symplectic structures (2.43). The formal integrability of the almost complex structure $\mathbf{I}$ is not obvious a priori, so we now provide a proof of this fact. By "formal integrability" here, we mean, as in [18], that the associated Nijenhuis tensor vanishes.

Proposition 2.2 The almost complex structure $\mathbf{I}$ is formally integrable.

Proof Since the complex structure $\mathbf{J}$ on the base $\mathcal{J}$ and the one on each fibre are integrable, the integrability condition for $\mathbf{I}$ reduces to the vanishing condition for the value of the Nijenhuis tensor $N_{\mathbf{I}}$ on each pair of vectors $j \in T_{J} \mathcal{J}, a \in T_{A} \mathcal{A}$, for $(J, A) \in \mathcal{J} \times \mathcal{A}$. Now, $a$ and $\dot{J}$ extend to vector fields on $\mathcal{A}$ and $\mathcal{J}$, respectively, and hence to $\mathcal{J} \times \mathcal{A}$ ( $a$ extends to a constant vector field on the affine space $\mathcal{A}$ and $\dot{J}$ extends to a vector field on $\mathcal{J}$ given by $\left.\dot{J}_{\mid J^{\prime}}=(1 / 2)\left(J \dot{J} J^{\prime}-J^{\prime} J \dot{J}\right)\right)$. Furthermore,

$$
N_{\mathbf{I}}(\dot{J}, a)=[\mathbf{I} \dot{J}, \mathbf{I} a]-\mathbf{I}[\mathbf{I} \dot{J}, a]-\mathbf{I}[\dot{J}, \mathbf{I} a]-[\dot{J}, a]=[\mathbf{I} \dot{J}, \mathbf{I} a]-\mathbf{I}[\dot{J}, \mathbf{I} a],
$$

where the brackets denote the Lie brackets between vector fields on $\mathcal{J} \times \mathcal{A}$ and we have used the fact that $[\mathbf{I} \dot{J}, a]=[\dot{J}, a]=0$ because the flow of $a$ covers the identity on $\mathcal{J}$. To compute the remaining terms, we denote by $J_{t}(\dot{J})$ the flow of any vector field $\dot{J}$ on $\mathcal{J}$, viewed as a vector field on $\mathcal{J} \times \mathcal{A}$. Then $J_{t}(\dot{J})$ induces the identity on $\mathcal{A}$, and hence

$$
\begin{aligned}
N_{\mathbf{I}(\dot{J}, a)} & =\frac{d}{d t}{ }_{\mid t=0} \mathbf{I} a_{\mid J_{t}(\mathbf{I} \dot{J})}-\mathbf{I}_{\mid J} \frac{d}{d t}{ }_{\mid t=0} \mathbf{I} a_{\mid J_{t}(\dot{J})} \\
& =-\left.\frac{d}{d t}\right|_{t=0} a\left(J_{t}(\mathbf{I} \dot{J}) \cdot\right)+\left.\mathbf{I}_{\mid J} \frac{d}{d t}\right|_{t=0} a\left(J_{t}(\dot{J}) \cdot\right) \\
& =-a(J \dot{J} \cdot)-a(\dot{J} J \cdot)=-a(J \dot{J}+\dot{J} J \cdot)=0,
\end{aligned}
$$

where $a$ is now viewed as an element of $\Omega^{1}(\operatorname{ad} E)$.

Note that the vanishing of $N_{\mathbf{I}}(\dot{J}, a)$ does not require any compatibility condition between $J$ and $\omega$.

Remark 2.3 There is another $\widetilde{\mathcal{G}}$-invariant almost complex structure on $\mathcal{J} \times \mathcal{A}$, which is given by $\mathbf{I}^{\prime}(\dot{J}, a)=(J \dot{J}, a(J \cdot))$. This is compatible with $\omega_{\alpha}$ for $\alpha_{0}>0>\alpha_{1}$, and the projection $\mathcal{J} \times \mathcal{A} \rightarrow \mathcal{J}$ is pseudoholomorphic for this $\mathbf{I}^{\prime}$, but one can modify the proof of Proposition 2.2 to show that $\mathbf{I}^{\prime}$ is not formally integrable.

Suppose now that $X$ has Kähler structures with Kähler form $\omega$. In the notation of Section 2.1, this means that the subspace $\mathcal{J}^{i} \subset \mathcal{J}$ of integrable almost complex structures compatible with $\omega$ is not empty. Define

$$
\mathcal{P} \subset \mathcal{J} \times \mathcal{A}
$$


as the space of pairs $(J, A)$ with $J \in \mathcal{J}^{i}$ and $A \in \mathcal{A}_{J}^{1,1}$, where $\mathcal{A}_{J}^{1,1} \subset \mathcal{A}$ is the space of connections defined in (1.16). Then $\mathcal{P} \subset \mathcal{J} \times \mathcal{A}$ is a $\widetilde{\mathcal{G}}$-invariant complex and hence Kähler subspace by construction (see also Lemma 3.1).

We say that a pair $(J, A) \in \mathcal{P}$ satisfies the coupled Kähler-Yang-Mills equations if

$$
\left.\begin{array}{l}
\Lambda F_{A}=z \\
\alpha_{0} S_{J}+\alpha_{1} \Lambda^{2}\left(F_{A} \wedge F_{A}\right)=c,
\end{array}\right\}
$$

where $S_{J}$ is the scalar curvature of the metric $g_{J}=\omega(\cdot, J \cdot)$ on $X$ and $c \in \mathbb{R}$. These equations are the central subject of this paper. The set of solutions to the coupled equations is invariant under the action of $\widetilde{\mathcal{G}}$ and we define the moduli space of solutions as the set of all solutions modulo the action of $\widetilde{\mathcal{G}}$. We have the following.

Proposition 2.4 The subset $\mu_{\alpha}^{-1}(0) \subset \mathcal{P}$ coincides with the set of pairs $(J, A) \in \mathcal{P}$ satisfying equations (2.47).

Proof Suppose that $(J, A) \in \mu_{\alpha}^{-1}(0)$. First, evaluating $\mu_{\alpha}(J, A)$ on elements of the form $\theta_{A}^{\perp} \eta$ with $\eta \in$ Lie $\mathcal{H}$, we see that there exists a $c^{\prime} \in \mathbb{R}$ such that

$$
\begin{aligned}
\frac{c^{\prime}-\alpha_{0} S_{J}}{\alpha_{1}} & =\Lambda^{2}\left(F_{A} \wedge F_{A}\right)-4 \Lambda F_{A} \wedge z \\
& =2\left|\Lambda F_{A}\right|^{2}-2\left|F_{A}\right|^{2}+8\left|F_{A}^{0,2}\right|^{2}-4 \Lambda F_{A} \wedge z,
\end{aligned}
$$

where the last equality follows from a pointwise computation (cf Mundet i Riera [49, proof of Lemma 7.9]). Here, the pointwise norms are defined using the metric $g_{J}=\omega(\cdot, J \cdot)$ and the inner product $(\cdot, \cdot)$ on $\mathfrak{g}$ and $F_{A}^{0,2}$ denotes the $(0,2)$ part of $F_{A}$ with respect to $J$. Second, as $\left\langle\mu_{\alpha}(J, A), \zeta\right\rangle=0$ for all $\zeta \in \operatorname{Lie} \mathcal{G}$, we have $\Lambda F_{A}=z$ and hence it is straightforward to see from (2.48) that

$$
\alpha_{0} S_{J}+\alpha_{1} \Lambda^{2}\left(F_{A} \wedge F_{A}\right)=c^{\prime}+4 \alpha_{1}|z|^{2} \in \mathbb{R} .
$$

The converse follows also from (2.48).

Note that we have not used the fact that $(J, A) \in \mathcal{P}$. Observe also that $c$ is a "topological constant", ie, it only depends on the cohomology class $\Omega:=[\omega] \in H^{2}(X)$, the topology of the bundle $E$ and the coupling constants $\alpha_{0}, \alpha_{1}$ (cf Remark 1.2). This follows by integrating the second equation in (2.47) over $X$, obtaining

$$
c=\alpha_{0} \widehat{S}+2 \alpha_{1} \widehat{c},
$$

where $\widehat{S}$ is the average of the Hermitian scalar curvature,

$$
\widehat{S}:=\frac{\int_{X} S_{J} \omega^{[n]}}{\int_{X} \omega^{[n]}}=2 \pi \frac{\left\langle c_{1}(X) \cup \Omega^{[n-1]},[X]\right\rangle}{\operatorname{Vol}_{\Omega}}
$$


which only depends on the cohomology class $\Omega \in H^{2}(X)$, and

$$
\hat{c}:=\frac{\int_{X} F_{A} \wedge F_{A} \wedge \omega^{[n-2]}}{\int_{X} \omega^{[n]}}=\frac{\left\langle c(E) \cup \Omega^{[n-2]},[X]\right\rangle}{\operatorname{Vol}_{\Omega}},
$$

where $c(E):=\left[F_{A} \wedge F_{A}\right] \in H^{4}(X)$ is the Chern-Weil class associated to the $G-$ invariant symmetric bilinear form $(\cdot, \cdot)$ on $\mathfrak{g}$, and so $\hat{c}$ only depends on $\Omega$ and the topology of $E$ (see [36, Ch XII, Section 1]).

From Proposition 2.4, we can identify the moduli space of solutions to the coupled equations with the quotient

$$
\mu_{\alpha}^{-1}(0) / \widetilde{\mathcal{G}},
$$

where $\mu_{\alpha}$ denotes now the restriction of the moment map to $\mathcal{P}$. Away from singularities, this is a Kähler quotient for the action of $\widetilde{\mathcal{G}}$ on the smooth part of $\mathcal{P} \subset \mathcal{J} \times \mathcal{A}$ equipped with the Kähler form obtained by the restriction of $\omega_{\alpha}$.

Remark 2.5 The coupled equations (2.47) can also be written as:

$$
\left.\begin{array}{l}
\Lambda F_{A}=z, \\
\alpha_{0} S_{g}-2 \alpha_{1}\left|F_{A}\right|_{g}^{2}=c-2 \alpha_{1}|z|^{2} .
\end{array}\right\}
$$

Here $S_{g}$ is the scalar curvature of the metric $g=\omega(\cdot, J \cdot),\left|F_{A}\right|_{g}^{2}$ is the pointwise norm of $F_{A}$ defined using $g$ and the inner product $(\cdot, \cdot)$ on $\mathfrak{g}$, and $z \in \mathfrak{z}, c \in \mathbb{R}$ are as in (2.47). The purely Riemannian nature of the second (scalar) equation in (2.53) will be used in Section 2.3. The equivalence of (2.47) and (2.53) follows from (2.48) using that $A \in \mathcal{A}_{J}^{1,1}$ (ie, $F_{A}^{0,2}=0$ ).

\subsection{The Calabi-Yang-Mills functional}

Kähler metrics of constant scalar curvature arise as the absolute minima of the Calabi functional [8], which is defined as the $L^{2}$-norm of the scalar curvature for Kähler metrics running over a fixed Kähler class on a compact complex manifold. Alternatively, we can see the cscK metrics as the absolute minima of the $L^{2}$-norm of the scalar curvature defined over the space $\mathcal{J}^{i}$ of complex structures compatible with a fixed symplectic form $\omega$ (see eg Futaki and Ono [24]). As a further step in Calabi's programme, in this section we define the Calabi-Yang-Mills (CYM) functional $\mathrm{CYM}_{\alpha}$. This is a purely Riemannian functional that intertwines the Yang-Mills functional for connections with the $L^{2}$-norm of the scalar curvature of invariant metrics in the total space of the principal bundle $E$. Interpreting the elements of $\mathcal{J} \times \mathcal{A}$ as invariant Riemannian metrics $g_{\alpha}$ on $E$, we prove that the absolute minima of $\mathrm{CYM}_{\alpha}$ over 
$\mathcal{J}^{i} \times \mathcal{A}$ are precisely the solutions $(J, A) \in \mathcal{P}$ of (2.47). We will also see that the coupled equations (2.47) can be formulated in terms of the Ricci tensor and the scalar curvature of $g_{\alpha}$, when it is defined by an element of a suitable subspace $\mathcal{P}^{*} \subset \mathcal{P}$.

We start with a principal $G$-bundle $E$ over a compact manifold $X$ and a fixed $G-$ invariant inner product $(\cdot, \cdot)$ on $\mathfrak{g}$. Consider the $G$-invariant metric $g_{V}$ on the vertical bundle $V E \subset T E$ induced by $(\cdot, \cdot)$ via the identification of $V E$ with the trivial bundle $E \times \mathfrak{g}$. Using a connection $A$ on $E$ and a scaling constant $\alpha>0$, each Riemannian metric $g$ on $X$ lifts to a $G$-invariant Riemannian metric $g_{\alpha}$ on $E$, given by

$$
g_{\alpha}=\pi^{*} g+\alpha g_{V}\left(\theta_{A} \cdot, \theta_{A} \cdot\right),
$$

where $\pi: E \rightarrow X$ is the canonical projection and $\theta_{A}: T E \rightarrow V E$ is the vertical projection determined by $A$. Given positive constants $\alpha_{0}, \alpha_{1} \in \mathbb{R}$, we denote respectively by $S_{g_{\alpha}}, \operatorname{vol}_{\alpha}$ and $\operatorname{Vol}_{\alpha}(E)$ the scalar curvature and the volume form of the metric $g_{\alpha}$ and the volume of $E$ with respect to $g_{\alpha}$, where $\alpha=2 \alpha_{1} / \alpha_{0}$. We also denote by vol $g$ and $\operatorname{Vol}_{g}(X)$ the volume form of the metric $g$ and the corresponding volume of $X$, respectively. We define the Calabi-Yang-Mills functional by the formula

$$
\mathrm{CYM}_{\alpha}(g, A):=\frac{1}{\operatorname{Vol}_{\alpha}(E)} \int_{E} S_{g_{\alpha}}^{2} \operatorname{vol}_{\alpha}+\frac{\alpha_{1}}{\operatorname{Vol}_{g}(X)} \int_{X}\left|F_{A}\right|_{g}^{2} \operatorname{vol}_{g}
$$

for pairs $(g, A)$, where $g$ is a Riemannian metric on $X, A$ is a connection on $E$ and $\left|F_{A}\right|_{g}^{2}$ is as in (2.53). Note that (2.55) is a weighted sum of the Calabi functional [8] for metrics on $E$ and the Yang-Mills functional for $E$ (see eg Donaldson and Kronheimer [20, Section 2.1.4]).

Fix now a symplectic form $\omega$ on $X$ so that $\operatorname{vol}_{g_{J}}=\omega^{[n]}$ for all $J \in \mathcal{J}$, where $g_{J}=\omega(\cdot, J \cdot)$ and $\operatorname{dim} X=2 n$. Although the functional (2.55) is well defined for arbitrary Riemannian metrics on $X$ and connections on $E$, the solutions of the coupled equations (2.47) are the absolute minima of $\mathrm{CYM}_{\alpha}$ only when this functional is restricted to metrics of the form $g=g_{J}$, where $J$ is in the space $\mathcal{J}^{i}$ of integrable almost complex structures on $X$ which are compatible with $\omega$. In other words, we consider the functional

$$
\begin{aligned}
\mathcal{J}^{i} \times \mathcal{A} & \longrightarrow \mathbb{R}, \\
(J, A) & \longmapsto \mathrm{CYM}_{\alpha}\left(g_{J}, A\right) .
\end{aligned}
$$

Proposition 2.6 If $(J, A) \in \mathcal{P}$ satisfies the coupled equations (2.47), then the pair $(J, A)$ is an absolute minimum of the functional (2.56), provided that $\alpha_{0}$ and $\alpha_{1}$ are positive and

$$
\alpha_{1}>2 \alpha \widehat{S}+\alpha^{2}\left(\widehat{c}-|z|^{2}\right)+2 s,
$$


where $\alpha=2 \alpha_{1} / \alpha_{0}, s$ is the (constant) scalar curvature of the biinvariant metric induced by $(\cdot, \cdot)$ on $G, z$ is given by (1.18) and $\widehat{S}, \widehat{c}$ are as in (2.49), with $\Omega=[\omega]$.

Proof Note first that for any metric as in $(2.54), \pi:\left(E, g_{\alpha}\right) \rightarrow(X, g)$ is a Riemannian submersion with totally geodesic fibres (see Besse [6, Theorem 9.59], where the $G-$ Riemannian manifold playing the role of the typical fibre is $G$ itself with its biinvariant metric). Then $g_{\alpha}$ has scalar curvature $S_{g_{\alpha}}=S_{\alpha} \circ \pi$, where

$$
S_{\alpha}=S_{g}-\alpha\left|F_{A}\right|_{g}^{2}+s / \alpha \in C^{\infty}(X),
$$

$S_{g}$ being the scalar curvature of $g$ (see [6, Proposition 9.70]). Here, the group is identified with the fibre $E_{x}$ over $x \in X$. Since the volume of $E_{x}$ is independent of $x$, we have

$$
\frac{1}{\operatorname{Vol}_{\alpha}(E)} \int_{E} S_{g_{\alpha}}^{2} \operatorname{vol}_{\alpha}=\frac{1}{\operatorname{Vol}_{g}(X)} \int_{X} S_{\alpha}^{2} \operatorname{vol}_{g}
$$

In particular, for $g=g_{J}$, with $J \in \mathcal{J}^{i}$, and $c^{\prime \prime}=\alpha_{0} \widehat{S}+2 \alpha_{1}\left(\widehat{c}-|z|^{2}\right)$, we obtain

$$
\begin{array}{r}
\operatorname{CYM}_{\alpha}(g, A)=\frac{\alpha_{0}^{-2}}{\operatorname{Vol}_{\Omega}}\left\|\alpha_{0} S_{g}-2 \alpha_{1}\left|F_{A}\right|_{g}^{2}-c^{\prime \prime}\right\|_{L^{2}}^{2}+\frac{\alpha_{1}}{\operatorname{Vol}_{\Omega}}\left\|F_{A}\right\|_{L^{2}}^{2} \\
+\frac{2\left(c^{\prime \prime} / \alpha_{0}+s / \alpha\right)}{\operatorname{Vol}_{\Omega}} \int_{X}\left(S_{g}-\alpha\left|F_{A}\right|_{g}^{2}-c^{\prime \prime} / \alpha_{0}\right) \operatorname{vol}_{g} \\
+\left(c^{\prime \prime} / \alpha_{0}+s / \alpha\right)^{2} \\
=\frac{\alpha_{0}^{-2}}{\operatorname{Vol}_{\Omega}}\left\|\alpha_{0} S_{g}-2 \alpha_{1}\left|F_{A}\right|_{g}^{2}-c^{\prime \prime}\right\|_{L^{2}}^{2} \\
+\frac{\alpha_{1}-2 \alpha \hat{S}-\alpha^{2}\left(\widehat{c}-|z|^{2}\right)-2 s}{\operatorname{Vol}_{\Omega}}\left\|F_{A}\right\|_{L^{2}}^{2} \\
\quad+\left(\hat{S}+\alpha\left(\widehat{c}-|z|^{2}\right)+s / \alpha\right)\left(\widehat{S}-\alpha\left(\widehat{c}-|z|^{2}+s / \alpha\right),\right.
\end{array}
$$

where the $L^{2}$-norms are defined using $g, \omega^{[n]}$ and the inner product on $\mathfrak{g}$. Note that the last summand in the right-hand side of the last equation only depends on $\alpha, s$, the cohomology class $\Omega:=[\omega]$ and the topology of the bundle $E$. The inequality (2.57) implies that the factor multiplying the Yang-Mills functional is positive, and the result follows from the alternative formulation (2.53) of the coupled equations combined with (2.48), which gives

$$
\begin{aligned}
\left\|F_{A}\right\|_{L^{2}}^{2} & =\left\|\Lambda F_{A}\right\|_{L^{2}}^{2}+4\left\|F_{A}^{0,2}\right\|_{L^{2}}^{2}-\hat{c} \operatorname{Vol}_{\Omega} \\
& =\left\|\Lambda F_{A}-z\right\|_{L^{2}}^{2}+4\left\|F_{A}^{0,2}\right\|_{L^{2}}^{2}+2\left\langle z(E) \cup \Omega^{[n-1]},[X]\right\rangle-\left(|z|^{2}+\widehat{c}\right) \operatorname{Vol}_{\Omega} .
\end{aligned}
$$

Here, $z(E):=\left[z \wedge F_{A}\right] \in H^{2}(X)$ is the Chern-Weil class associated to the $G$-invariant linear form $(z, \cdot)$ on $\mathfrak{g}$, with $z$ given by (1.18), so the last line in the previous equation 
only depends on $\Omega \in H^{2}(X)$ and the topology of the bundle $E$ (see [36, Ch. XII, Section 1]).

Remark 2.7 The inequality (2.57) imposes no restrictions on the solutions $(J, A)$ of (2.47), because any solution $(J, A)$ of (2.47) for some $\left(\alpha_{0}, \alpha_{1}\right)$ is also a solution for the constants $\left(t \alpha_{0}, t \alpha_{1}\right)$, for all $t \in \mathbb{R}$. The claim follows from the fact the RHS on (2.57) is invariant by this scaling procedure.

Remark 2.8 Fixing a complex structure on $X$, we can view $\mathrm{CYM}_{\alpha}$ as a functional on the pairs $(\omega, A)$, with $\omega$ as in the second part of Remark 2.5. Exactly as in Proposition 2.6, in this case a solution of the coupled equations is always an absolute minimum of this functional.

The coupled equations (2.47) can also be interpreted in purely Riemannian terms, considering the $G$-invariant metrics $g_{\alpha}$ on $E$ defined by (2.54). To explain this, note that given such a metric its Ricci tensor $R_{g_{\alpha}}$ decomposes as

$$
R_{g_{\alpha}}=\left(R_{g_{\alpha}}\right)_{h h}+\left(R_{g_{\alpha}}\right)_{v v}+\left(R_{g_{\alpha}}\right)_{h v},
$$

where the indices " $h$ " and " $v$ " denote the horizontal and vertical directions in $T E$ defined by the connection $A$, respectively. Let $\mathcal{P}^{*} \subset \mathcal{P}$ be the open subset of pairs $(J, A)$ with $A \in \mathcal{A}^{*}$; the open subset of $\mathcal{A}$ consisting of irreducible connections. By irreducible connection $A \in \mathcal{A}$, we mean, as in [20, Section 4.2.2], that its isotropy group $\mathcal{G}_{A}$ inside the gauge group of $E$ is minimal; the centre of $G$. Then a pair $(J, A) \in \mathcal{P}^{*}$ satisfies (2.47) if and only if the associated metric $g_{\alpha}$ satisfies the following equations.

$$
\left.\begin{array}{l}
\left(R_{g_{\alpha}}\right)_{h v}=0 \\
S_{g_{\alpha}}=\text { const. }
\end{array}\right\}
$$

We thus have an interpretation of the Kähler quotient (2.52) (with $\mu_{\alpha}$ restricted to the open subset $\mathcal{P}^{*} \subset \mathcal{P}$ ) as a moduli space of $G$-invariant metrics on the total space of $E$ satisfying (2.59). An interesting fact here is that the condition $\alpha_{1} / \alpha_{0}>0$ is needed both to have a Kähler form $\omega_{\alpha}$ on $\mathcal{P}$ given by (2.43) (see the explanation before (2.52)) and $G$-invariant Riemannian metrics $g_{\alpha}$ on $E$, as given in (2.54).

To prove the equivalence of (2.47) and (2.59) for a pair $(J, A) \in \mathcal{P}^{*}$, note that $J$ defines a structure of Kähler manifold on $(X, \omega)$. The Hermitian-Yang-Mills equation $\Lambda F_{A}=0$ for an irreducible $A \in \mathcal{A}_{J}^{1,1}$ is equivalent to the a priori weaker Yang-Mills equation $d_{A}^{*} F_{A}=0$ (see [15, Proposition 3]). This follows because if $A \in \mathcal{A}_{J}^{1,1}$ is an irreducible Yang-Mills connection, then, by the Kähler identities,

$$
d_{A} \Lambda F_{A}=0 \Longrightarrow \Lambda F_{A} \in \operatorname{Lie} \mathcal{G}_{A}=\mathfrak{z} .
$$


Therefore the first equations in (2.53) and (2.59) are equivalent because the Yang-Mills equation is equivalent to the equation $\left(R_{g_{t}}\right)_{h v}=0$ (see [6, Proposition 9.61]). Finally, the second equations in (2.53) and (2.59) are equivalent by (2.58).

Note that the system (2.59) is halfway between the Einstein equation and the constant scalar curvature equation, in the sense that

$$
g_{\alpha} \text { is an Einstein metric } \Longrightarrow g_{\alpha} \text { satisfies (2.59) } \Longrightarrow S_{g_{\alpha}}=\text { const., }
$$

for all $(J, A) \in \mathcal{P}^{*}$, as any metric $g_{\alpha}$ satisfying the Einstein equation $R_{g_{\alpha}}=\lambda g_{\alpha}$ (with $\lambda \in \mathbb{R}$ ) has constant scalar curvature.

\section{The $\alpha$-Futaki character and the $\alpha-K$-energy}

In Section 3 we construct obstructions to the existence of solution of the coupled equations, generalizing the Futaki character [22], the Mabuchi K-energy [41; 42] and the notion of geodesic stability $[11 ; 18]$ used in the cscK Theory. For this, in Sections 3.1, 3.2, 3.3, we develop an abstract framework that we apply in Section 3.4 to the study of the coupled equations.

Throughout Section 3, we fix a compact real manifold $X$, a cohomology class $\Omega \in$ $H^{2}(X, \mathbb{R})$, a reductive complex Lie group $G^{c}$ with Lie algebra $\mathfrak{g}^{c}$, a maximal compact Lie subgroup $G \subset G^{c}$ with Lie algebra $\mathfrak{g}$ and a smooth principal $G^{c}$-bundle $\pi: E^{c} \rightarrow$ $X$. We also fix $z \in \mathfrak{z}$ as in (1.18). We assume that the space of Kähler forms in $\Omega$ is non-empty.

\subsection{Invariant Hamiltonian Kähler fibrations}

In Section 3.1, we will associate to the data $\left(X, \Omega, E^{c}\right)$ a canonical infinite-dimensional double fibration

$$
\mathcal{B} \stackrel{\pi_{\mathcal{B}}}{\longleftarrow} \mathcal{C} \stackrel{\pi_{\mathcal{Z}}}{\longrightarrow} \mathcal{Z},
$$

equivariant for the action of an infinite-dimensional Lie group $\Gamma$, and show that the fibres of $\pi_{\mathcal{B}}$ are (formally) Kähler manifolds with Hamiltonian group actions. The fibres of $\pi_{\mathcal{Z}}$ will be studied in Section 3.2.

Let $\operatorname{Diff}_{0} X$ be the identity component of the diffeomorphism group of $X$ and Aut $E^{c}$ the group of automorphisms of $E^{c}$, that is, the $G^{c}$-equivariant diffeomorphisms $g: E^{c} \rightarrow E^{c}$. Any such $g$ determines a unique diffeomorphism $\stackrel{g}{g}: X \rightarrow X$ such that $\pi \circ g=\check{g} \circ \pi$. Define the real Lie group

$$
\Gamma:=\left\{g \in \text { Aut } E^{c} \mid \stackrel{g}{g} \in \operatorname{Diff}_{0} X\right\} .
$$


Note that the Lie bracket in the Lie algebra Lie $\Gamma$ of $\Gamma$ is

$$
\left[y, y^{\prime}\right]_{\Gamma}=-\left[y, y^{\prime}\right]
$$

for $y, y^{\prime} \in$ Lie $\Gamma \subset \Omega^{0}\left(T E^{c}\right)$, where $[\cdot, \cdot]$ is the Lie bracket of vector fields on $E^{c}$ (cf [45, Remark 3.3]).

Let $\mathcal{Z}$ be the space of holomorphic structures on the principal $G^{c}$-bundle $E^{c}$, ie, the integrable $G^{c}$-equivariant almost complex structures $I$ on the total space of $E^{c}$ that preserve the vertical bundle $V E^{c}$ and whose restriction to $V E^{c}$ equals multiplication by $\sqrt{-1}$, via its identification with $E^{c} \times \mathfrak{g}^{c}$. By $G^{c}$-equivariance, any such $I$ determines a unique integrable almost complex structure $\check{I}$ on $X$ such that $\check{I} \circ d \pi=d \pi \circ I$. The group $\Gamma$ has a left action on $\mathcal{Z}$ by push-forward, preserving the canonical almost complex structure $\mathbf{I}$ on $\mathcal{Z}$ given by

$$
\mathbf{I} \dot{I}=I \dot{I} \quad \text { for all } I \in \mathcal{Z}, \dot{I} \in T_{I} \mathcal{Z}
$$

(cf (2.40)), where $\dot{I}$ is viewed as a $G^{c}$-equivariant endomorphism of $T E^{c}$.

Recall that the space $\mathcal{R}=\Omega^{0}\left(E^{c} / G\right)$ of smooth sections $H$ of the bundle $E^{c} / G \rightarrow X$ is in bijection with the set of reductions of $E^{c}$ to principal $G$-bundles $E_{H} \subset E^{c}$, via the map $H \mapsto E_{H}:=p_{G}^{-1}(H(X))$, where $p_{G}$ is the projection $E^{c} \rightarrow E^{c} / G$. Let $\mathcal{B}$ be the space of pairs $(\omega, H)$, where $\omega \in \Omega$ is a symplectic form and $H \in \mathcal{R}$. The group $\Gamma$ has a left action on $\mathcal{B}$ given by

$$
g \cdot(\omega, H)=\left(\check{g}_{*} \omega, g \cdot H\right),
$$

where $(g \cdot H)(x):=g(x) \cdot H\left(\check{g}^{-1}(x)\right)$ for $x \in X$ and $\check{g}_{*} \omega \in \Omega$ by the homotopy invariance of the de Rham cohomology, as $\check{g} \in \operatorname{Diff}_{0} X$.

We define the space of compatible pairs as

$$
\mathcal{C}:=\{((\omega, H), I) \mid(X, \check{I}, \omega) \text { is a Kähler manifold }\} \subset \mathcal{B} \times \mathcal{Z} .
$$

Note that this space is invariant under the diagonal $\Gamma$-action on $\mathcal{B} \times \mathcal{Z}$. The canonical maps

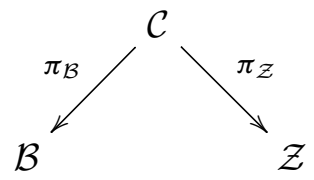

will be viewed as two fibrations with total space $\mathcal{C}$, whose fibres are

$$
Z_{b}:=\pi_{\mathcal{B}}^{-1}(b) \quad \text { and } \quad B_{I}:=\pi_{\mathcal{Z}}^{-1}(I) \text { for all } b \in \mathcal{B}, I \in \mathcal{Z} .
$$


Since $\mathcal{C} \subset \mathcal{B} \times \mathcal{Z}$ is $\Gamma$-invariant, the fibres $B_{I}$ and $Z_{b}$ are invariant under the actions of the isotropy groups $\Gamma_{I} \subset \Gamma$ and $\Gamma_{b} \subset \Gamma$, respectively.

In more concrete terms, for any $I \in \mathcal{Z}$, the isotropy group $\Gamma_{I}$ is the group of automorphisms $g$ of the holomorphic principal $G^{c}$-bundle $\left(E^{c}, I\right)$ such that $\breve{g} \in \operatorname{Diff}_{0} X$ is an automorphism of the complex manifold $(X, \breve{I})$. Similarly, for any $b=(\omega, H) \in \mathcal{B}$, the isotropy group $\Gamma_{b}$ is the group of automorphisms $g$ of the principal $G$-bundle $E_{H}$ such that $\check{g} \in \operatorname{Diff}_{0} X$ is a symplectomorphism of $(X, \omega)$. Hence the extended gauge group $\widetilde{\mathcal{G}_{b}}$ of $E_{H}$ on $(X, \omega)$ (defined in Section 1.3) is a subgroup of $\Gamma_{b}$, which is normal because the group of Hamiltonian symplectomorphisms is a normal subgroup of the symplectomorphism group (see eg [45, Proposition 10.2]). Note also that the fibre $B_{I}$ is a contractible space, as it is

$$
B_{I}=\mathcal{K}_{\check{I}} \times \mathcal{R}
$$

where $\mathcal{K}_{\check{I}}$ is the space of Kähler forms in $\Omega$ on the complex manifold $(X, \check{I})$. The fibre $Z_{b}$ has a gauge-theoretic description. Let $\mathcal{J}_{\omega}$ be the space of almost complex structures on $X$ compatible with $\omega$ and $\mathcal{A}_{H}$ the space of connections on $E_{H}$. Given $b=(\omega, H) \in \mathcal{B}$, define

$$
\mathcal{P}_{b} \subset \mathcal{J}_{\omega} \times \mathcal{A}_{H}
$$

as in (2.46), ie, as the space of pairs $(J, A)$ such that $J$ is integrable and $F_{A} \in$ $\Omega_{J}^{1,1}\left(\operatorname{ad} E_{H}\right)$. This subspace is clearly $\Gamma_{b}$-invariant and has an almost complex structure I given by (2.45), which is formally integrable by Proposition 2.2. Note also that for all $H \in \mathcal{R}$, each connection $A \in \mathcal{A}_{H}$ induces canonically a connection on $E^{c}$, given by $G^{c}$-equivariant maps

$$
\theta_{A}: T E^{c} \rightarrow V E^{c}, \quad \theta_{A}^{\perp}: \pi^{*} T X \rightarrow T E^{c},
$$

where $\pi: E^{c} \rightarrow X$ is the canonical projection (cf (1.27)), via the canonical isomorphism

$$
E^{c} \cong E_{H} \times{ }_{G} G^{c}
$$

of principal $G^{c}$-bundles (with $G$ acting on $G^{c}$ by left multiplication).

Lemma 3.1 The map $\pi_{\mathcal{B}}: \mathcal{C} \rightarrow \mathcal{B}$ is a " $\Gamma$-invariant almost-complex fibration", that is, its fibres $Z_{b} \subset \mathcal{Z}$ are preserved by $\mathbf{I}$ and their induced almost complex structures are exchanged by the $\Gamma$-action. Furthermore, the map

$$
\begin{aligned}
\mathbb{I}: \mathcal{P}_{b} & \longrightarrow \mathcal{Z}, \\
(J, A) & \longmapsto \mathbf{i} \theta_{A}+\theta_{A}^{\perp} \circ \pi^{*} J \circ d \pi,
\end{aligned}
$$


is a well-defined $\Gamma_{b}$-equivariant holomorphic embedding whose image is $Z_{b}$, for all $b=(\omega, H) \in \mathcal{B}$.

Proof The first assertion follows immediately from (3.62). For the second, note that (3.68) is well defined by direct computation of the Nijenhuis tensor of $\mathbb{I}(J, A)$. Using the classical construction [52] of the Chern connection $\theta_{H, I}$ of $I \in Z_{b}$ on $E_{H}$, we see that the map (3.68) is injective with image $Z_{b}$, as

$$
I=\mathbb{I}\left(\check{I}, \theta_{H, I}\right)
$$

for all $I \in Z_{b}$. Furthermore, (3.68) is clearly $\Gamma_{b}$-equivariant. Another direct computation shows now that (3.68) is a holomorphic embedding, ie, its differential is also injective and exchanges the almost complex structures on $\mathcal{P}_{b}$ and $\mathcal{Z}$.

As an immediate consequence, $Z_{b} \cong \mathcal{P}_{b}$ equipped with the restriction of $\mathbf{I}$ is a formally integrable complex manifold, by Proposition 2.2. Using Lemma 3.1, we can now transfer the constructions of Section 2.2 to the fibres

$$
Z_{b}=\mathbb{I}\left(\mathcal{P}_{b}\right),
$$

obtaining the following theorem, where the Lie groups $\Gamma_{b} \subset \Gamma$ and their normal subgroups $\mathcal{G}_{b} \subset \Gamma_{b}$, parametrised by $b \in \mathcal{B}$, are viewed as the fibres of two Lie group subbundles

$$
\tilde{\mathcal{G}}_{\mathcal{B}} \subset \Gamma_{\mathcal{B}} \subset \mathcal{B} \times \Gamma
$$

over $\mathcal{B}$. Their associated Lie algebra bundles are denoted Lie $\widetilde{\mathcal{G}}_{\mathcal{B}} \subset$ Lie $\Gamma_{\mathcal{B}} \subset \mathcal{B} \times$ Lie $\Gamma$.

Theorem 3.2 Each pair of positive real numbers $\alpha_{0}, \alpha_{1}$ determines a structure of " $\Gamma$-invariant Hamiltonian Kähler fibration" on $\pi_{\mathcal{B}}: \mathcal{C} \rightarrow \mathcal{B}$, that is, a smooth family $\omega_{\mathcal{C}}$ of Kähler forms $\omega_{b}$ on the fibres $Z_{b}$, parametrised by $b \in \mathcal{B}$, which are exchanged by the $\Gamma$-action, and a morphism

$$
\mu_{\mathcal{C}}: \mathcal{C} \longrightarrow\left(\operatorname{Lie} \widetilde{\mathcal{G}}_{\mathcal{B}}\right)^{*}
$$

of fibrations over $\mathcal{B}$, whose fibre $\mu_{b}: Z_{b} \rightarrow\left(\text { Lie } \widetilde{\mathcal{G}}_{b}\right)^{*}$ is a moment map for the $\widetilde{\mathcal{G}}_{b}-$ action on $Z_{b}$, and such that

$$
\left\langle\mu_{g \cdot b}(g \cdot I), \zeta\right\rangle=\left\langle\mu_{b}(I), \operatorname{Ad}\left(g^{-1}\right) \zeta\right\rangle
$$

for all $(b, I) \in \mathcal{C}, g \in \Gamma, \zeta \in \operatorname{Lie} \widetilde{\mathcal{G}}_{g} \cdot b$. 
Proof As in Section 2, we fix a $G$-invariant positive definite inner product on $\mathfrak{g}$. Suppose that it extends to a $G^{c}$-invariant symmetric bilinear form $(\cdot, \cdot): \mathfrak{g}^{c} \otimes \mathfrak{g}^{c} \rightarrow \mathbb{C}$ (eg, we can use $(\cdot, \cdot):=-\operatorname{tr}\left(\rho(\cdot) \circ \rho(\cdot)\right.$ ) for a faithful representation $\rho: G^{c} \rightarrow$ $\operatorname{GL}(r, \mathbb{C})$ such that $\rho(G) \subset U(r))$. This form induces another one on the adjoint bundle ad $E^{c}=E^{c} \times{ }_{G^{c}} \mathfrak{g}^{c}$, which extends to a $\mathbb{C}$-bilinear map

$$
\begin{aligned}
\Omega^{p}\left(\operatorname{ad} E^{c}\right) \times \Omega^{q}\left(\operatorname{ad} E^{c}\right) & \longrightarrow \Omega^{p+q} \otimes \mathbb{C}, \\
\left(a_{p}, a_{q}\right) & \longmapsto a_{p} \wedge a_{q},
\end{aligned}
$$

(cf (1.11)), which clearly is equivariant under the action of Aut $E^{c}$ given by pull-back. Fix $\alpha_{0}, \alpha_{1}>0$. By the results of Section 2, for each $b=(\omega, H) \in \mathcal{B}$ we have a Kähler manifold

$$
\left(Z_{b}, \mathbf{I}, \omega_{b}\right),
$$

where $\mathbf{I}$ is the restriction of (3.62) and $\omega_{b}$ corresponds to (2.43) via the isomorphism $Z_{b} \cong \mathcal{P}_{b}$ of Lemma 3.1. Furthermore, the $\widetilde{\mathcal{G}}_{b}$-action on $\left(Z_{b}, \omega_{b}\right)$ is Hamiltonian, with moment map

$$
\mu_{b}: Z_{b} \longrightarrow\left(\operatorname{Lie} \widetilde{\mathcal{G}}_{b}\right)^{*}
$$

which corresponds to the moment map in Proposition 2.1 via the isomorphism $Z_{b} \cong \mathcal{P}_{b}$ of Lemma 3.1. Using now the (Aut $E^{c}$ )-equivariance of (3.74), it is easy to see that $\omega_{b}$ and $\mu_{b}$ are the fibres of a family $\omega_{\mathcal{C}}$ defining a $\Gamma$-invariant Kähler fibration and a morphism of bundles as in (3.72), respectively.

To prove (3.73), note that the actions of Aut $E^{c}$ on the Chern connection $\theta_{H, I}$ of $H \in \mathcal{R}$ and $I \in Z_{b}$, regarded as a connection on $E^{c}$, and on its curvature $F_{H, I} \in \Omega^{2}\left(\operatorname{ad} E^{c}\right)$, satisfy

$$
g \cdot \theta_{H, I}=\theta_{g \cdot H, g \cdot I}, \quad g \cdot F_{H, I}=F_{g \cdot H, g \cdot I},
$$

for all $g \in$ Aut $E^{c}, H \in \mathcal{R}, I \in \mathcal{Z}(\operatorname{cf}[15$, Section 1.1]). Given $(b, I) \in \mathcal{C}$, we define (3.78) $S_{\alpha}(b, I):=-\alpha_{0} S_{\omega, \check{I}}-\alpha_{1} \Lambda_{\omega}^{2}\left(F_{H, I} \wedge F_{H, I}\right)+4 \alpha_{1} \Lambda_{\omega} F_{H, I} \wedge z \in C^{\infty}(X)$, where $b=(\omega, H)$ and $S_{\omega, \check{I}}$ is the scalar curvature of $(X, \check{I}, \omega)$. By the equivariance of (3.74) and the second identity in (3.77),

$$
S_{\alpha}(g b, g I)=S_{\alpha}(b, I) \circ \check{g}^{-1},
$$

for all $g \in$ Aut $E^{c}$. Combining now (3.69), (3.77) and (3.79), and making a change of variable in (2.44), we obtain (3.73), as required. 
Remark 3.3 The two fibrations (3.63) can be compared with those in Gromov [28, Section 2.C], used to see that the spaces of tamed and compatible complex structures on a symplectic vector space are contractible (cf [45, Proposition 2.51]).

\subsection{Invariant fibration by symmetric spaces}

Throughout Section 3.2, we will use the framework introduced in Section 3.1 and in particular the first part of Lemma 3.1 (however, the isomorphism $\mathcal{P}_{b} \cong Z_{b}$ of Lemma 3.1 and the families $\omega_{\mathcal{C}}$ and $\mu_{\mathcal{C}}$ of Theorem 3.2 will not be used until Section 3.3). Our task now is to construct a canonical structure of " $\Gamma$-invariant symmetric space fibration" on $\pi_{\mathcal{Z}}: \mathcal{C} \rightarrow \mathcal{Z}$, that is, symmetric space structures on the fibres $B_{I}$ that are exchanged by the $\Gamma$-action. As in Section 1.2, the Lie groups and manifolds considered here are infinite-dimensional, so one has to be careful with many standard results in finite dimensions. In particular, the Newlander-Nirenberg theorem fails in general, so we use the notion of formally integrable complex structure, as in Proposition 2.2.

Let $W$ be the space of complex structures on the real vector space underlying the Lie algebra Lie $\Gamma$ (ie, linear maps whose square is $-\mathrm{Id}$ ). Consider the tautological $\Gamma$-equivariant map

$$
\mathcal{Z} \longrightarrow W
$$

which assigns to each $I$ the endomorphism Lie $\Gamma \rightarrow$ Lie $\Gamma: y \mapsto I y$. Then, since any $I \in \mathcal{Z}$ is integrable, (3.80) satisfies the conditions

$$
Y_{I y \mid I}=\mathbf{I} Y_{y \mid I}, \quad\left[y, y^{\prime}\right]_{\Gamma}+I\left[y, I y^{\prime}\right]_{\Gamma}+I\left[I y, y^{\prime}\right]_{\Gamma}-\left[I y, I y^{\prime}\right]_{\Gamma}=0,
$$

for all $y, y^{\prime} \in \operatorname{Lie} \Gamma$ (with $[\cdot, \cdot]_{\Gamma}$ as in (3.61)), where

$$
Y_{y \mid I} \in T_{I} \mathcal{Z}
$$

is the infinitesimal action of $y \in \operatorname{Lie} \Gamma$ on $I \in \mathcal{Z}$, given by the Lie derivative $-L_{y} I$.

To construct the symmetric space fibration, we first prove that $\mathcal{Z}$ parametrises rightinvariant formally integrable complex structures on the group $\Gamma$. Given $g \in \Gamma$, define

$$
\begin{aligned}
L_{g}: & \Gamma \longrightarrow \Gamma, & R_{g}: \Gamma & \longrightarrow \Gamma, \\
h & \longmapsto g h, & h & \longmapsto h g,
\end{aligned}
$$

as the left and right multiplication by $g$, respectively. To each $I \in \mathcal{Z}$, we associate a right-invariant almost complex structure $\mathbf{I}$ on $\Gamma$, defined for $v \in T_{g} \Gamma, g \in \Gamma$ by

$$
\mathbf{I} v=\left(R_{g}\right)_{*} I\left(R_{g}\right)_{*}^{-1} v .
$$

Proposition 3.4 The almost complex structure $\mathbf{I}$ is formally integrable, for all $I \in \mathcal{Z}$. 
Proof The statement follows from the second equation in (3.81), evaluating the Nijenhuis tensor $N_{\mathbf{I}}$ of $\mathbf{I}$ on right invariant vector fields.

The next step in the construction of our symmetric space fibration relies on the following condition for all $I \in \mathcal{Z}$ such that $B_{I}$ is non-empty (this property will be proved in Proposition 3.16):

$(\star)$ There exists a well-defined isomorphism of vector bundles

$$
\text { Lie } \widetilde{\mathcal{G}}_{\mathcal{B} \mid B_{I}} \stackrel{\cong}{\rightrightarrows} T B_{I}:(b, \zeta) \longmapsto Y_{I \zeta \mid b}
$$

provided by the infinitesimal action of $I$ Lie $\widetilde{\mathcal{G}}_{b} \subset$ Lie $\Gamma$ on $B_{I}$.

In the sequel, the inverse of (3.85) is denoted

$$
\zeta_{I}: T B_{I} \longrightarrow \operatorname{Lie} \widetilde{\mathcal{G}}_{\mathcal{B} \mid B_{I}} .
$$

Given a compatible pair $(b, I) \in \mathcal{C}$, we define a space

$$
\mathcal{Y}=\mathcal{Y}_{b, I}:=\left\{g \in \Gamma \mid g \cdot b \in B_{I}\right\},
$$

a map $\pi=\pi_{b, I}: \mathcal{Y} \rightarrow B_{I}$ given by $\pi(g)=g \cdot b$ and a right $\Gamma_{b}$-action on $\mathcal{Y}$ given by right multiplication in $\Gamma$.

Proposition 3.5 For any $(b, I) \in \mathcal{C}$, the following properties hold:

(1) $\mathcal{Y}$ is principal $\Gamma_{b}$-bundle over $B_{I}$.

(2) There exists a canonical connection $\mathbb{A}$ on $\mathcal{Y}$, with horizontal lift

$$
\begin{aligned}
\theta_{\mathbb{A}}^{\perp}: \pi^{*} T B_{I} & \longrightarrow T \mathcal{Y}, \\
(g, v) & \longmapsto\left(R_{g}\right)_{*} I \zeta_{I}(v),
\end{aligned}
$$

and curvature given by

$$
F_{\mathbb{A}}\left(v_{0}, v_{1}\right)=\left(R_{g}\right)_{*}\left[\zeta_{I}\left(v_{0}\right), \zeta_{I}\left(v_{1}\right)\right]_{\Gamma},
$$

for all $g \in \mathcal{Y}$ and $v_{0}, v_{1} \in T_{g \cdot b} B_{I}$.

Proof The $\Gamma_{b}$-action on $\mathcal{Y}$ is clearly free, so leaving aside global topological questions, to prove part (1), it suffices to show that $\pi$ is surjective and induces $\mathcal{Y} / \Gamma_{b} \cong B_{I}$, that is, for all $b^{\prime} \in B_{I}$, there exists $g \in \Gamma$ such that $b^{\prime}=g \cdot b$. Since $B_{I}$ is contractible (see (3.64)), there exists a smooth curve $b_{t}$ on $B_{I}$ with $b_{0}=b, b_{1}=b^{\prime}$. Let

$$
y_{t}=I \zeta_{I}\left(\dot{b}_{t}\right) \in \operatorname{Lie} \Gamma,
$$


with $\zeta_{I}$ given by (3.86). Let $g_{t} \in \Gamma$ be the flow of $y_{t}$, defined by

$$
\dot{g}_{t} \cdot g_{t}^{-1}=y_{t},
$$

with initial condition $g_{0}=1$. Note that the flow $g_{t}$ exists for all $t$ because $y_{t}$ is $G^{c}$-invariant, so it covers a vector field $\check{y}_{t}$ on $X$, whose flow $\check{g}_{t} \in \operatorname{Diff}_{0} X$ exists for all $t$ because $X$ is compact (cf (1.26) and Remark 1.4). Now, by the Leibniz rule,

$$
\frac{d}{d t}\left(g_{t}^{-1} \cdot b_{t}\right)=g_{t}^{-1} \cdot\left(-Y_{y_{t} \mid b_{t}}+\dot{b}_{t}\right)=0,
$$

because $\zeta_{I}$ inverts the infinitesimal action of $I$ Lie $\widetilde{\mathcal{G}}_{b} \subset$ Lie $\Gamma$ on $B_{I}$ (cf [18, page 17]). Thus $g_{t}^{-1} \cdot b_{t}$ is independent of $t$, so $b^{\prime}=g_{1} \cdot b$, as required.

For (2), note that the horizontal lift of curves on $B_{I}$ to $\mathcal{Y}$ determined by the flow of (3.90) defines a canonical connection $\mathbb{A}$ on $\mathcal{Y}$. To obtain (3.88), let $b_{t}$ be a curve on $B_{I}$ with $\dot{b}_{0}=v$ and $g \in \Gamma$ such that $g \cdot b=b_{0}$. By definition, the horizontal lift $g_{t}$ of $b_{t}$ through $g$ is the flow of (3.90) with $g_{0}=g$ (recall that it exists because $y_{t}$ is $G^{c}$-invariant). Hence

$$
\theta_{\mathbb{A}}^{\perp}(g, v)=\frac{d}{d t}{ }_{\mid t=0} g_{t} g^{-1} g=\left(R_{g}\right)_{*}\left(I \zeta_{I}(v)\right) .
$$

To check (3.89), given $y \in$ Lie $\Gamma$ we denote by $\mathcal{X}_{y}$ the associated left-invariant vector field on $\Gamma$, given by

$$
\mathcal{X}_{y \mid g}:=\left(L_{g}\right)_{*} y .
$$

Since $\mathbf{I}$ is right invariant, $\left[\mathcal{X}_{y}, \mathbf{I} \cdot\right]=\mathbf{I}\left[\mathcal{X}_{y}, \cdot\right]$ for any $y \in \operatorname{Lie} \Gamma$, which implies that

$$
\left[\mathbf{I} \mathcal{X}_{y_{0}}, \mathbf{I} \mathcal{X}_{y_{1}}\right]_{\mid 1}=-\left[y_{0}, y_{1}\right]_{\Gamma}
$$

for any $y_{0}, y_{1} \in \operatorname{Lie} \Gamma$, by Proposition 3.4. Note also that

$$
\theta_{\mathbb{A}}^{\perp}(g, v)=\mathbf{I}\left(L_{g}\right)_{*}\left(\operatorname{Ad}\left(g^{-1}\right) \zeta_{I}(v)\right)=\mathbf{I} \mathcal{X}_{\operatorname{Ad}\left(g^{-1}\right) \zeta_{I}(v) \mid g}=\left(\left(R_{g}\right)_{*}\left(\mathbf{I} \mathcal{X}_{\zeta_{I}(v)}\right)_{\mid g},\right.
$$

for any $g \in \mathcal{Y}$ and $v \in T_{g b} B_{I}$. Hence given $v_{0}, v_{1} \in T_{g b} B_{I}$,

$$
\begin{aligned}
F_{\mathbb{A}}\left(v_{0}, v_{1}\right) & =-\theta_{\mathbb{A}}\left(R_{g}\right)_{*}\left[\mathbf{I} \mathcal{X}_{\zeta_{I}\left(v_{0}\right)}, \mathbf{I} \mathcal{X}_{\zeta_{I}\left(v_{1}\right)}\right]_{\mid 1} \\
& =\theta_{\mathbb{A}}\left(R_{g}\right)_{*}\left[\zeta_{I}\left(v_{0}\right), \zeta_{I}\left(v_{1}\right)\right]_{\Gamma}=\left(R_{g}\right)_{*}\left[\zeta_{I}\left(v_{0}\right), \zeta_{I}\left(v_{1}\right)\right]_{\Gamma},
\end{aligned}
$$

where the first equality follows from (1.31) and the third because

$$
\left(R_{g}\right)_{*}\left[\zeta_{I}\left(v_{0}\right), \zeta_{I}\left(v_{1}\right)\right]_{\Gamma}=\left(L_{g}\right)_{*} \operatorname{Ad}\left(g^{-1}\right)\left[\zeta_{I}\left(v_{0}\right), \zeta_{I}\left(v_{1}\right)\right]_{\Gamma}
$$

is a vertical vector field on $\mathcal{Y}$. 
Given $b, b^{\prime} \in B_{I}, b^{\prime}=g \cdot b$ for any $g$ in the fibre of $\mathcal{Y}_{b, I}$ over $b^{\prime}$, by Proposition 3.5. Then we have an isomorphism of principal bundles

$$
\begin{aligned}
\mathcal{Y}_{b, I} & \cong \mathcal{Y}_{b^{\prime}, I}, \\
g^{\prime} & \longmapsto g^{\prime} g^{-1},
\end{aligned}
$$

with corresponding isomorphism $\Gamma_{b} \stackrel{\cong}{\rightrightarrows} \Gamma_{b^{\prime}}: g^{\prime} \longmapsto \operatorname{Ad}(g) g^{\prime}$ between their structure groups. It follows from the definition of the canonical connection in terms of (3.90), or from (3.88), that this isomorphism exchanges the canonical connections on these principal bundles.

We are now in a position to construct the promised canonical structure of " $\Gamma$-invariant symmetric space fibration" on $\pi_{\mathcal{Z}}: \mathcal{C} \rightarrow \mathcal{Z}$. Observe first that the connection (3.88) induces a canonical affine connection

$$
\nabla: \Omega_{B}^{0}(T B) \longrightarrow \Omega_{B}^{1}(T B)
$$

on $B_{I}$, obtained using the canonical isomorphism

$$
T B_{I} \cong \mathcal{Y} \times_{\Gamma_{b}} \text { Lie } \widetilde{\mathcal{G}}_{b} \subset \text { ad } \mathcal{Y},
$$

which follows from the canonical isomorphism $T B_{I} \cong \pi^{*} T B_{I} / \Gamma_{b}$ and the $\Gamma_{b}-$ equivariant isomorphism of vector bundles

$$
\begin{aligned}
\mathcal{Y} \times \operatorname{Lie} \tilde{\mathcal{G}}_{b} & \cong \pi^{*} T B_{I}, \\
(g, \zeta) & \longmapsto\left(g, Y_{I \operatorname{Ad}(g) \zeta \mid g \cdot b}\right) .
\end{aligned}
$$

Note also that the parallel transport $\tau_{t}(v)$ of a tangent vector $v \in T_{b_{0}} B_{I}$ along a curve $b_{t}$ on $B_{I}$, and hence the affine connection $\nabla$, do not depend on the choice of the base point $b \in \mathcal{B}_{I}$ used implicitly in the right-hand side of (3.95). In fact, it is given by the curve on $T B_{I}$ defined as

$$
\tau_{t}(v)=Y_{I \zeta_{t} \mid b_{t}}, \quad \text { where } \zeta_{t}:=\operatorname{Ad}\left(g_{t}\right) \zeta_{I}(v) .
$$

Here, $g_{t}$ is the flow of (3.90) with $g_{0}=1$. This follows from (3.95), (3.96) and standard properties about horizontal lifts (Kobayashi and Nomizu [35, page 114]).

Note that the canonical connections (3.88) and (3.94) are constructed exactly as for any finite-dimensional symmetric space (cf eg [36, Ch. XI, Section 3]) and that they are exchanged by the $\Gamma$-actions. In fact, our next result shows that $\left(B_{I}, \nabla\right)$ is a symmetric space, in a similar sense to [18, Section 4, Proposition 2].

Theorem 3.6 Let $I \in \mathcal{Z}$ be such that $B_{I}$ is non-empty. Then $B_{I}$ is a symmetric space, ie, it has a torsion-free affine connection $\nabla$, with holonomy group contained in 
$\widetilde{\mathcal{G}}_{b}$ and covariantly constant curvature $R_{\nabla}$, given by

$$
\zeta_{I}\left(R_{\nabla}\left(v_{0}, v_{1}\right) v_{2}\right)=\left[\left[\zeta_{I}\left(v_{0}\right), \zeta_{I}\left(v_{1}\right)\right]_{\Gamma}, \zeta_{I}\left(v_{2}\right)\right]_{\Gamma},
$$

for any $b \in B_{I}$ and $v_{0}, v_{1}, v_{2} \in T_{b} B_{I}$.

Proof To prove this, we relate the torsion $T_{\nabla}$ of $\nabla$ with the Nijenhuis tensor $N_{\mathrm{I}}$ of $(\Gamma, \mathbf{I})$ and its curvature $R_{\nabla}$ with the curvature $F_{\mathbb{A}}$ of $\mathbb{A}$.

Let $V_{1}$ and $V_{2}$ be two vector fields on $B_{I}$. Then

$$
T_{\nabla}\left(V_{1}, V_{2}\right):=\nabla_{V_{1}} V_{2}-\nabla_{V_{2}} V_{2}-\left[V_{1}, V_{2}\right] .
$$

Consider the principal $\Gamma_{b}$-bundle $\pi: \mathcal{Y} \rightarrow B_{I}$ associated to a fixed $b \in B_{I}$. By (3.95), $T B_{I}$ is a subbundle of ad $\mathcal{Y}$, so $V_{j}$ induces a $\Gamma_{b}$-invariant vertical vector fields $\widehat{V}_{j}$ on $\mathcal{Y}$, given by

$$
\widehat{V}_{j}(g)=\left(R_{g}\right)_{*} \zeta_{I}\left(V_{j}(g b)\right),
$$

for $g \in \mathcal{Y}, j=0,1$. We claim that

$$
T_{\nabla}\left(V_{1}, V_{2}\right)=-d \pi\left(N_{\mathbf{I}}\left(\widehat{V}_{1}, \widehat{V}_{2}\right)\right),
$$

and so $T_{\nabla}=0$ by Proposition 3.4. To see this, note first that

$$
\mathbf{I} \widehat{V}_{j}=\theta_{\mathbb{A}}^{\perp} V_{j} \quad \text { and } \quad F_{\mathbb{A}}\left(V_{1}, V_{2}\right)=-\left[\widehat{V}_{1}, \widehat{V}_{2}\right],
$$

by (3.88) and (3.89). Moreover, by the construction of $\nabla$ and the definition of the covariant derivative $d_{\mathbb{A}}$ induced by $\mathbb{A}$ on ad $\mathcal{Y}$ (see (1.31)),

$$
\widehat{\nabla V}_{j}=d_{\mathbb{A}} \widehat{V}_{j}:=\left[\theta_{\mathbb{A}}^{\perp}(\cdot), \widehat{V}_{j}\right]=\left[\mathbf{I}(\cdot), \widehat{V}_{j}\right]
$$

It follows then that

$$
\begin{aligned}
N_{\mathbf{I}}\left(\widehat{V}_{1}, \widehat{V}_{2}\right): & =\left[\mathbf{I} \widehat{V}_{1}, \mathbf{I} \widehat{V}_{2}\right]-\mathbf{I}\left[\mathbf{I} \widehat{V}_{1}, \widehat{V}_{2}\right]-\mathbf{I}\left[\widehat{V}_{1}, \mathbf{I} \widehat{V}_{2}\right]-\left[\widehat{V}_{1}, \widehat{V}_{2}\right] \\
& =\left[\theta_{\mathbb{A}}^{\perp} V_{1}, \theta_{\mathbb{A}}^{\perp} V_{2}\right]-\mathbf{I}{\widehat{\nabla_{V_{1}} V_{2}}}+\mathbf{I} \widehat{\nabla}_{V_{2} V_{1}}+F_{\mathbb{A}}\left(V_{1}, V_{2}\right) \\
& =\theta_{\mathbb{A}}^{\perp}\left(\left[V_{1}, V_{2}\right]-\nabla_{V_{1}} V_{2}+\nabla_{V_{2}} V_{1}\right) \\
& =-\theta_{\mathbb{A}}^{\perp} T_{\nabla}\left(V_{1}, V_{2}\right),
\end{aligned}
$$

and so (3.99) holds.

Since the curvature $R_{\nabla}$ is induced by $F_{\mathbb{A}}$ via the adjoint representation, it follows from (3.89), (3.95) and the fact that Lie $\widetilde{\mathcal{G}}_{b} \subset$ Lie $\Gamma$ is a Lie subalgebra, that

$$
R_{\nabla}\left(v_{0}, v_{1}\right) v_{2}=Y_{I\left[\left[\zeta_{I}\left(v_{0}\right), \zeta_{I}\left(v_{1}\right)\right]_{\Gamma}, \zeta_{I}\left(v_{2}\right)\right]_{\Gamma} \mid b},
$$

for $v_{0}, v_{1}, v_{2} \in T_{b} B_{I}$, which implies (3.98), by condition ( $\star$ ). Hence, since the group $\widetilde{\mathcal{G}}_{b}$ is normal in $\Gamma_{b}$ and $B_{I}$ is contractible, it follows from (3.100) that the holonomy 
group of $\nabla$ is contained in $\widetilde{\mathcal{G}}_{b}$ (see [35, Theorem 8.1]). Using (3.100) and the formula (3.97) for the parallel transport $\tau_{t}$ of a curve on $B_{I}$, it is now straightforward that $\tau_{t}^{*} R_{\nabla}=R_{\nabla}$, so $\nabla R_{\nabla}=0$.

Remark 3.7 When $H^{1}(X, \mathbb{R})=0$, so Lie $\widetilde{\mathcal{G}}_{b}=$ Lie $\Gamma_{b}$, it follows from Proposition 3.5(1) that the bundle $\mathcal{Y}$, endowed with the restriction of the formally integrable almost complex structure of Proposition 3.4, is an infinitesimal complexification of $\Gamma_{b}$ in the sense of Donaldson [18, Section 4]. If in addition $\Gamma_{I}$ is trivial, then there is an alternative proof of Theorem 3.6 that does not use Proposition 3.4. In this case, the almost complex structure on $\mathcal{Y}_{b, I}$ can be defined as the pull-back of the formally integrable almost complex structure on $Z_{b}$ by the holomorphic map

$$
\begin{aligned}
\mathcal{Y}_{b, I} & \longrightarrow Z_{b}, \\
g & \longmapsto g^{-1} I .
\end{aligned}
$$

\subsection{The uniqueness and existence problem for the coupled equations}

We apply now the framework of Section 3.1, 3.2 to construct obstructions to the existence of solutions to the coupled equations (2.47).

Fix coupling constants $\alpha_{0}, \alpha_{1}>0$. It follows from Proposition 2.4, Lemma 3.1 and the construction of $\mu_{b}$ in Theorem 3.2 for each $b=(\omega, H) \in \mathcal{B}$, that the existence of a solution $(J, A) \in \mathcal{P}_{b}$ of the coupled equations (2.47) (for the symplectic manifold $(X, \omega)$ and the principal $G$-bundle $\left.E_{H}\right)$ is equivalent to the condition $\mu_{b}(I)=0$ for some $I \in Z_{b}$. By the equivariance (3.73) of $\mu_{\mathcal{C}}$, this is equivalent to the condition

$$
\pi_{\mathcal{Z}}^{-1}(\Gamma \cdot I) \cap \mu_{\mathcal{C}}^{-1}(0) \neq \varnothing,
$$

where $\Gamma \cdot I \subset \mathcal{Z}$ is the orbit of $I$. Given such an orbit, in Section 3.3 we construct a complex character $\mathcal{F}_{I}$ of the complex Lie algebra Lie $\Gamma_{I}$, which vanishes when (3.102) is satisfied, and an "integral of the moment map" $\mathcal{M}_{I}: B_{I} \rightarrow \mathbb{R}$, which is bounded from below when (3.102) is satisfied, provided that the symmetric space $B_{I}$ is geodesically convex. Furthermore, we motivate a definition of "geodesic stability" of the orbit $\Gamma \cdot I$ and conjecture a link with (3.102) when $\Gamma_{I}$ is finite.

We first reformulate condition (3.102) in terms of a $\Gamma$-invariant family $\sigma$ of 1 -forms $\sigma_{I}$ on the fibres $B_{I}$ of $\pi_{\mathcal{Z}}: \mathcal{C} \rightarrow \mathcal{Z}$, defined by the formula

$$
\sigma_{I}(v):=-\left\langle\mu_{b}(I), \zeta_{I}(v)\right\rangle,
$$

for all $(b, I) \in \mathcal{C}, v \in T_{b} B_{I}$, with $\zeta_{I}$ defined as in (3.86). Here, the $\Gamma$-invariance of $\sigma$ means

$$
\sigma_{g \cdot I}(g v)=\sigma_{I}(v)
$$


for all $(b, I) \in \mathcal{C}, v \in T_{b} B_{I}, g \in \Gamma$. Note that (3.104) follows from (3.73) and the fact that

$$
\operatorname{Ad}(g) \zeta_{I}(v)=\zeta_{g I}(v)
$$

for all $g \in \Gamma$, which is immediate from the definition of $\zeta_{I}$. Observe also that

$$
\pi_{\mathcal{Z}}^{-1}(\Gamma \cdot I) \cap \mu_{\mathcal{C}}^{-1}(0) \neq \varnothing \Longleftrightarrow \sigma_{I} \in \Omega^{1}\left(B_{I}\right) \text { has a zero. }
$$

Now, since $B_{I}$ is contractible (see (3.64)), it suffices to study $\sigma_{I}$ along curves on $B_{I}$. Let $V_{t}$ be a vector field on $B_{I}$ along a curve $b_{t}$ on $B_{I}$, ie, a curve on $T B_{I}$ with $V_{t} \in T_{b_{t}} B_{I}$ for all $t$. We use the standard notation $\nabla_{\dot{b}_{t}} V_{t}$ for the covariant derivative of $V_{t}$ in the direction of $\dot{b}_{t}$ on the symmetric space $\left(B_{I}, \nabla\right)$ (see (3.94) and Theorem 3.6).

\section{Proposition 3.8}

(1) $\frac{d}{d t} \sigma_{I}\left(V_{t}\right)=\omega_{b_{t}}\left(Y_{\zeta_{I}\left(V_{t}\right) \mid I}, \mathbf{I} Y_{\zeta_{I}\left(\dot{b}_{t}\right) \mid I}\right)+\sigma_{I}\left(\nabla_{\dot{b}_{t}} V_{t}\right)$

(2) $\sigma_{I}$ is closed.

Proof To prove (1), let $g_{t}$ the horizontal lift of $b_{t}$ to $\mathcal{Y}_{b_{0}, I}$ prescribed by the connection (3.88), with $g_{0}=1$. Then $b_{t}=g_{t} \cdot b$ (see Proposition 3.5), so (3.73) implies

$$
\sigma_{I}\left(V_{t}\right)=-\left\langle\mu_{b}\left(I_{t}\right), \zeta_{t}\right\rangle
$$

where $I_{t}:=g_{t}^{-1} \cdot I$ and $\zeta_{t}:=\operatorname{Ad}\left(g_{t}\right)^{-1} \zeta_{I}\left(V_{t}\right)$. Using (3.81), we obtain

$$
\dot{I}_{t}=-g_{t}^{-1} \dot{g}_{t} g_{t}^{-1} I=-g_{t}^{-1} Y_{I \zeta_{I}\left(\dot{b}_{t}\right) \mid I}=-g_{t}^{-1} \mathbf{I} Y_{\zeta_{I}\left(\dot{b}_{t}\right) \mid I}
$$

so using formula (3.97) for the parallel transport $\tau_{t, s}: T_{b_{t}} B_{I} \rightarrow T_{b_{s}} B_{I}$ and the definition of covariant derivative (see eg [35, page 114]):

$$
\begin{aligned}
\nabla_{\dot{b}_{t}} V_{t} & :=\frac{d}{d s \mid s=t} \tau_{t, s}^{-1}\left(V_{t}\right)=\frac{d}{d s}{ }_{\mid s=t} Y_{I \operatorname{Ad}\left(g_{s} g_{t}^{-1}\right)^{-1} \zeta_{I}\left(V_{t}\right) \mid b_{t}} \\
& =Y_{I \operatorname{Ad}\left(g_{t}\right) \dot{\zeta}_{t} \mid b_{t}}=g_{t} Y_{I_{t} \dot{\zeta}_{t} \mid b}
\end{aligned}
$$

Formula (1) follows now from this equation and the $\Gamma$-invariance of $\sigma_{I}$, as they imply $\sigma_{I_{t}}\left(Y_{I_{t} \dot{\zeta}_{t} \mid b}\right)=\sigma_{I}\left(\nabla_{\dot{b}_{t}} V_{t}\right)$, which combined with (3.106) implies

$$
\begin{aligned}
\frac{d}{d t} \sigma_{I}\left(V_{t}\right) & \left.=-\left\langle d \mu_{b}\left(\dot{I}_{t}\right), \zeta_{t}\right)\right\rangle-\left\langle\mu_{b}\left(I_{t}\right), \dot{\zeta}_{t}\right\rangle \\
& =\omega_{b}\left(g_{t}^{-1} Y_{\zeta_{I}\left(V_{t}\right) \mid I}, g_{t}^{-1} \mathbf{I} Y_{\zeta_{I}\left(\dot{b}_{t}\right) \mid I}\right)+\sigma_{I_{t}}\left(Y_{I_{t} \dot{\zeta}_{t} \mid b}\right) \\
& =\omega_{b_{t}}\left(Y_{\zeta_{I}\left(V_{t}\right) \mid I}, \mathbf{I} Y_{\zeta_{I}\left(\dot{b}_{t}\right) \mid I}\right)+\sigma_{I}\left(\nabla_{\dot{b}_{t}} V_{t}\right),
\end{aligned}
$$

since $\mu_{b}$ is a moment map and $\omega_{\mathcal{C}}$ is $\Gamma$-invariant. 
To prove (2), let $V_{1}$ and $V_{2}$ be two vector fields on $B_{I}$. Then

$$
d \sigma_{I}\left(V_{1}, V_{2}\right)=V_{1}\left(\sigma_{I}\left(V_{2}\right)\right)-V_{2}\left(\sigma_{I}\left(V_{1}\right)\right)-\sigma_{I}\left(\left[V_{1}, V_{2}\right]\right),
$$

so, using (1) and the fact that $\omega_{\mathcal{B}}(\cdot, \mathbf{I} \cdot)$ is a family of symmetric bilinear forms, we see that

$$
d \sigma_{I}\left(V_{1}, V_{2}\right)=\sigma_{I}\left(T_{\nabla}\left(V_{1}, V_{2}\right)\right),
$$

which vanishes because $\nabla$ is torsion-free, by Theorem 3.6.

To define our first obstruction to (3.102), note that Lie $\Gamma_{I}$ is a complex Lie algebra for all $I \in \mathcal{Z}$, by (3.81) and the equivariance of (3.80). Given $I \in \mathcal{Z}$ and $b \in B_{I}$, combining the 1 -form $\sigma_{I}$ and (3.80), we obtain a $\mathbb{C}$-linear map

$$
\begin{aligned}
\mathcal{F}_{I}: \operatorname{Lie} \Gamma_{I} & \longrightarrow \mathbb{C}, \\
\zeta & \longmapsto\left\langle\mathcal{F}_{I}, \zeta\right\rangle:=\mathbf{i} \sigma_{I}\left(Y_{\zeta \mid b}\right)+\sigma_{I}\left(Y_{I \zeta \mid b}\right) .
\end{aligned}
$$

By the $\Gamma$-invariance of $\sigma$ (see (3.104)), this map is also $\Gamma$-invariant, ie,

$$
\left\langle\mathcal{F}_{g \cdot I}, \operatorname{Ad}(g) \zeta\right\rangle=\left\langle\mathcal{F}_{I}, \zeta\right\rangle
$$

for all $\zeta \in \operatorname{Lie} \Gamma_{I}, g \in \Gamma$.

Theorem 3.9 The map (3.108) is independent of $b \in B_{I}$. It defines a character

$$
\mathcal{F}_{I}: \text { Lie } \Gamma_{I} \longrightarrow \mathbb{C}
$$

of Lie $\Gamma_{I}$ that vanishes if $\sigma_{I}$ has a zero.

Proof The proof essentially follows a previous one by Bourguignon [7]. For the first part, it is enough to prove that $\sigma_{I}\left(Y_{\zeta}\right)$ is a constant function on $B_{I}$, for all $\zeta \in \operatorname{Lie} \Gamma_{I}$. Now, $\sigma_{I} \in \Omega^{1}\left(B_{I}\right)$ is closed (by Proposition 3.8) and $\Gamma_{I}$-invariant (since $\sigma$ is $\Gamma$-invariant), so

$$
\left.d\left(\sigma_{I}\left(Y_{\zeta}\right)\right)=-Y_{\zeta}\right\lrcorner d \sigma_{I}+L_{Y_{\zeta}} \sigma_{I}=0,
$$

and hence $\sigma_{I}\left(Y_{\zeta}\right)$ is constant, because $B_{I}$ is contractible. The second part follows because $\mathcal{F}_{I}$ is $\mathbb{C}$-linear and $\Gamma_{I}$-invariant, by (3.109).

To obtain the second obstruction, note that, by Proposition 3.8 and the contractibility of $B_{I}, \sigma_{I}$ is exact and so there exists a functional

$$
\mathcal{M}_{I}: B_{I} \times B_{I} \rightarrow \mathbb{R}
$$


such that $d \mathcal{M}_{I}(\cdot, b)=\sigma_{I}$ and $\mathcal{M}_{I}(b, b)=0$ for all $b \in B_{I}$. Along a curve $b_{t}$ on $B_{I}$,

$$
\mathcal{M}_{I}\left(b_{t}, b\right)=\mathcal{M}_{I}\left(b_{0}, b\right)+\int_{0}^{t} \sigma_{I}\left(\dot{b}_{s}\right) d s
$$

Moreover, the $\Gamma$-invariance of $\sigma$ implies that

$$
\mathcal{M}_{I}\left(g b^{\prime}, b\right)=\mathcal{M}_{g^{-1} I}\left(b^{\prime}, b\right)+\mathcal{M}_{I}\left(b^{\prime}, b\right),
$$

for all $g \in \Gamma$ such that $g b^{\prime} \in B_{I}$ (ie, $g \in \mathcal{Y}_{b^{\prime}, I}$ ).

Proposition 3.10 The functional $\mathcal{M}_{I}(\cdot, b): B_{I} \rightarrow \mathbb{R}$ is convex along geodesics on $\left(B_{I}, \nabla\right)$. If $B_{I}$ is geodesically convex and $\sigma_{I}$ has a zero, then $\mathcal{M}_{I}(\cdot, b)$ is bounded from below, for all $b \in B_{I}$.

Proof The first part follows because (3.111) and Proposition 3.8(1) imply

$$
\frac{d^{2}}{d t^{2}} \mathcal{M}_{I}\left(b_{t}, b\right)=\frac{d}{d t} \sigma_{I}\left(\dot{b}_{t}\right)=\left\|Y_{\zeta_{I}\left(\dot{b}_{t}\right) \mid I}\right\|^{2} \geq 0,
$$

for any geodesic $b_{t}$ on $\left(B_{I}, \nabla\right)$, where $\|\cdot\|$ is the $L^{2}$-norm with respect to the metric on $Z_{b_{t}}$.

For the second part, suppose $b^{\prime} \in B_{I}$ is a zero of $\sigma_{I}$. We can suppose $b^{\prime}=b$, because using (3.111) along a curve joining $b$ and $b^{\prime}$, we see that

$$
\mathcal{M}_{I}\left(\cdot, b^{\prime}\right)=\mathcal{M}_{I}\left(b, b^{\prime}\right)+\mathcal{M}_{I}(\cdot, b) .
$$

Now, given $b^{\prime \prime} \in B_{I}$, by hypothesis there exists a geodesic $b_{t}$ with $b_{0}=b$ and $b_{1}=b^{\prime \prime}$. Hence

$$
\mathcal{M}_{I}\left(b^{\prime \prime}, b\right)=\int_{0}^{1} \int_{0}^{t}\left\|Y_{\zeta_{I}\left(\dot{b}_{s}\right) \mid I}\right\|^{2} d s \wedge d t \geq 0,
$$

and so $\mathcal{M}_{I}(\cdot, b)$ is bounded from below by $0 \in \mathbb{R}$.

Corollary 3.11 If $B_{I}$ is geodesically convex, then $\sigma_{I}$ has at most one zero on $B_{I}$ modulo the action of $\Gamma_{I}$.

Proof Given zeros $b, b^{\prime} \in B_{I}$ of $\sigma_{I}$, let $b_{t}$ a geodesic joining them. Then

$$
\left\|Y_{\zeta_{I}\left(\dot{b}_{t}\right) \mid I}\right\|^{2}=0
$$

for all $t$, because (3.113) implies that

$$
\begin{aligned}
& \mathbb{R} \longrightarrow \mathbb{R}, \\
& t \longmapsto \sigma\left(\dot{b}_{t}\right)
\end{aligned}
$$


is an increasing function which vanishes for $t=0$ and $t=1$. Hence the flow $g_{t}$ of $I \zeta_{I}\left(\dot{b}_{t}\right)$ lies in $\Gamma_{I}$ for all $t$ and $g_{t} b=b_{t}$. In particular, $g_{1} b=b^{\prime}$.

Remark 3.12 Proposition 3.8 and Theorem 3.9 hold even when $\alpha_{0}, \alpha_{1}$ are not positive (their proofs depend only on the condition that $\omega_{b}$ is of type $(1,1)$ with respect to I). In Section 4, we will use these facts about $\sigma_{I}$ and $\mathcal{F}_{I}$ for arbitrary $\alpha_{0}, \alpha_{1}$. However, Proposition 3.10, Corollary 3.11 and the remainder of Section 3.3 depend on the assumption that $\alpha_{0}, \alpha_{1}$ are positive, although Proposition 3.14 also holds in the degenerate case $\alpha_{0} \alpha_{1}=0$.

If $Z_{b}$ and $\Gamma_{b}$ are finite-dimensional manifolds and $\widetilde{\mathcal{G}}_{b}=\Gamma_{b}$ is compact, there is a wellknown numerical condition, called the Hilbert-Mumford criterion, which characterises (3.102) (see the example at the end of Section 3.3). In this case, the principal bundle $\mathcal{Y}$ of Proposition 3.5 is the complexification of $\Gamma_{b}$ (by the observations about infinitesimal complexifications at the end of Section 3.2, as formally integrable almost complex structures are integrable in finite dimensions), and the criterion is formulated in terms of 1-parameter subgroups of $\mathcal{Y}$. In the generality of Section 3, the Lie group $\Gamma_{b}$ may have no complexification, but the geodesics of the symmetric space $\left(B_{I}, \nabla\right)$ are a substitute for the 1-parameter subgroups, and we have the following generalization of this condition (cf $[11 ; 18$, Section 8]).

Definition 3.13 A point $I \in \mathcal{Z}$ is geodesically semistable if

$$
\lim _{t \rightarrow \infty} \sigma_{I}\left(\dot{b}_{t}\right) \geq 0
$$

for any infinite geodesic ray $b_{t}, t \in\left[0, \infty\left[\right.\right.$, in $\left(B_{I}, \nabla\right)$. It is geodesically stable if the inequality (3.115) is strict whenever $b_{t}$ is non-constant.

Observe that the limit (3.115) always exists, because (3.114) is an increasing function for geodesic rays, by (3.113). Note also that the geodesic stability and semistability conditions only depend on the $\Gamma$-orbit of $I \in \mathcal{Z}$, because $\sigma$ is $\Gamma$-invariant and the connections on the fibres of $\pi_{\mathcal{Z}}$ are exchanged by the $\Gamma$-action and hence so are their geodesic rays.

In the finite-dimensional case, by the Kempf-Ness Theorem [33], an orbit $\Gamma \cdot I \in \mathcal{Z}$ is geodesically stable if and only if (3.102) holds and $\Gamma_{I}$ is finite (see the example at the end of Section 3.3). The following result provides some evidence that a sensible question is whether this equivalence also holds in the generality of Section 3, at least when $B_{I}$ is geodesically convex. 
Proposition 3.14 Let $(b, I) \in \mathcal{C}$. Then:

(1) If $\Gamma_{b, I}:=\Gamma_{b} \cap \Gamma_{I}$ is not finite, then $\Gamma \cdot I$ is not geodesically stable.

(2) Suppose that $B_{I}$ is geodesically convex. If (3.102) is satisfied, then $\Gamma \cdot I$ is geodesically semistable.

Proof For part (1), let $\zeta \in \operatorname{Lie} \Gamma_{I}$ be non-zero. Let $g_{t}$ the flow of $I \zeta$. Then $b_{t}=g_{t} b$ is an infinite geodesic ray starting at $b$, because:

$$
\dot{b}_{t}=g_{t} Y_{I \zeta \mid b}=Y_{\operatorname{Ad}\left(g_{t}\right) I \zeta \mid b_{t}}=Y_{I \operatorname{Ad}\left(g_{t}\right) \xi \mid b_{t}}=\tau_{t} \dot{b}_{0} .
$$

Furthermore, if $\zeta \in \operatorname{Lie} \Gamma_{b, I}$, then $\dot{b}_{0}=Y_{I \zeta \mid b} \neq 0$, by (3.85), so $b_{t}$ is non-constant. Then

$$
\sigma_{I}\left(\dot{b}_{t}\right)=\sigma_{I}\left(g_{t} \dot{b}_{0}\right)=\sigma_{g_{t}^{-1}}\left(\dot{b}_{0}\right)=\sigma_{I}\left(\dot{b}_{0}\right)
$$

and so

$$
\lim _{t \rightarrow \infty} \sigma_{I}\left(\dot{b}_{t}\right)=\sigma_{I}\left(\dot{b}_{0}\right)=\sigma_{I}\left(Y_{I \zeta \mid b}\right)=\left\langle\mathcal{F}_{I}, \zeta\right\rangle
$$

There are three possibilities. If $\left\langle\mathcal{F}_{I}, \zeta\right\rangle<0$, then part (1) is obvious. The case $\left\langle\mathcal{F}_{I}, \zeta\right\rangle>0$ reduces to the previous one by taking the non-trivial geodesic corresponding to $-I \zeta$. Finally, if $\left\langle\mathcal{F}_{I}, \zeta\right\rangle=0$, since $b_{t}$ is non-trivial, then by definition $I$ is not geodesically stable.

For part (2), suppose that $B_{I}$ is geodesically convex and $\Gamma \cdot I$ is not geodesically semistable. Then there exists an infinite geodesic ray $b_{t}$ such that

$$
C:=\lim _{t \rightarrow \infty} \sigma_{I}\left(\dot{b}_{t}\right)<0,
$$

where $\sigma_{I}\left(\dot{\sigma}_{t}\right) \leq C$ for all $t$, as (3.114) is an increasing function, so $\mathcal{M}_{I}\left(b_{t}, b_{0}\right) \leq C t$, by (3.111). Therefore $\mathcal{M}_{I}\left(\cdot, b_{0}\right)$ is not bounded from below, so (3.102) cannot be satisfied, by Proposition 3.10.

We would like to point out that the framework developed in Section 3.2, 3.3 is rather general, as it relies only on formal properties of the double fibration (3.63), and may be applied to other situations (in particular, to equations with a further coupling with Higgs fields). The basic ingredients are a real Lie group $\Gamma$, a $\Gamma$-equivariant double fibration (3.63), where $(\mathcal{Z}, \mathbf{I})$ is an almost complex manifold, and a $\Gamma$-equivariant map (3.80) satisfying (3.81). It is crucial that $\pi_{\mathcal{Z}}$ satisfies condition $(\star)$ of Section 3.2, all its fibres are contractible and $\pi_{\mathcal{B}}$ satisfies the properties of Theorem 3.2 for a fibration of normal subgroups as in (3.71) (note that the formal integrability of the almost complex structures on the fibres of $\pi_{\mathcal{B}}$ was never used). 
To see how this general framework works, we conclude Section 3.3 by explaining how it applies to the standard theory of finite-dimensional Kähler quotients (as presented eg in [49, Section 5]) and its relation with geometric invariant theory (GIT). Suppose that $\mathcal{Z}$ is a finite-dimensional Kähler manifold with a left action of a complex reductive Lie group $G^{c}$ preserving its complex structure. Suppose also that this action restricts to a Hamiltonian action of a maximal compact subgroup $G \subset G^{c}$, with $G$-equivariant moment map

$$
\mu: \mathcal{Z} \longrightarrow \mathfrak{g}^{*},
$$

where $\mathfrak{g}$ is the Lie algebra of $G$. To compare with Section 3.2, 3.3, we define:

- $\Gamma$ is the real Lie group underlying $G^{c}$.

- $\mathcal{B}=G^{c} / G$ is the orbit space for the action by right multiplication of $G$ on $G^{c}$.

- The map $\mathcal{Z} \rightarrow W$ of (3.80) is the constant map given by the complex structure on the Lie algebra $\mathfrak{g}^{c}$ of $G^{c}$.

- $\mathcal{C}=\mathcal{B} \times \mathcal{Z}$ and $\widetilde{\mathcal{G}}_{\mathcal{B}}=\Gamma_{\mathcal{B}}$.

Then the isotropy group of any $G$-orbit $b=[g]:=g G \in \mathcal{B}$ is

$$
\widetilde{\mathcal{G}}_{b}=\Gamma_{b}=\operatorname{Ad}(g) G
$$

and the fibre of the morphism (3.85) over a point $b=[g]$ is

$$
\begin{aligned}
\operatorname{Ad}(g) \mathfrak{g} & \cong \\
\zeta & T_{g} G^{c} / T_{g}(g G), \\
\zeta & \left.\longmapsto\left(R_{g}\right)_{*}(\mathbf{i} \zeta)\right] .
\end{aligned}
$$

Therefore (3.85) is an isomorphism and condition $(\star)$ of Section 3.2 is satisfied, and hence so are the conclusions of Section 3.2 and Section 3.3. In this finite-dimensional case, the construction of the connections (3.88) and (3.94) reduce to the classical constructions of the canonical connections on finite-dimensional symmetric spaces (see eg [36, Ch. XI, Section 3]). Hence, by [36, Ch. XI, Theorem 3.2(3)], the infinite geodesic rays on $G^{c} / G$ starting at $[g]$ are the curves

$$
\begin{aligned}
{[0, \infty[} & \longrightarrow G^{c} / G, \\
t & \longmapsto\left[e^{t \mathbf{i} \zeta} g\right],
\end{aligned}
$$

with $\zeta \in \operatorname{Ad}(g) \mathfrak{g}$. Note that the canonical projection

$$
\pi_{\mathcal{B}}: G^{c} / G \times \mathcal{Z} \longrightarrow G^{c} / G
$$

is a "trivial" $G^{c}$-invariant complex fibration. However, since $G^{c}$ does not necessarily preserve $\omega_{\mathcal{Z}}$, to view $\pi_{\mathcal{B}}$ as a $G^{c}$-invariant Hamiltonian fibration, we endow this map with the non-trivial family $\omega_{\mathcal{C}}$ of symplectic $2-$ forms $\omega_{b}:=g_{*} \omega_{\mathcal{Z}}$ on the fibres 
$Z_{b}=\mathcal{Z}$, for $b=[g] \in G^{c} / G$. Indeed, the isotropy group $\operatorname{Ad}(g) G$ preserves $\omega_{b}$ and has moment map given by

$$
\left\langle\mu_{b}(I), \zeta\right\rangle:=\left\langle\mu\left(g^{-1} I\right), \operatorname{Ad}\left(g^{-1}\right) \zeta\right\rangle,
$$

for $b=[g] \in G^{c} / G$, and (3.118) defines the morphism (3.72) of fibrations over $G^{c} / G$. Using the isomorphism (3.116), we obtain the formula

$$
\left\langle\mathcal{F}_{I}, \zeta\right\rangle=-\left\langle\mu(I), \zeta_{0}\right\rangle-\mathbf{i}\left\langle\mu(I), \zeta_{1}\right\rangle
$$

for all $I \in \mathcal{Z}, \zeta=\zeta_{0}+\mathbf{i} \zeta_{1} \in \mathfrak{g}_{I}^{c}$, where $\zeta_{0}, \zeta_{1} \in \mathfrak{g}^{c}$. Hence Theorem 3.9 reduces to Wang [59, Proposition 6 and Corollary 8].

Suppose now that $\mathcal{Z}$ is a $G^{c}$-linearised projective manifold, ie, there is a $G^{c}$-equivariant closed embedding $\mathcal{Z} \subset \mathbb{C} \mathbb{P}^{N}$ and $\omega_{\mathcal{Z}}$ is the restriction of the Fubini-Study Kähler form. Then geodesic stability/semistability coincide with GIT stability/semistability, by the Hilbert-Mumford criterion. This essentially follows because any 1-parameter subgroup

$$
\lambda: \mathbb{C}^{*} \rightarrow G^{c}
$$

restricts to a group homomorphism $\lambda: S^{1} \rightarrow \operatorname{Ad}(g) G$ for some $g \in G^{c}$, which induces an infinite geodesic ray (3.117) starting at $[g]$ and because the Hilbert-Mumford weight for $\lambda$ at a point $I \in \mathcal{Z}$ is precisely the left-hand side of (3.115). Furthermore, the functional (3.110) is the Kempf-Ness functional [33], which provides the key tool to prove the Kempf-Ness theorem relating the symplectic and GIT quotients:

$$
\mu^{-1}(0) / G \cong \mathcal{Z} / / G^{c}
$$

Finally, we should remark that this theorem has been extended to non-projective manifolds (see eg [49, Section 5; 54]). In this case, the functional (3.110) is the integral of the moment map in [49, Section 5] and geodesic stability coincides with analytic stability (by [49, Corollary 5.3]).

\subsection{The $\alpha$-Futaki character, the $\alpha-K-$ energy and the geodesic equation}

We now prove that condition $(\star)$ of Section 3.2 is satisfied and give explicit formulae for the character $\mathcal{F}_{I}$, the functional $\mathcal{M}_{I}$ and the geodesic equation on $B_{I}$ introduced in Section 3.3.

Fix a complex structure on $X$ for which $\Omega \in H^{2}(X, \mathbb{R})$ is a Kähler class (ie, it contains a Kähler form) and a holomorphic structure on the principal $G^{c}$-bundle $\pi: E^{c} \rightarrow X$. These data determine a point $I \in \mathcal{Z}$. As explained in Section 3.3, condition (3.102) for the orbit $\Gamma \cdot I$ is equivalent to the existence of a pair $b=(\omega, H) \in B_{I}$ such that the 
point $(J, A) \in \mathcal{P}_{b}$ corresponding to $I$ via Lemma 3.1 satisfies the coupled equations (2.47). In other words, condition (3.102) for the orbit $\Gamma \cdot I$ is equivalent to the existence of a solution $b=(\omega, H) \in B_{I}$ to the following coupled equations, where $S_{\omega}$ is the scalar curvature of the Riemannian metric $g_{\check{I}}=\omega(\cdot, \check{I} \cdot)$ and $F_{H}$ is the curvature of the Chern connection of $H$ and $I$ :

$$
\left.\begin{array}{l}
\Lambda_{\omega} F_{H}=z, \\
\alpha_{0} S_{\omega}+\alpha_{1} \Lambda_{\omega}^{2}\left(F_{H} \wedge F_{H}\right)=c .
\end{array}\right\}
$$

By (3.105), these equations are satisfied if and only if the 1-form $\sigma_{I}$ on $B_{I}$ has a zero. Now, the definition of $\sigma_{I}$ in (3.103), and in fact the whole of Section 3.2, 3.3, depend on condition $(\star)$ of Section 3.2. To prove this condition, note first that by (3.64), there is a canonical isomorphism

$$
T_{b} B_{I} \cong d d^{c} C^{\infty}(X) \oplus \mathbf{i} \Omega^{0}\left(\operatorname{ad} E_{H}\right),
$$

for all $b=(\omega, H) \in B_{I}$, obtained from the $d d^{c}$-lemma and from the pointwise isomorphism i Lie $G \cong G^{c} / G$ induced by the exponential. Define now Lie $\mathcal{G}^{c}=$ $\Omega^{0}\left(\operatorname{ad} E^{c}\right)$ and Lie $\mathcal{G}_{H}=\Omega^{0}\left(\operatorname{ad} E_{H}\right)$ as the Lie algebras of the gauge group $\mathcal{G}^{c}$ of $E^{c}$ and the gauge group $\mathcal{G}_{H}$ of $E_{H}$, respectively. Consider the projection maps onto the real and imaginary parts associated to $H \in \mathcal{R}$,

$$
\operatorname{Re}_{H}, \operatorname{Im}_{H}: \text { Lie } \mathcal{G}^{c} \longrightarrow \text { Lie } \mathcal{G}_{H},
$$

defined by $y=\operatorname{Re}_{H} y+\mathbf{i} \operatorname{Im}_{H} y$ for all $y \in \operatorname{Lie} \mathcal{G}^{c}$, where we are using the canonical isomorphism

$$
\text { Lie } \mathcal{G}^{c} \cong \operatorname{Lie} \mathcal{G}_{H} \oplus \mathbf{i} \text { Lie } \mathcal{G}_{H}
$$

induced by (3.67) and $\mathfrak{g}^{c}=\mathfrak{g} \oplus \mathbf{i} \mathfrak{g}$.

Lemma 3.15 The infinitesimal action of $y \in \operatorname{Lie}\left(\right.$ Aut $E^{c}$ ) on $H \in \mathcal{R}$ is

$$
Y_{y \mid H}=\mathbf{i} \operatorname{Im}_{H}\left(\theta_{A} y\right),
$$

where $\theta_{A}: T E^{c} \rightarrow V E^{c}$ is the vertical projection induced by any connection $A$ on $E_{H}$.

Proof Using the maps $\theta_{A}, \theta_{A}^{\perp}$ in (3.66), any $y \in \operatorname{Lie}\left(\right.$ Aut $E^{c}$ ) can be decomposed as

$$
y=\mathbf{i} \operatorname{Im}_{H}\left(\theta_{A} y\right)+\operatorname{Re}_{H}\left(\theta_{A} y\right)+\theta_{A}^{\perp} \check{y},
$$

where $\check{y}$ is the vector field on $X$ covered by $y$. Hence the flow $g_{-t}$ of $-y$ can be written as

$$
g_{-t}=f_{-t} \circ s_{t}
$$


where $f_{t} \in$ Aut $E_{H}$ is the flow of $\operatorname{Re}_{H}\left(\theta_{A} y\right)+\theta^{\perp} \breve{y}$ and $s_{t}$ is the flow of the timedependent vector field $-f_{t *}\left(\mathbf{i} \operatorname{Im}_{H}\left(\theta_{A} y\right)\right)$. Therefore, using the isomorphism $T_{H} \mathcal{R} \cong$ i $\Omega^{0}\left(\operatorname{ad} E_{H}\right)$ (also used in (3.120)) and the fact that $f_{t}^{-1}$ preserves $H$, we see that the flow $g_{t}$ of $y$ satisfies

$$
Y_{y \mid H}=\frac{d}{d t}{ }_{\mid t=0} g_{t} \cdot H=\frac{d}{d t}{ }_{\mid t=0} s_{t}^{-1} \cdot H=f_{t *}\left(\mathbf{i} \operatorname{Im}_{H}\left(\theta_{A} y\right)\right)_{\mid t=0}=\mathbf{i} \operatorname{Im}_{H}\left(\theta_{A} y\right) .
$$

Proposition 3.16 Condition $(\star)$ is satisfied. The inverse of (3.85) is given by

$$
\zeta_{I}(v)=-\mathbf{i} \dot{H}-\theta \frac{\perp}{H} \eta_{\phi} \in \operatorname{Lie} \widetilde{\mathcal{G}}_{b},
$$

where $v \in T_{b} B_{I}$ corresponds to $\left(d d^{c} \phi, \dot{H}\right) \in d d^{c} C^{\infty}(X) \oplus \mathbf{i} \Omega^{0}\left(\operatorname{ad} E_{H}\right)$ via (3.120).

Proof Fix $b=(\omega, H) \in B_{I}$. Given $\zeta \in$ Lie $\Gamma$ covering a vector field $\breve{\zeta}$ on $X$, we have

$$
\left.Y_{I \zeta \mid b}=(-d(\check{I} \check{\zeta}\lrcorner \omega), \mathbf{i} \operatorname{Re}_{H}\left(\theta_{H} \zeta\right)\right)
$$

by Lemma 3.15, as $I \zeta$ covers $\check{I} \breve{\zeta}$ and $\theta_{H} \circ I=\mathbf{i} \theta_{H}$, where $\theta_{H}: T E^{c} \rightarrow V E^{c}$ is the vertical projection in (3.66) induced by the Chern connection of $I$ on $E_{H}$. In particular, when $\zeta \in$ Lie $\widetilde{\mathcal{G}_{b}}, \breve{\zeta}=\eta_{\phi}$ is the Hamiltonian vector field of some $\phi \in C^{\infty}(X)$ and (3.123) becomes

$$
Y_{I \zeta \mid b}=\left(-d d^{c} \phi, \mathbf{i} \theta_{H} \zeta\right)
$$

Hence, by (3.120) the infinitesimal action (3.124) is in $T_{b} B_{I}$ and so the morphism (3.85) is well defined. Furthermore, (3.124) easily implies that (3.85) has an inverse given by (3.122).

Using the formula (3.122), the 1 -form $\sigma_{I}$ on $B_{I}$ is given by

$$
\sigma_{I}(v)=4 \alpha_{1} \int_{X} \mathbf{i} \dot{H} \wedge\left(\Lambda_{\omega} F_{H}-z\right) \omega^{[n]}+\int_{X} \phi S_{\alpha}(b, I) \omega^{[n]},
$$

for all $v=\left(d d^{c} \phi, \dot{H}\right) \in T_{b} B_{I}$, where $\phi \omega^{[n]}$ has zero integral on $X$ and $S_{\alpha}(b, I)$ is given by (3.78).

The complex character $\mathcal{F}_{I}$ : Lie $\Gamma_{I} \rightarrow \mathbb{C}$ defined by (3.108) provides our first obstruction to the existence of solutions to (3.119). To give an explicit expression for $\mathcal{F}_{I}$, note first that

$$
\text { Lie } \Gamma_{I}=\operatorname{Lie} \operatorname{Aut}\left(E^{c}, I\right)
$$


is the Lie algebra of the automorphism group of the holomorphic bundle $\left(E^{c}, I\right)$, so each $\zeta \in$ Lie $\Gamma_{I}$ covers a real holomorphic vector field $\breve{\zeta}$ on $(X, \breve{I})$. Now, we can write

$$
\check{\zeta}=\eta_{\phi_{1}}+\check{I} \eta_{\phi_{2}}+\beta,
$$

for any given Kähler form $\omega \in \mathcal{K}_{\check{I}}$, where $\eta_{\phi_{j}}$ is the Hamiltonian vector field of $\phi_{j} \in C_{0}^{\infty}(X)$ on $(X, \omega)$, for $j=1,2$, and $\beta$ is the dual of a 1 -form which is harmonic with respect to the Kähler metric $\omega(\cdot, \check{I} \cdot$ ) (see eg [39]). Using this decomposition in (3.123), we see that the infinitesimal action of $\zeta \in \operatorname{Lie} \operatorname{Aut}\left(E^{c}, I\right)$ on $b=(\omega, H) \in B_{I}$ is

$$
Y_{\zeta \mid b}=\left(-d d^{c} \phi_{2}, \mathbf{i} \operatorname{Im}_{H} \theta_{H} \zeta\right)
$$

hence defining the complex-valued function $\phi:=\phi_{1}+\mathbf{i} \phi_{2}$,

$$
\left\langle\mathcal{F}_{I}, \zeta\right\rangle=-4 \alpha_{1} \int_{X} \theta_{H} \zeta \wedge\left(\Lambda_{\omega} F_{H}-z\right) \omega^{[n]}-\int_{X} \phi S_{\alpha}(b, I) \omega^{[n]},
$$

which must vanish if (3.119) has a solution, by Theorem 3.9.

It is now clear from formula (3.126) that for trivial $G^{c}, \mathcal{F}_{I}$ is the Futaki invariant [22] of the Kähler class $\Omega$ on $(X, \check{I})$, up to a multiplicative factor. For non-trivial $G^{c}$ and $\alpha_{0}=0$, the character $\mathcal{F}_{I}$, restricted to the Lie subalgebra of Lie $\Gamma_{I}$ consisting of vector fields covering holomorphic complex Hamiltonian vector fields (ie, vector fields that vanish somewhere on $X$ ), has already been constructed by Futaki (see [23, Theorem 1.1]).

Using now (3.111), the $\alpha-\mathrm{K}$-energy can be written explicitly along a curve $b_{t}=$ $\left(\omega_{t}, H_{t}\right)$ on $B_{I}$, with $\omega_{t}=\omega_{0}+d d^{c} \phi_{t}$ and $\dot{\phi}_{t} \omega_{t}^{[n]}$ with zero integral on $X$, as

$$
\begin{aligned}
\mathcal{M}_{I}\left(b_{t}, b\right)=\mathcal{M}_{I}\left(b_{0}, b\right)+4 \alpha_{1} \int_{0}^{t} \int_{X} \mathbf{i} \dot{H}_{s} & \wedge\left(\Lambda_{\omega_{s}} F_{H_{s}}-z\right) \omega_{s}^{[n]} \wedge d s \\
& +\int_{0}^{t} \int_{X} \dot{\phi}_{S} S_{\alpha}\left(b_{s}, I\right) \omega^{[n]} \wedge d s .
\end{aligned}
$$

By Proposition 3.10, $\mathcal{M}_{I}(\cdot, b)$ is convex along geodesics on the symmetric space $\left(B_{I}, \nabla\right)$. The explicit expression of the affine connection $\nabla$ and its geodesic equation in the coordinates provided by the canonical isomorphism (3.120) are the content of the following proposition.

For the next result, given $b=(\omega, H) \in B_{I}$, we denote by $(\cdot, \cdot)_{\omega}$ the metric on $T^{*} X$ associated to $\omega(\cdot, \breve{I} \cdot)$ and by $d_{H}$ the covariant derivative associated to the Chern connection of $H$ and $I$. 


\section{Proposition 3.17}

(1) The Christoffel symbol $\Gamma: T_{b} B_{I} \times T_{b} B_{I} \rightarrow T_{b} B_{I}$ is

$\left.\left.\Gamma\left(\dot{b}_{1}, \dot{b}_{2}\right)=\left(-d d^{c}\left(d \phi_{1}, d \phi_{2}\right)_{\omega},-\check{I} \eta_{\phi_{1}}\right\lrcorner d_{H} \dot{H}_{2}-\check{I} \eta_{\phi_{2}}\right\lrcorner d_{H} \dot{H}_{1}+\mathbf{i} F_{H}\left(\eta_{\phi_{1}}, \check{I} \eta_{\phi_{2}}\right)\right)$, for all $\dot{b}_{j}=\left(d d^{c} \phi_{j}, \dot{H}_{j}\right) \in T_{b} B_{I}$, with $j=1,2$.

(2) A curve $b_{t}=\left(\omega_{t}, H_{t}\right)$ on $B_{I}$, with $\omega_{t}=\omega+d d^{c} \phi_{t}$, is a geodesic if and only if

$$
\left.\begin{array}{l}
d d^{c}\left(\ddot{\phi}_{t}-\left(d \dot{\phi}_{t}, d \dot{\phi}_{t}\right)_{\omega_{t}}\right)=0, \\
\left.\ddot{H}_{t}-2 \check{I} \eta_{\dot{\phi}_{t}}\right\lrcorner d_{H_{t}} \dot{H}_{t}+\mathbf{i} F_{H_{t}}\left(\eta_{\dot{\phi}_{t}}, \check{I} \eta_{\dot{\phi}_{t}}\right)=0,
\end{array}\right\}
$$

where $\eta_{\dot{\phi}_{t}}$ is the Hamiltonian vector field of $\dot{\phi}_{t}$ over $\left(X, \omega_{t}\right)$.

Proof The proof of part (1) is a computation of the covariant derivative of a vector field $v_{t}=\left(d d^{c} \psi_{t}, \mathbf{i} \xi_{t}\right)$ along a curve $b_{t}=\left(\omega_{t}, H_{t}\right)$ on $B_{I}$, ie, a curve $v_{t}$ on $T B_{I}$ with $v_{t} \in T_{b_{t}} B_{I}$ for all $t$. Recall that the covariant derivative of $v_{t}$ along $b_{t}$ is (see eg [35, page 114])

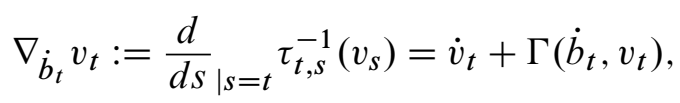

where $\dot{v}_{t}=\left(d d^{c} \dot{\psi}_{t}, \mathbf{i} \dot{\xi}_{t}\right)$ and $\tau_{t, s}: T_{b_{t}} B_{I} \rightarrow T_{b_{s}} B_{I}$ is the parallel transport along $b_{t}$. To calculate (3.129) we compute the parallel transport $\tau_{0, t}(v)$ of any $v=\left(d d^{c} \phi, \dot{H}\right) \in$ $T_{b} B_{I}$ along $b_{t}$ using (3.97). Let $\zeta_{t}=\operatorname{Ad}\left(g_{t}\right) \zeta_{I}(v)$, where $g_{t}$ is the horizontal lift of $b_{t}$ to $\mathcal{Y}_{b_{0}, I}$ (ie, the flow of $\left.I \zeta_{I}\left(\dot{b}_{t}\right)\right)$ with $g_{0}=1$ (see (3.87) and Proposition 3.5). By (3.122),

$\zeta_{t}=\left(g_{t}\right)_{*}\left(-\mathbf{i} \dot{H}-\theta_{\dot{H}, I}^{\perp} \eta_{\phi}\right)=-\mathbf{i}\left(g_{t}\right)_{*} \dot{H}-\theta_{H_{t}, I_{t}}^{\perp}\left(\left(\check{g}_{t}\right)_{*} \eta_{\phi}\right)=-\mathbf{i}\left(g_{t}\right)_{*} \dot{H}-\theta_{H_{t}, I_{t}}^{\perp} \eta_{t}$,

where $\eta_{t}$ is the Hamiltonian vector field of $\phi \circ \check{g}_{t}^{-1}$ over $\left(X, \omega_{t}\right)$, so by (3.97) and (3.123),

$$
\begin{aligned}
\tau_{0, t}(\dot{b}) & =Y_{I \zeta_{t} \mid b_{t}}=\left(d d^{c}\left(\phi \circ \check{g}_{t}^{-1}\right), \mathbf{i} \operatorname{Re}_{H_{t}} \theta_{H_{t}, I}\left(-\mathbf{i}\left(g_{t}\right)_{*} \dot{H}-\theta_{H_{t}, I_{t}} \eta_{t}\right)\right) \\
& \left.=\left(d d^{c}\left(\phi \circ \check{g}_{t}^{-1}\right), \operatorname{Im}_{H_{t}}\left(\left(g_{t}\right)_{*} \dot{H}\right)+\eta_{t}\right\lrcorner \mathbf{i}\left(\theta_{H_{t}, I_{t}}-\theta_{H_{t}, I}\right)\right) .
\end{aligned}
$$

Hence we obtain

$$
\left.\tau_{t, s}^{-1}\left(v_{s}\right)=\left(d d^{c}\left(\psi_{s} \circ \check{g}_{s}\right), \operatorname{Im}_{H_{t}}\left(\mathbf{i}\left(g_{t, s}\right)^{*} \xi_{s}\right)+\eta_{t, s}\right\lrcorner \mathbf{i}\left(\theta_{H_{t}, I_{s}}-\theta_{H_{t}, I}\right)\right),
$$

where $v_{s}=\left(d d^{c} \psi_{s}, \mathbf{i} \xi_{s}\right) \in T_{b_{s}} B_{I}, g_{t, s}$ is the flow of $I \zeta_{I}\left(\dot{b}_{s}\right)$ with $g_{t, t}=1, \eta_{t, s}$ is the Hamiltonian vector field of $\psi_{s} \circ \check{g}_{t, s}$ over $\left(X, \omega_{t}\right)$ and $I_{s}=g_{t, s}^{-1} \cdot I$. Thus denoting 
$\nabla_{\dot{b}_{t}} v_{t}=\left(w_{t}^{1}, w_{t}^{2}\right)$, we conclude that

$$
\begin{aligned}
w_{t}^{1}= & d d^{c}\left(\dot{\psi}_{t}-d \psi_{t}\left(\check{I} \eta_{\dot{\phi}_{t}, \omega_{t}}\right)\right)=d d^{c} \dot{\psi}_{t}-d d^{c}\left(d \psi_{t}, d \dot{\phi}_{t}\right)_{\omega_{t}}, \\
w_{t}^{2}= & \operatorname{Im}_{H_{t}}\left(\mathbf{i} \dot{\xi}_{t}+\left[I \zeta_{I}\left(\dot{b}_{t}\right), \mathbf{i} \xi_{t}\right]\right)-\mathbf{i} \theta_{H_{t}, I}\left[I \zeta_{I}\left(\dot{b}_{t}\right), \theta_{H_{t}, I}^{\perp} \eta_{\psi_{t}}\right] \\
= & \mathbf{i} \dot{\xi}_{t}+\operatorname{Im}_{H_{t}}\left[I \zeta_{I}\left(\dot{b}_{t}\right), \mathbf{i} \xi_{t}\right]+\mathbf{i} \theta_{H_{t}, I}\left[\zeta_{I}\left(\dot{b}_{t}\right), \theta_{H_{t}, I}^{\perp}\left(\check{I} \eta_{\psi_{t}}\right)\right] \\
= & \dot{v}_{t}^{2}+\operatorname{Im}_{H_{t}}\left[\dot{H}_{t}, \mathbf{i} \xi_{t}\right]-\left[\theta_{H_{t}, I}^{\perp}\left(\check{I} \eta_{\dot{\phi}_{t}}\right), \mathbf{i} \xi_{t}\right]-\left[\theta_{H_{t}, I}^{\perp}\left(\check{I} \eta_{\psi_{t}}\right), \dot{H}_{t}\right] \\
& \quad-\mathbf{i} \theta_{H_{t}, I}\left[\theta_{\dot{H}_{t}, I}^{\perp} \eta_{\dot{\phi}_{t}}, \theta_{H_{t}, I}^{\perp}\left(\check{I} \eta_{\psi_{t}}\right)\right] \\
= & \left.\left.\mathbf{i} \dot{\xi}_{t}-\check{I} \eta_{\dot{\phi}_{t}}\right\lrcorner d_{H_{t}}\left(\mathbf{i} \xi_{t}\right)-\check{I} \eta_{\psi_{t}}\right\lrcorner d_{H_{t}} \dot{H}_{t}+\mathbf{i} F_{H_{t}}\left(\eta_{\dot{\phi}_{t}}, \check{I} \eta_{\psi_{t}}\right) .
\end{aligned}
$$

This proves (1). Note that $F_{H}$ and $\omega$ are of type $(1,1)$, so the torsion is $T_{\nabla}=0$ (cf Theorem 3.6) and the geodesic equation is (3.128). This proves (2).

Remark 3.18 When $G^{c}$ is the trivial group, so $E^{c}=X$, Theorem 3.6 and Proposition 3.17 reduce to the corresponding results for the space of Kähler metrics $\mathcal{K}_{\check{I}}$ already studied by Mabuchi [41; 42] and Donaldson [18]. More precisely, we recover the Levi-Civita connection of the Mabuchi metric on the Riemannian symmetric space $\mathcal{K}_{\check{I}}$, the functional $\mathcal{M}_{I}(\cdot, b)$ is the Mabuchi K-energy [41; 42] on the space of Kähler metrics, by formula (3.127), and (3.128) reduces to the geodesic equation on the space of Kähler metrics [42]

$$
\ddot{\phi}_{t}-\left(d \dot{\phi}_{t}, d \dot{\phi}_{t}\right)_{\omega_{t}}=0
$$

It seems plausible that the methods used by Chen [10], and Chen and Tian [13] in their study of (3.131) could be adapted to equation (3.128) and to the existence and uniqueness problem for the coupled equations. As in the case of (3.131), this would require a reformulation of (3.128) as a complex Monge-Ampère equation.

Note that the explicit formula for the Christoffel symbols in Proposition 3.17 provides a direct proof of the vanishing of the torsion $T_{\nabla}$ (cf Theorem 3.6). Observe also that the two factors of $B_{I}=\mathcal{K}_{\check{I}} \times \mathcal{R}$ are Riemannian symmetric spaces with holonomy groups contained in $\mathcal{H}_{\omega}$ (see $\left[42 ; 18\right.$, Section 4]) and $\mathcal{G}_{H}$, and that the holonomy group of $B_{I}$ is contained in their group extension $\widetilde{\mathcal{G}}_{b}$ (see (1.26)). Here, the structure of Riemannian symmetric space on $\mathcal{R}$ depends on the choice of an element $\omega \in \mathcal{K}_{\check{I}}$. However, Proposition 3.17 implies that the symmetric space structure of $B_{I}$ is not the product structure. In fact, it is an open question whether $B_{I}$ carries a Riemannian metric compatible with $\nabla$ (see Remark 4.4 for details). 


\section{Extremal pairs and deformation of solutions}

Following the approach of LeBrun and Simanca [38; 39], in this section we define an extremality condition for pairs $(\omega, H)$ and also an extremality condition in the weak coupling limit (see (4.132) and (4.183)). We establish existence results for extremal pairs $(\omega, H)$ near solutions to the coupled equations under deformations of the coupling constants and the Kähler class (Theorems 4.10 and 4.17) and find sufficient conditions for the existence of solutions to the coupled equations (Theorems 4.11 and 4.18).

In Section 4 we fix a complex reductive Lie group $G^{c}$, an $n$-dimensional compact complex manifold $(X, J)$, with underlying real manifold $X$ and complex structure $J$, and a holomorphic principal $G^{c}$-bundle $\left(E^{c}, I\right)$ over $(X, J)$ with underlying real principal $G^{c}$-bundle $E^{c}$ and complex structure $I$ (so $\breve{I}=J$ in the notation of Section 3.1). We also fix a maximal compact Lie subgroup $G \subset G^{c}$. The Lie algebras of $G \subset G^{c}$ are denoted $\mathfrak{g} \subset \mathfrak{g}^{c}$, respectively. As in Theorem 3.2, we fix a $G^{c}$-invariant symmetric bilinear form $(\cdot, \cdot): \mathfrak{g}^{c} \otimes \mathfrak{g}^{c} \rightarrow \mathbb{C}$, which restricts to a $G$-invariant positive definite inner product on $\mathfrak{g}$. Finally, $\mathfrak{z}=\mathfrak{g}^{G}$ and $\mathfrak{z}^{c}=\left(\mathfrak{g}^{\mathfrak{c}}\right)^{G^{c}}$ denote the subsets of elements of $\mathfrak{g}$ and $\mathfrak{g}^{c}$ that are invariant under the adjoint actions of $G$ and $G^{c}$, respectively $(\mathrm{cf}(1.15))$.

\subsection{Extremal pairs}

We start studying an extremality condition which will be useful to prove Theorem 4.11. Throughout Section 4.1, we fix $\alpha=\left(\alpha_{0}, \alpha_{1}\right) \in \mathbb{R}^{2}$ such that $\alpha_{1} \neq 0$, and a Kähler class $\Omega$ on $(X, J)$. Note that we will not assume $\alpha_{0}, \alpha_{1}>0$, but that we can still apply Proposition 3.8 and Theorem 3.9 (see Remark 3.12). We define

$$
B_{\Omega}:=\mathcal{K}_{\Omega} \times \mathcal{R}
$$

where $\mathcal{K}_{\Omega}$ is the space of Kähler forms in $\Omega$ compatible with the complex structure $J$ and $\mathcal{R}=\Omega^{0}\left(E^{c} / G\right)(\operatorname{cf}(3.64))$.

The following definition is closely related to the vanishing condition for the linearisation at a solution of the coupled equations (see Proposition 4.7 and Lemma 4.8).

Definition 4.1 A pair $b=(\omega, H) \in B_{\Omega}$ is extremal if it satisfies the equations

$$
\left.\begin{array}{l}
\left.4 \alpha_{1} d_{H} \Lambda_{\omega} F_{H}+\eta_{\alpha}(b)\right\lrcorner F_{H}=0, \\
L_{\eta_{\alpha}(b)} J=0,
\end{array}\right\}
$$

where $\eta_{\alpha}(b)$ is the Hamiltonian vector field on $(X, \omega)$ of the function

$$
S_{\alpha}(b):=-\alpha_{0} S_{\omega}-\alpha_{1} \Lambda_{\omega}^{2}\left(F_{H} \wedge F_{H}\right)+4 \alpha_{1} \Lambda_{\omega} F_{H} \wedge z_{\Omega} \in C^{\infty}(X),
$$


(cf (3.78)). Here $S_{\omega}$ is the scalar curvature of the metric $\omega(\cdot, J \cdot)$ and $z_{\Omega}$ is the element of $\mathfrak{z} \subset \mathfrak{g}$ given by (1.18).

Extremal Kähler metrics in $\Omega$, introduced by Calabi in [8, Section 1], can be characterised as those $\omega \in \mathcal{K}_{\Omega}$ such that the Hamiltonian vector field of $S_{\omega}$ over $(X, \omega)$ is in Lie $\mathcal{H}_{J}$ [8, Section 2]. In particular, all cscK metrics are extremal. Similarly, extremal pairs admit a description in terms of real-holomorphic vector fields on the total space of $E^{c}$. To see this, recall that each $H \in \mathcal{R}$ induces a reduction of $E^{c}$ to a principal $G$-bundle $E_{H} \subset E^{c}$ (see Section 3.1) and each $b=(\omega, H) \in B_{\Omega}$ determines a short exact sequence of Lie groups (see Section 1.2)

$$
1 \longrightarrow \mathcal{G} \longrightarrow \tilde{\mathcal{G}} \stackrel{p}{\longrightarrow} \mathcal{H} \longrightarrow 1,
$$

given from left to right by the gauge group of $E_{H}$, the extended gauge group of $E_{H}$ over $(X, \omega)$ and the group of Hamiltonian symplectomorphisms of $(X, \omega)$. This exact sequence induces another one

$$
1 \longrightarrow \mathcal{G}_{I} \longrightarrow \widetilde{\mathcal{G}}_{I} \stackrel{p}{\longrightarrow} \mathcal{H}_{J},
$$

where $\mathcal{G}_{I}=\mathcal{G} \cap \operatorname{Aut}\left(E^{c}, I\right), \widetilde{\mathcal{G}}_{I}=\widetilde{\mathcal{G}} \cap \operatorname{Aut}\left(E^{c}, I\right)$ and $\mathcal{H}_{J}=\mathcal{H} \cap \operatorname{Aut}(X, J)$ are finite-dimensional complex Lie groups (see eg [6, Section 2.120]). Note that the Lie algebra Lie $\widetilde{\mathcal{G}}_{I}$ is given by $G^{c}$-invariant real-holomorphic vector fields on the total space of $\left(E^{c}, I\right)$ covering Hamiltonian (real-holomorphic) vector fields on $(X, J, \omega)$. Using the horizontal lift $\theta_{H}^{\perp}:$ Lie $\mathcal{H} \rightarrow$ Lie $\widetilde{\mathcal{G}}_{b}$ of the Chern connection associated to $H$ and $I$ (cf (3.66)), we define

$$
\zeta_{\alpha}(b):=-4 \alpha_{1}\left(\Lambda_{\omega} F_{H}-z_{\Omega}\right)-\theta_{H}^{\perp} \eta_{\alpha}(b) \in \text { Lie } \widetilde{\mathcal{G}}_{b},
$$

for each $b=(H, \omega) \in B_{\Omega}$. Then it follows from (1.30) and (3.70) that

$$
b \in B_{\Omega} \text { is extremal } \Longleftrightarrow \zeta_{\alpha}(b) \in \operatorname{Lie} \tilde{\mathcal{G}}_{I} .
$$

The following link between extremal pairs and the coupled equations is a generalization of the corresponding link for Kähler metrics (see eg [39, Lemma 1]). To establish this, note that each $\omega \in \mathcal{K}_{\Omega}$ induces $L^{2}$-inner products on $C^{\infty}(X)$ and $\Omega^{0}\left(\operatorname{ad} E_{H}\right)$, given by

$$
\begin{aligned}
\left\langle\phi_{0}, \phi_{1}\right\rangle_{\omega} & :=\int_{X} \phi_{0} \phi_{1} \omega^{[n]}, \\
\left\langle\xi_{0}, \xi_{1}\right\rangle_{\omega} & :=\int_{X}\left(\xi_{0} \wedge \xi_{1}\right) \omega^{[n]},
\end{aligned}
$$

for $\phi_{j} \in C^{\infty}(X), \xi_{j} \in \Omega^{0}\left(\operatorname{ad} E_{H}\right)(j=0,1)$. Their associated $L^{2}$-norms are denoted $\|\cdot\|_{\omega}$. 
Proposition 4.2 A pair $b \in B_{\Omega}$ is a solution to the coupled equations (3.119) if and only if it is an extremal pair and $\mathcal{F}_{\alpha, \Omega}=0$.

Proof If there exists a solution $b=(\omega, H) \in B_{\Omega}$ to the coupled equations (3.119), then $\mathcal{F}_{\alpha, \Omega}=0$, by (3.105) (or (3.126)) and Theorem 3.9, and furthermore, $b$ is obviously an extremal pair, since $d_{H} \Lambda_{\omega} F_{H}=0$ and $\eta_{\alpha}(b)=0$. Conversely, if $b=(\omega, H)$ is extremal, ie, $\zeta_{\alpha}(b) \in$ Lie $\widetilde{\mathcal{G}} I$, then

$$
\mathcal{F}_{\alpha, \Omega}\left(\zeta_{\alpha}(b)\right)=\left\|S_{\alpha}(b)-\widehat{S}_{\alpha}\right\|_{\omega}^{2}+16 \alpha_{1}^{2}\left\|\Lambda_{\omega} F_{H}-z_{\Omega}\right\|_{\omega}^{2} \geq 0,
$$

by (3.126), where $\widehat{S}_{\alpha}=\int_{X} S_{\alpha}(b) \omega^{[n]} / \operatorname{Vol}_{\Omega}$, so $\mathcal{F}_{\alpha, \Omega}=0$ implies that $b$ satisfies (3.119).

Extremal pairs enjoy good regularity properties, similar to those of extremal Kähler metrics [38, Proposition 4]:

Lemma 4.3 Let $(\omega, H)$ be an extremal pair such that $\omega$ is a Kähler form of class $C^{2}$ on $(X, J)$ and $H$ is a section of $E^{c} / G$ of class $C^{4}$. Then both $\omega$ and $H$ are smooth.

Proof We will show by induction on $l \in \mathbb{N}$ that $\omega$ and $H$ are Hölder of class $C^{2 l-1, \beta}$ and $C^{2 l+1, \beta}$ respectively, for all $\beta \in(0,1)$ and $l \in \mathbb{N}$. By assumption, $\omega$ and $H$ are of class $C^{1, \beta}$ and $C^{3, \beta}$, respectively. Suppose now that $\omega$ and $H$ are of class $C^{2 l-1, \beta}$ and $C^{2 l+1, \beta}$, respectively. As $\eta_{\alpha}(b)$ is a real holomorphic vector field by (4.137), it is real analytic, so $d S_{\alpha}(b)$ is of class $C^{2 l-1, \beta}$, ie, $S_{\alpha}(b) \in C^{2 l, \beta}$, and hence it follows from (4.133) that the scalar curvature $S_{\omega}$ is of class $C^{2 l-1, \beta}$, because

$$
\Lambda_{\omega}^{2}\left(F_{H} \wedge F_{H}\right)-4 \Lambda_{\omega} F_{H} \wedge z_{\Omega}
$$

is of class $C^{2 l-1, \beta}$. Arguing as in [38, Proposition 4], it follows from the regularity theory for the Laplacian and for the Monge-Ampère equation that $\omega$ is of class $C^{2 l+1, \beta}$ (recall that the scalar curvature can be written in holomorphic coordinates as $\left.\Delta_{\omega} \log \operatorname{det}(\omega)\right)$. Since $\zeta_{\alpha}(b)$, defined by (4.136), is a real-holomorphic vector field on $\left(E^{c}, I\right)$ by assumption (see (4.137)), it is real analytic and so $\Lambda_{\omega} F_{H}$ is of class $C^{2 l, \beta}$, because $\alpha_{1} \neq 0$. Identifying $H$ locally with a function on the base with values in $\exp (\mathbf{i} \mathfrak{g}) \subset G^{c}$ and using holomorphic coordinates for the bundle $E^{c}$, we can write

$$
\Delta_{\bar{\partial}} H=H\left(\Lambda_{\omega} F_{H}-\Lambda_{\omega}\left(\bar{\partial}\left(H^{-1}\right) \wedge \partial H\right)\right),
$$

where the right-hand side is of class $C^{2 l, \beta}$ and $\Delta_{\bar{\partial}}$ is elliptic with $C^{2 l+1, \beta}$ coefficients. By the regularity theory of linear elliptic differential operators, $H$ is of class $C^{2 l+2, \beta}$ (see eg Aubin [5, Theorem 3.55]). Applying this argument again to (4.136), we see that the right-hand side of (4.139) is of class $C^{2 l+1, \beta}$ and so $H$ is of class $C^{2 l+3, \beta}$, as required. 
Remark 4.4 Note that $B_{\Omega}=\mathcal{K}_{\Omega} \times \mathcal{R}$ has a Riemannian metric $g_{\Omega}$, given by

$$
g_{\Omega}\left(v_{0}, v_{1}\right)=\int_{X} \phi_{0} \phi_{1} \omega^{[n]}+\int_{X}\left(\xi_{0} \wedge \xi_{1}\right) \omega^{[n]}
$$

for $b=(\omega, H), v_{j}=\left(\phi_{j}, \xi_{j}\right) \in T_{b} B_{\Omega} \cong C_{0}^{\infty}(X) \times \Omega^{0}\left(\operatorname{ad} E_{H}\right)(\mathrm{cf}(4.141))$, with $\dot{H}=\mathbf{i} \xi$ in the notation of Section 3.4. Although this metric is rather canonical, it does not endow the symmetric space $\left(B_{\Omega}, \nabla\right)$ of Theorem 3.6 with a structure of Riemannian symmetric space, since $g_{\Omega}$ is not preserved in general by the canonical affine connection $\nabla$ on $B_{\Omega}$ constructed in Section 3.2. In fact, by a straightforward calculation using formula (3.130) for the parallel transport,

$$
\begin{aligned}
& \left(\nabla_{v_{0}} g_{\Omega}\right)\left(v_{1}, v_{2}\right) \\
& \quad=-\int_{X}\left(\dot{\xi}_{1} \wedge\left(\theta_{H}\left[\zeta_{I}\left(v_{0}\right), \theta_{H}^{\perp} J \eta_{\phi_{2}}\right]\right)+\left(\theta_{H}\left[\zeta_{I}\left(v_{0}\right), \theta_{H}^{\perp} J \eta_{\phi_{1}}\right]\right) \wedge \dot{\xi}_{2}\right) \omega^{[n]} .
\end{aligned}
$$

However, if the group $G^{c}$ is trivial, so $B_{\Omega}=\mathcal{K}_{\Omega}$, then $g_{\Omega}$ is precisely the Mabuchi metric and $\nabla g_{\Omega}=0$, by the previous formula, so we recover the known fact [18; 42] that $\mathcal{K}_{\Omega}$ is a Riemannian symmetric space with Levi-Civita connection $\nabla$, by Theorem 3.6.

\subsection{Holomorphic vector fields on the principal bundle}

Given $b=(\omega, H) \in B_{\Omega}$, we now relate the Lie algebra Lie $\widetilde{\mathcal{G}}_{I}$ (see (4.135)) to the space of solutions to a fourth-order elliptic differential equation that is closely related to the linearization of our coupled equations. We will use the inner product on $C^{\infty}(X) \times \Omega^{0}\left(\operatorname{ad} E_{H}\right)$ induced by (4.138), ie, given by

$$
\left\langle v_{0}, v_{1}\right\rangle_{\omega}:=\left\langle\phi_{0}, \phi_{1}\right\rangle_{\omega}+\left\langle\xi_{0}, \xi_{1}\right\rangle_{\omega},
$$

for $v_{j}=\left(\phi_{j}, \xi_{j}\right) \in C^{\infty}(X) \times \Omega^{0}\left(\operatorname{ad} E_{H}\right)(j=0,1)$.

We define an operator:

$$
\begin{aligned}
\mathrm{P}=\mathrm{P}_{\omega}: C^{\infty}(X) & \longrightarrow \Omega^{0}(\text { End } T X), \\
\phi & \longmapsto-L_{\eta_{\phi}} J .
\end{aligned}
$$

In other words, $\mathrm{P}$ is induced by the infinitesimal action of Lie $\mathcal{H}$ on $\mathcal{J}_{\omega}$. Let $\mathrm{P}^{*}$ be the formal adjoint of $\mathrm{P}$ with respect to the $L^{2}$-inner products on $C^{\infty}(X)$ and $\Omega^{0}$ (End $\left.T X\right)$ induced by $\omega(\cdot, J \cdot)$, with the $L^{2}$-inner product on $\Omega^{0}$ (End $\left.T X\right)$ multiplied by a factor of $\frac{1}{2}$, so that its restriction to $T_{J} \mathcal{J}$ coincides with $\omega_{\mathcal{J}}(\cdot, \mathbf{J} \cdot)$ (defined by (2.40)). Then $\mathrm{P}^{*} \mathrm{P}$ is, up to a multiplicative constant factor, the Lichnerowicz operator of the compact Kähler manifold $(X, J, \omega)$. This is an elliptic self-adjoint semipositive differential operator of order 4 , whose kernel is the set of functions $\phi$ such that $\eta_{\phi} \in$ Lie $\mathcal{H}_{J}$, and which may be interpreted as the linearization of the cscK equation at $\omega$ (see eg [38]). 
We define now an operator that is closely related to the linearization of the coupled equations (see Proposition 4.7) and that will play the role of the Lichnerowicz operator in our study. The operator is

$$
\mathbf{L}_{\alpha, b}=\left(\mathbf{L}_{\alpha, b}^{0}, \mathbf{L}_{\alpha, b}^{1}\right): C^{\infty}(X) \times \Omega^{0}\left(\operatorname{ad} E_{H}\right) \longrightarrow C^{\infty}(X) \times \Omega^{0}\left(\operatorname{ad} E_{H}\right),
$$

where $\mathbf{L}_{\alpha, b}^{0}$ and $\mathbf{L}_{\alpha, b}^{1}$ are defined by

$$
\begin{array}{r}
\left.\mathbf{L}_{\alpha, b}^{0}(\phi, \xi)=\alpha_{0} \mathrm{P}^{*} \mathrm{P} \phi-2 \alpha_{1} \Lambda_{\omega}^{2}\left(F_{H} \wedge d_{H} J\left(d_{H} \xi+\eta_{\phi}\right\lrcorner F_{H}\right)\right) \\
+\mathbf{L}_{\alpha, b}^{1}(\phi, \xi) \wedge z_{\Omega}, \\
\left.\left.\mathbf{L}_{\alpha, b}^{1}(\phi, \xi)=4 \alpha_{1} \Lambda_{\omega} d_{H} J\left(d_{H} \xi+\eta_{\phi}\right\lrcorner F_{H}\right)=4 \alpha_{1} d_{H}^{*}\left(d_{H} \xi+\eta_{\phi}\right\lrcorner F_{H}\right) .
\end{array}
$$

Here, $J$ is the endomorphism of $\Omega^{1}\left(\operatorname{ad} E_{H}\right)$ induced by the complex structure $J$ (see [6, (2.8)] $), d_{H}: \Omega^{0}\left(\operatorname{ad} E_{H}\right) \rightarrow \Omega^{1}\left(\operatorname{ad} E_{H}\right)$ is the covariant derivative of the Chern connection of $H$ and $I$ and, by the Kähler identities, $d_{H}^{*}=\Lambda_{\omega} d_{H} J$ is its formal adjoint.

Recall that the Chern connection associated to any $H \in \mathcal{R}$ and $I$ induces a vector space isomorphism (see Section 1.3):

$$
C^{\infty}(X) / \mathbb{R} \times \Omega^{0}\left(\operatorname{ad} E_{H}\right) \stackrel{\cong}{\longrightarrow} \operatorname{Lie} \widetilde{\mathcal{G}}_{b}:([\phi], \xi) \longmapsto \xi+\theta_{H}^{\perp} \eta_{\phi}
$$

Let $\left(Z_{b}, \mathbf{I}_{b}, \omega_{b}\right)$ be the Kähler manifold constructed in Lemma 3.1 and Theorem 3.2.

Lemma 4.5 Let $v_{j}=\left(\phi_{j}, \xi_{j}\right) \in C^{\infty}(X) \times \Omega^{0}\left(\operatorname{ad} E_{H}\right)$, for $j=0,1$. Then

$$
\begin{aligned}
\left\langle v_{0}, \mathbf{L}_{\alpha, b} v_{1}\right\rangle_{\omega}=\omega_{b}\left(Y_{\zeta_{0} \mid I},\right. & \left.\mathbf{I} Y_{\zeta_{1} \mid I}\right) \\
& \left.\left.+4 \alpha_{1}\left\langle J \eta_{\phi_{0}}\right\lrcorner\left(d_{H} \xi_{1}+\eta_{\phi_{1}}\right\lrcorner F_{H}\right), \Lambda_{\omega} F_{H}-z_{\Omega}\right\rangle_{\omega},
\end{aligned}
$$

where $Y_{\zeta_{j}}$ is the infinitesimal action of $\zeta_{j}=\xi_{j}+\theta_{H}^{\perp} \eta_{\phi_{j}} \in \operatorname{Lie} \widetilde{\mathcal{G}}_{b}$ on $Z_{b}$, for $j=0,1$.

Proof By the moment map interpretation of scalar curvature of the Kähler metric $\omega(\cdot, J \cdot)$, its derivative $\delta_{J} S_{\omega}: T_{J} \mathcal{J}_{\omega} \rightarrow C^{\infty}(X)$ with respect to $J \in \mathcal{J}_{\omega}$ satisfies

$$
\delta_{J} S_{\omega} \circ \mathbf{J} \circ \mathrm{P}=-\mathrm{P}^{*} \mathrm{P}
$$

(see Section 2.1 and Donaldson [19, Equation (26)]). By (3.81) and Lemma 3.1, I $Y_{\zeta_{0} \mid I}$ is identified with the infinitesimal action of $I \zeta_{0}$ on $(J, A) \in \mathcal{P}_{b}$, where $A$ is the Chern connection of $H$ and $I$, so

$$
\left.\mathbf{I} Y_{\zeta_{1} \mid I}=-L_{I \zeta_{1}} I=\left(-J L_{\eta_{\phi_{1}}} J, J\left(d_{H} \xi_{1}+\eta_{\phi_{1}}\right\lrcorner F_{H}\right)\right),
$$

by Lemma 1.5. Hence (4.146) follows from formula (2.44) for the moment map $\mu_{b}$. 
Given a pair $b=(\omega, H) \in B_{\Omega}$, an element $\zeta \in \operatorname{Lie} \widetilde{\mathcal{G}}_{b}$ is in Lie $\widetilde{\mathcal{G}}_{I}$ if and only if $Y_{\zeta \mid I}=0$. Using (4.148), we see that Lie $\widetilde{\mathcal{G}}_{I} \subset$ Lie $\widetilde{\mathcal{G}}_{b}$ is the subset of elements $\zeta=\xi+\theta \frac{\perp}{H} \eta_{\phi}$ such that

$$
\left.\mathrm{P} \phi=0, \quad d_{H} \xi+\eta_{\phi}\right\lrcorner F_{H}=0 .
$$

Hence if $(\phi, \xi) \in C^{\infty}(X) \times \Omega^{0}\left(\operatorname{ad} E_{H}\right)$ satisfies $\xi+\theta_{H}^{\perp} \eta_{\phi} \in$ Lie $\widetilde{\mathcal{G}}_{I}$, then it is $\operatorname{ker} \mathbf{L}_{\alpha, b}$ (see (4.144)). We provide now sufficient conditions to obtain the converse implication.

Given a pair $b=(\omega, H) \in B_{\Omega}, H$ is a Hermitian-Yang-Mills reduction (HYM) on $\left(E^{c}, I\right)$ with respect to $\omega$ if it satisfies

$$
\Lambda_{\omega} F_{H}=z_{\Omega} .
$$

Note that if $H$ is HYM on $\left(E^{c}, I\right)$ with respect to $\omega$, then (4.146) becomes simply

$$
\left\langle v_{0}, \mathbf{L}_{\alpha, b} v_{1}\right\rangle_{\omega}=\omega_{b}\left(Y_{\zeta_{0} \mid I}, \mathbf{I} Y_{\zeta_{1} \mid I}\right)
$$

Proposition 4.6 The operator $\mathbf{L}_{\alpha, b}$ is elliptic. If $H$ is $H Y M$ with respect to $\omega$, then $\mathbf{L}_{\alpha, b}$ is also self-adjoint. If furthermore $\alpha_{0} \alpha_{1}>0$, then

$$
\operatorname{ker} \mathbf{L}_{\alpha, b}=\left\{(\phi, \xi) \in C^{\infty}(X) \times \Omega^{0}\left(\operatorname{ad} E_{H}\right) \mid \xi+\theta_{H}^{\perp} \eta_{\phi} \in \operatorname{Lie} \widetilde{\mathcal{G}}_{I}\right\} .
$$

Proof The operator $\mathbf{L}_{\alpha, b}$ is elliptic because so are $\mathrm{P}^{*} \mathrm{P}$ and $d_{H}^{*} d_{H}$. If $H$ is HYM, then we can apply $(4.151)$, where $\omega_{b}(\cdot, \mathbf{I} \cdot)$ is symmetric, so $\mathbf{L}_{\alpha, b}$ is self-adjoint. We have already seen that the right-hand side of (4.152) is contained in $\operatorname{ker} \mathbf{L}_{\alpha, b}$. If in addition $\alpha_{0} \alpha_{1}>0$, then $\omega_{b}$ is compatible with either $\mathbf{I}$ or $-\mathbf{I}$ (see Section 2.2), so if $v=(\phi, \xi)$ satisfies $\zeta:=\xi+\theta_{H}^{\perp} \eta_{\phi} \notin \operatorname{Lie} \widetilde{\mathcal{G}}_{I}$, then $\left\langle v, \mathbf{L}_{\alpha, b} v\right\rangle_{\omega}=\omega_{b}\left(Y_{\zeta \mid I}, \mathbf{I} Y_{\zeta \mid I}\right) \neq 0$ by (4.151), and hence $v \notin \operatorname{ker} \mathbf{L}_{\alpha, b}$. This implies (4.152).

Observe that, although $\mathbf{L}_{\alpha, b}$ is an analogue in our context of the Lichnerowicz operator, there is an important difference between these two operators, since by Proposition 4.6, we can ensure that $\mathbf{L}_{\alpha, b}$ is self-adjoint and its kernel corresponds to Lie $\widetilde{\mathcal{G}}_{I}$ via (4.145) only when $b=(\omega, H)$ satisfies the Hermitian-Yang-Mills equation (4.150) and $\alpha_{0} \alpha_{1}>0$.

\subsection{The linearised coupled equations}

Throughout Section 4.3, we fix a coupling constant $\alpha \in \mathbb{R}^{2}$, a holomorphic structure $I$ on $E^{c}$ over $(X, J)$, a Kähler class $\Omega$ on $(X, J)$ and $b=(\omega, H) \in B_{\Omega}$. Let $H^{1,1}(X, \mathbb{R}) \subset H^{2}(X, \mathbb{R})$ be the vector subspace of those de Rham classes which are representable by real closed $(1,1)$-forms on $(X, J)$. Recall that $H^{1,1}(X, \mathbb{R})$ is 
identified by Hodge theory with the space $\mathcal{H}^{1,1}(X)$ of real harmonic $(1,1)$-forms on $(X, J, \omega)$.

In Section 4.3, we will compute the first-order deformations of the moment map $\mu_{b}$ constructed in Theorem 3.2 under deformations given by a new Kähler form $\widetilde{\omega}$ and a new holomorphic structure $\tilde{I}$ on the principal bundle $E^{c}$ over $(X, J)$, given by

$$
\begin{aligned}
\widetilde{\omega} & :=\omega+\gamma+d d^{c} \phi, \\
\widetilde{I} & :=e^{\mathbf{i} \xi} \cdot I,
\end{aligned}
$$

parametrised by a triple

$$
(\gamma, \phi, \xi) \in \mathcal{H}^{1,1}(X) \times C^{\infty}(X) \times \Omega^{0}\left(\operatorname{ad} E_{H}\right) .
$$

We will also consider the deformed pair

$$
\widetilde{b}=(\widetilde{\omega}, \widetilde{H}) \in B_{\widetilde{\omega}} \quad \text { with } \widetilde{H}:=e^{-\mathbf{i} \xi} \cdot H \in \mathcal{R},
$$

where $\widetilde{\omega}$ is the cohomology class of $\widetilde{\omega}$. Note that (3.77) implies

$$
F_{H, \tilde{I}}=e^{\mathbf{i} \xi} \cdot F_{\tilde{H}, I},
$$

where $F_{H, I}$ is the curvature of the Chern connection $\theta_{H}=\theta_{H, I}$ associated to $H$ and $I$.

In fact, to prove Theorems 4.11 and 4.18 , we will need to apply the implicit function theorem, so we will work in Sobolev spaces. Let $L_{k}^{2}(X)$ and $L_{k}^{2}\left(\operatorname{ad} E_{H}\right)$ be the Sobolev spaces of real-valued functions on $X$ and sections of the bundle ad $E_{H}$, respectively, whose distributional derivatives up to order $k$ are square integrable. These are real Hilbert spaces that, by the Sobolev embedding theorem, have natural bounded inclusion maps $L_{k}^{2}(X) \subset C^{l}(X)$ and $L_{k}^{2}\left(\operatorname{ad} E_{H}\right) \subset C^{l}\left(\operatorname{ad} E_{H}\right)$ into the Banach spaces of $l$-times continuously differentiable functions and sections of ad $E_{H}$, respectively, provided $k>n+l$. Moreover, if $k>n$, then $L_{k}^{2}(X)$ is a Banach algebra. Fix $k>n$. Let

$$
\mathcal{U}=\widehat{\mathcal{U}} \times L_{k+4}^{2}\left(\operatorname{ad} E_{H}\right) \subset \mathcal{H}^{1,1}(X) \times L_{k+4}^{2}(X) \times L_{k+4}^{2}\left(\operatorname{ad} E_{H}\right),
$$

where $\widehat{\mathcal{U}} \subset \mathcal{H}^{1,1}(X) \times L_{k+4}^{2}(X)$ is the open neighbourhood of $(0,0)$ consisting of pairs $(\gamma, \phi)$ such that $\widetilde{\omega}(\cdot, J \cdot)$ is a Kähler metric of class $C^{2}$, with $\widetilde{\omega}$ defined by (4.153a). Define the moment map operator

$$
\begin{aligned}
\mathrm{T}_{\alpha}=\left(\mathrm{T}_{\alpha}^{0}, \mathrm{~T}_{\alpha}^{1}\right): \mathcal{U} & \longrightarrow L_{k}^{2}(X) \times L_{k+2}^{2}\left(\operatorname{ad} E_{H}\right), \\
(\gamma, \phi, \xi) & \longmapsto\left(S_{\alpha}(\tilde{b}, I), 4 \alpha_{1}\left(\Lambda_{\widetilde{\omega}} F_{H, \tilde{I}}-z_{\widetilde{\omega}}\right)\right),
\end{aligned}
$$


where $\widetilde{\omega}, \widetilde{I}$ and $\tilde{b}$ are defined by (4.153) and (4.154), while $S_{\alpha}(\tilde{b}, I)$ and $z_{\widetilde{\omega}}$ are given by the formulae (4.133) and (4.150), using the Kähler class

$$
\widetilde{\omega}:=[\widetilde{\omega}] \in H^{1,1}(X, \mathbb{R}) .
$$

Observe that $T_{\alpha}$ is a variant for Sobolev spaces of the families of moment maps $\mu_{b}$. The following proposition can be compared with [39, Proposition 5].

Proposition 4.7 For $k>n, \mathrm{~T}_{\alpha}$ is a well-defined $C^{1}$ map whose Fréchet derivative $\delta \mathrm{T}_{\alpha}$ at the origin $(0,0,0)$ is given by

$$
\left.=\mathbf{L}_{\alpha, b}(\dot{\phi}, \dot{\xi})+\left(\left(d\left(S_{\alpha}(b, I)\right), d \dot{\phi}\right)_{\omega}, 4 \alpha_{1} J \eta_{\dot{\phi}}\right\lrcorner d_{H} \Lambda_{\omega} F_{H}\right)+\delta_{\dot{\gamma}} \mathrm{T}_{\alpha},
$$

for all $(\dot{\gamma}, \dot{\phi}, \dot{\xi}) \in \mathcal{H}^{1,1}(X) \times L_{k+4}^{2}(X) \times L_{k}^{2}\left(\operatorname{ad} E_{H}\right)$, where

$$
\mathbf{L}_{\alpha, b}: L_{k+4}^{2}(X) \times L_{k+4}^{2}\left(\operatorname{ad} E_{H}\right) \longrightarrow L_{k}^{2}(X) \times L_{k+2}^{2}\left(\operatorname{ad} E_{H}\right)
$$

is given by (4.144), $(\cdot, \cdot)_{\omega}$ is the inner product on $T^{*} X$ induced by $\omega(\cdot, J \cdot), \eta_{\dot{\phi}}$ is the Hamiltonian vector field of $\dot{\phi}$ on $(X, \omega)$ and $\delta_{\dot{\gamma}} \mathrm{T}_{\alpha}$ is the directional derivative of $\mathrm{T}_{\alpha}$ at the origin in the direction $(\dot{\gamma}, 0,0)$.

Proof The operator $\mathrm{T}_{\alpha}$ is well-defined because $L_{k}^{2}(X)$ is a Banach algebra for $k>n$, $\mathrm{T}_{\alpha}^{0}$ is a non-linear differential operator of order 4 in $\phi$ and order 2 in $\gamma$ and $\xi$, while $\mathrm{T}_{\alpha}^{1}$ is a non-linear differential operator of order 2 in $\phi$ and $\xi$ and order 0 in $\gamma$.

To prove that $\mathrm{T}_{\alpha}$ is $C^{1}$, we will calculate its directional derivatives

$$
\delta_{(\dot{\phi}, \dot{\xi})} \mathrm{T}_{\alpha}(\gamma, \phi, \xi)
$$

and $\delta_{\dot{\gamma}} \mathrm{T}_{\alpha}(\gamma, \phi, \xi)$ at $(\gamma, \phi, \xi)$ in the directions $(0, \dot{\phi}, \dot{\xi})$ and $(\dot{\gamma}, 0,0)$, respectively, for $(\gamma, \phi, \xi) \in \mathcal{U},(\dot{\gamma}, \dot{\phi}, \dot{\xi}) \in \mathcal{H}^{1,1}(X) \times L_{k+4}^{2}(X) \times L_{k}^{2}\left(\operatorname{ad} E_{H}\right)$.

To compute $\delta_{(\dot{\phi}, \dot{\xi})} \mathrm{T}_{\alpha}(\gamma, \phi, \xi)$, we define a curve (on an appropriate Sobolev completion of $B_{\Omega}$ and for $|t|$ small), given by

$$
b_{t}=\left(\widetilde{\omega}_{t}, \widetilde{H}_{t}\right):=\left(\widetilde{\omega}+t d d^{c} \dot{\phi}, e^{-\mathbf{i}(\xi+t \dot{\xi})} \cdot H\right) .
$$

Let $\eta_{t}$ be the Hamiltonian vector field of $\dot{\phi}$ over $\left(X, \widetilde{\omega}_{t}\right)$ and $g_{t}$ the flow of

$$
y_{t}:=I \zeta_{I}\left(\dot{b}_{t}\right)=-I\left(\dot{\xi}+\theta_{\dot{H}_{t}}^{\perp} \eta_{t}\right)
$$


ie, the curve of $G^{c}$-equivariant automorphisms of $E^{c}$ satisfying $\dot{g}_{t} \cdot g_{t}^{-1}=y_{t}$, with initial condition $g_{0}=\mathrm{Id}$. Since the Kähler class $\widetilde{\omega}$ of $\widetilde{\omega}_{t}$ is constant along the curve $b_{t}$, we can apply the constructions in the proof of Proposition 3.5(1), so the flow $g_{t}$ exists and satisfies

$$
b_{t}=g_{t} \cdot \tilde{b}
$$

(as $b_{0}=\tilde{b}$ ). Note that the identity (4.160) holds in a strong sense, as $k>n$, so the Kähler metrics $\widetilde{\omega}_{t}$ are of class $C^{2}$ and the $G$-reductions $\widetilde{H}_{t}$ are of class $C^{4}$. Define another curve

$$
I_{t}:=g_{t}^{-1} \cdot I
$$

in (an appropriate Sobolev completion of) the space $Z_{\widetilde{b}}$ of holomorphic structures on the principal $G^{c}$-bundle $E^{c}$ that are compatible with $\tilde{b}$ (see Section 3.1). Using the dependence of $S_{\alpha}\left(b_{t}, I\right)$ on the holomorphic structure $I$ on $E^{c}$, we obtain

$$
\mathrm{T}_{t}^{0}:=\mathrm{T}_{\alpha}^{0}(\gamma, \phi+t \dot{\phi}, \xi+t \dot{\xi})=S_{\alpha}\left(b_{t}, I\right)=S_{\alpha}\left(\tilde{b}, I_{t}\right) \circ \check{g}_{t}^{-1}
$$

by (3.79) and (4.160). Since $\left.\frac{d}{d t}\right|_{t=0} I_{t}=L_{y_{0}} I$, this implies

$$
\begin{aligned}
\delta_{(\dot{\phi}, \dot{\xi})} \mathrm{T}_{\alpha}^{0}(\gamma, \phi, \xi) & \left.=\left.\frac{d}{d t}\right|_{\mid t=0} \mathrm{~T}_{t}^{0}=\left(\delta_{I} S_{\alpha}\right)_{\mid(\widetilde{b}, I)}\left(L_{y_{0}} I\right)+J \eta_{\dot{\phi}}\right\lrcorner d\left(S_{\alpha}(\tilde{b}, I)\right) \\
& =\left(\delta_{I} S_{\alpha}\right)_{\mid(\widetilde{b}, I)}\left(L_{y_{0}} I\right)+\left(d\left(S_{\alpha}(\tilde{b}, I)\right), d \dot{\phi}\right)_{\widetilde{\omega}}
\end{aligned}
$$

where $\delta_{I} S_{\alpha}: T_{I} Z_{\widetilde{b}} \rightarrow C^{\infty}(X)$ is the derivative of $S_{\alpha}$ with respect to $I$. Now, by (4.148)

$$
\left.L_{y_{0}} I=\left(-J L_{\eta_{\dot{\phi}}} J, J\left(d_{\tilde{H}} \dot{\xi}+\eta_{\dot{\phi}}\right\lrcorner F_{\tilde{H}}\right)\right),
$$

and from this formula, (4.147) and (4.144), we obtain

$$
\begin{aligned}
\left(\delta_{I} S_{\alpha}\right)_{\mid(\widetilde{b}, I)}\left(\mathbf{I}_{\xi_{\dot{b}}}\right) & \\
& =\alpha_{0} \mathrm{P}^{*} \mathrm{P} \dot{\phi}-2 \alpha_{1} \Lambda_{\widetilde{\omega}}^{2}\left(F_{\widetilde{H}} \wedge d_{\widetilde{H}} J\left(d_{\widetilde{H}} \dot{\xi}+\eta_{\dot{\phi}}\right\lrcorner F_{\widetilde{H}}\right)+\mathbf{L}_{\alpha, \widetilde{b}}^{1}(\dot{\phi}, \dot{\xi}) \wedge z_{\widetilde{\omega}} \\
& =\mathbf{L}_{\alpha, \widetilde{b}}^{0}(\dot{\phi}, \dot{\xi}),
\end{aligned}
$$

where $z_{\widetilde{\omega}}$ is defined as in (4.133) using the Kähler class $\widetilde{\omega}$, so the right hand side of (4.161) is

$$
\delta_{(\gamma, \phi, \xi)} \mathrm{T}_{\alpha}^{0}(0, \dot{\phi}, \dot{\xi})=\mathbf{L}_{\alpha, \widetilde{b}}^{0}(\dot{\phi}, \dot{\xi})+\left(d\left(S_{\alpha}(\widetilde{b}, I)\right), d \dot{\phi}\right) \widetilde{\omega}
$$

By (3.77), we also have

$$
\mathrm{T}_{t}^{1}:=\mathrm{T}_{\alpha}^{1}\left(\gamma, \phi+\dot{\phi}_{t}, \xi+t \dot{\xi}\right)=4 \alpha_{1}\left(e^{\mathrm{i}(\xi+t \dot{\xi})} g_{t}\right) \cdot\left(\Lambda_{\widetilde{\omega}_{t}} F_{H, I_{t}}-z \widetilde{\omega}\right),
$$


and a straightforward calculation shows that

$$
\begin{aligned}
\delta_{(\dot{\phi}, \dot{\xi})} & \mathrm{T}_{\alpha}^{1}(\gamma, \phi, \xi) \\
= & \left.\left.\left.\frac{d}{d t}\right|_{t=0} \mathrm{~T}_{t}^{1}=4 \alpha_{1} \Lambda_{\widetilde{\omega}} d_{\widetilde{H}} J\left(d_{\widetilde{H}} \dot{\xi}+\eta_{\dot{\phi}}\right\lrcorner F_{\tilde{H}}\right)+4 \alpha_{1} J \eta_{\dot{\phi}}\right\lrcorner d_{\widetilde{H}} \Lambda_{\widetilde{\omega}} F_{\tilde{H}} \\
= & \left.\mathbf{L}_{\alpha, b}^{1}(\dot{\phi}, \dot{\xi})+4 \alpha_{1} J \eta_{\dot{\phi}}\right\lrcorner d_{\widetilde{H}} \Lambda_{\widetilde{\omega}} F_{\tilde{H}} .
\end{aligned}
$$

To compute $\delta_{\dot{\gamma}} \mathrm{T}_{\alpha}(\gamma, \phi, \xi)$, for $(\gamma, \phi, \xi) \in \mathcal{U}$ and $\dot{\gamma} \in \mathcal{H}^{1,1}(X)$, we define a curve

$$
b_{t}=\left(\omega_{t}, \widetilde{H}\right)=(\widetilde{\omega}+t \dot{\gamma}, \widetilde{H})
$$

(for $t \in \mathbb{R}$ small). Let

$$
\begin{aligned}
\mathrm{T}_{t}^{0} & :=\mathrm{T}_{\alpha}^{0}(\gamma+t \dot{\gamma}, \phi, \xi)=S_{\alpha}\left(b_{t}, I\right) \\
& =-\alpha_{0} S_{\omega_{t}}-\alpha_{1} \Lambda_{\omega_{t}}^{2}\left(F_{\tilde{H}} \wedge F_{\tilde{H}}\right)+4 \alpha_{1} \Lambda_{\omega_{t}} F_{\tilde{H}} \wedge z_{\Omega_{t}}, \\
\mathrm{~T}_{t}^{1} & :=\mathrm{T}_{\alpha}^{1}(\gamma+t \dot{\gamma}, \phi, \xi)=4 \alpha_{1}\left(\Lambda_{\omega_{t}} F_{H, \tilde{I}}-z_{\Omega_{t}}\right),
\end{aligned}
$$

where $\Omega_{t}=\left[\omega_{t}\right] \in H^{1,1}(X, \mathbb{R})$. As shown by LeBrun and Simanca (see [39, Proposition 5] and [38, Proposition 6]), the derivative of the first term of $\mathrm{T}_{t}^{0}$ is given by

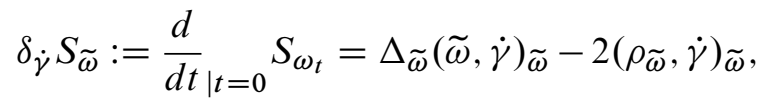

where $\Delta_{\widetilde{\omega}}$ and $\rho_{\widetilde{\omega}}$ are the Laplacian and the Ricci curvature of $\widetilde{\omega}(\cdot, J \cdot)$, respectively. To calculate the derivatives of $\mathrm{T}_{t}^{1}$ and of the second term of $\mathrm{T}_{t}^{0}$, we use the equality

$$
\frac{d}{d t}_{\mid t=0} \omega_{t}^{[n]}=\dot{\gamma} \wedge \widetilde{\omega}^{[n-1]}=\left(\Lambda_{\widetilde{\omega}} \dot{\gamma}\right) \widetilde{\omega}^{[n]}
$$

and the following computations:

$$
\begin{aligned}
& \frac{d}{d t}{ }_{\mid t=0}\left(\Lambda_{\omega_{t}} F_{H, \widetilde{I}^{\omega_{t}}}^{[n]}\right)=\frac{d}{d t}{ }_{\mid t=0}\left(F_{H, \tilde{I}} \wedge \omega_{t}^{[n-1]}\right)=F_{H, \widetilde{I}} \wedge \dot{\gamma} \wedge \widetilde{\omega}^{[n-2]}, \\
& \left.\frac{d}{d t}\right|_{t=0}\left(\left(\Lambda_{\omega_{t}}^{2}\left(F_{\widetilde{H}} \wedge F_{\widetilde{H}}\right)-4 \Lambda_{\omega_{t}} F_{\widetilde{H}} \wedge z_{\Omega_{t}}\right) \omega_{t}^{[n]}\right) \\
& =\left.\frac{d}{d t}\right|_{t=0}\left(2 F_{\tilde{H}} \wedge F_{\tilde{H}} \wedge \omega_{t}^{[n-2]}-4 F_{\widetilde{H}} \wedge z_{\Omega_{t}} \omega_{t}^{[n-1]}\right) \\
& =2 F_{\tilde{H}} \wedge F_{\tilde{H}} \wedge \dot{\gamma} \wedge \widetilde{\omega}^{[n-3]}-4 F_{\tilde{H}} \wedge\left(z_{\tilde{\omega}} \dot{\gamma}+\frac{\delta_{\dot{\gamma}} z \widetilde{\omega} \widetilde{\omega}}{n-1}\right) \wedge \widetilde{\omega}^{[n-2]} .
\end{aligned}
$$

Here, (4.150) implies

$$
\delta_{\dot{\gamma}} z_{\widetilde{\omega}}:=\frac{d}{d t}{ }_{\mid t=0} z_{\Omega_{t}}=\sum_{j} \beta_{j} z_{j}
$$


for an orthonormal basis $\left\{z_{j}\right\}$ of $\mathfrak{z}$, with:

$$
\begin{aligned}
\beta_{j} & :=\frac{d}{d t}{ }_{\mid t=0} \frac{\left\langle z_{j}(E) \cup \Omega_{t}^{[n-1]},[X]\right\rangle}{\operatorname{Vol}_{\Omega_{t}}} \\
& =\frac{\left\langle z_{j}(E) \cup[\dot{\gamma}] \cup \widetilde{\omega}^{[n-2]},[X]\right\rangle}{\operatorname{Vol}_{\tilde{\omega}}}-\frac{\left\langle z_{j}(E) \cup \widetilde{\omega}^{[n-1]},[X]\right\rangle\left\langle[\dot{\gamma}] \cup \widetilde{\omega}^{[n-1]},[X]\right\rangle}{\operatorname{Vol}_{\widetilde{\omega}}^{2}} .
\end{aligned}
$$

From these equalities, we obtain the directional derivatives:

(4.165a) $\delta_{\dot{\gamma}} \mathrm{T}_{\alpha}^{0}(\gamma, \phi, \xi)=\left.\frac{d}{d t}\right|_{t=0} \mathrm{~T}_{t}^{0}=\alpha_{0}\left(2\left(\rho_{\tilde{\omega}}, \dot{\gamma}\right)_{\widetilde{\omega}}-\Delta_{\widetilde{\omega}}(\widetilde{\omega}, \dot{\gamma})_{\widetilde{\omega}}\right)$

$$
\begin{aligned}
& -\frac{\alpha_{1}}{3} \Lambda_{\widetilde{\omega}}^{3}\left(F_{H, \widetilde{I}} \wedge F_{H, \widetilde{I}} \wedge \dot{\gamma}\right)+2 \alpha_{1} \Lambda_{\widetilde{\omega}}^{2}\left(F_{H, \widetilde{I}} \wedge\left(z_{\widetilde{\omega}} \dot{\gamma}+\frac{\delta_{\dot{\gamma}} z \widetilde{\omega} \widetilde{\omega}}{n-1}\right)\right) \\
& +\alpha_{1}\left(\Lambda_{\widetilde{\omega}}^{2}\left(F_{H, \widetilde{I}} \wedge F_{H, \widetilde{I}}\right)-4 \Lambda_{\widetilde{\omega}} F_{\widetilde{H}} \wedge z_{\widetilde{\omega}}\right)\left(\Lambda_{\widetilde{\omega}} \dot{\gamma}\right),
\end{aligned}
$$

(4.165b) $\delta_{\dot{\gamma}} \mathrm{T}_{\alpha}^{1}(\gamma, \phi, \xi)=\frac{d}{d t}{ }_{t=0} \mathrm{~T}_{t}^{1}=4 \alpha_{1}\left(\left(F_{H, \tilde{I}}, \dot{\gamma}\right) \widetilde{\omega}-\delta_{\dot{\gamma}} z_{\widetilde{\omega}}\right)$.

It now follows from (4.161), (4.163), (4.165) and the formula (4.164) for $\delta_{\dot{\gamma}} z_{\widetilde{\omega}}$ that the directional derivatives are continuous. Therefore, $\mathrm{T}_{\alpha}$ is $C^{1}$ and its Fréchet derivative given by (4.158) (by (4.161) and (4.163)).

Note that an explicit formula for the directional derivative $\delta_{\dot{\gamma}} \mathrm{T}_{\alpha}$ has been calculated in (4.165), although it has not been recorded in (4.158), as it is not needed in this paper.

\subsection{Deformation of solutions}

As in Section 4.3, we now fix a holomorphic structure $I$ on $E^{c}$ over $(X, J)$, a Kähler class $\Omega$ on $(X, J)$ and $b=(\omega, H) \in B_{\Omega}$. Note that $\mathcal{H}_{J}$ acts trivially on the space $\mathcal{H}^{1,1}(X) \subset \Omega^{2}(X)$ of real harmonic $(1,1)$-forms for the metric $\omega(\cdot, J \cdot)$. Let

$$
L_{k}^{2}(X)^{\mathcal{H}_{J}} \subset L_{k}^{2}(X) \text { and } L_{k}^{2}\left(\operatorname{ad} E_{H}\right)^{\widetilde{\mathcal{G}}_{I}} \subset L_{k}^{2}\left(\operatorname{ad} E_{H}\right)
$$

be the closed subspaces of $\mathcal{H}_{J}$-invariant functions and $\widetilde{\mathcal{G}}_{I}$-invariant sections, respectively. Let $\hat{\mathcal{V}}=\widehat{\mathcal{U}} \cap\left(\mathcal{H}^{1,1}(X) \times L_{k}^{2}(X)^{\mathcal{H}_{J}}\right)$ and

$$
\text { (4.167) } \mathcal{V}=\hat{\mathcal{V}} \times L_{k}^{2}\left(\operatorname{ad} E_{H}\right)^{\widetilde{\mathcal{G}}_{I}}=\mathcal{U} \cap\left(\mathcal{H}^{1,1}(X) \times L_{k+4}^{2}(X)^{\mathcal{H}_{J}} \times L_{k+4}^{2}\left(\operatorname{ad} E_{H}\right)^{\widetilde{\mathcal{G}}_{I}}\right) \text {. }
$$

Given coupling constants $\alpha \in \mathbb{R}^{2}$, by restriction of the maps of Proposition 4.7, for $k>n$, we obtain well-defined maps

(4.168a) $\quad \widehat{\mathrm{T}}_{\alpha}: \mathcal{V} \longrightarrow L_{k}^{2}(X)^{\mathcal{H}_{J}} \times L_{k+2}^{2}\left(\operatorname{ad} E_{H}\right)^{\widetilde{\mathcal{G}}_{I}}$,

(4.168b) $\hat{\mathbf{L}}_{\alpha, b}: L_{k+4}^{2}(X)^{\mathcal{H}_{J}} \times L_{k+4}^{2}\left(\operatorname{ad} E_{H}\right)^{\widetilde{\mathcal{G}}_{I}} \longrightarrow L_{k}^{2}(X)^{\mathcal{H}_{J}} \times L_{k+2}^{2}\left(\operatorname{ad} E_{H}\right)^{\widetilde{\mathcal{G}}_{I}}$, 
(cf [38, (5.1)]), where $\widehat{\mathrm{T}}_{\alpha}$ is $C^{1}$ with Fréchet derivative given by (4.158), and $\hat{\mathbf{L}}_{\alpha, b}$ is a linear elliptic operator.

Let $d^{*}$ and $\mathbf{G}$ be the formal adjoint of the de Rham differential and the Green operator of the Laplacian for the fixed metric $\omega(\cdot, J \cdot)$, respectively. Then for any symplectic form $\widetilde{\omega}$ and any $\tilde{\eta}$ in the Lie algebra Lie $\mathcal{H}_{\tilde{\omega}}$ of Hamiltonian vector fields over $(X, \widetilde{\omega})$ we have

$$
\left.\left.d\left(\mathbf{G} d^{*}(\tilde{\eta}\lrcorner \tilde{\omega}\right)\right)=\tilde{\eta}\right\lrcorner \tilde{\omega} .
$$

As the image of the Green operator is perpendicular to the constants, the Hamiltonian function $\left.f=\mathbf{G} d^{*}(\tilde{\eta}\lrcorner \widetilde{\omega}\right)$ is "normalized" for the volume form $\omega^{[n]}$, that is, $\int_{X} f \omega^{[n]}=0$.

For each $(\gamma, \phi, \xi) \in \mathcal{V}$, we define a linear map

$$
\begin{aligned}
\mathbf{P}_{(\gamma, \phi, \xi)}=\left(\mathbf{P}_{(\gamma, \phi)}^{0}, \mathbf{P}_{\xi}^{1}\right): \mathbb{R} \times \mathfrak{z}\left(\operatorname{Lie} \widetilde{\mathcal{G}}_{I}\right) & \longrightarrow L_{k}^{2}(X)^{\mathcal{H} J} \times L_{k+2}^{2}\left(\operatorname{ad} E_{H}\right)^{\widetilde{\mathcal{G}}_{I}} \\
(t, v) & \left.\longmapsto\left(\mathbf{G} d^{*}(p(v)\lrcorner \widetilde{\omega}\right)+t, \theta_{H, \widetilde{I}} v\right),
\end{aligned}
$$

where

$$
\mathfrak{z}\left(\operatorname{Lie} \widetilde{\mathcal{G}}_{I}\right):=\left(\operatorname{Lie} \widetilde{\mathcal{G}}_{I}\right)^{\widetilde{\mathcal{G}}_{I}}
$$

is the centre of Lie $\widetilde{\mathcal{G}}_{I}(\operatorname{cf}(1.15))$ and $p: \widetilde{\mathcal{G}}_{I} \rightarrow \mathcal{H}_{J}$ is the map in (4.135), while $\widetilde{\omega}$ and $\widetilde{H}$ are defined by (4.153a) and (4.154). The map $\mathbf{P}_{(\gamma, \phi, \xi)}$ attaches to a vector field $v \in \mathfrak{z}\left(\right.$ Lie $\left.\widetilde{\mathcal{G}}_{I}\right)$ its vertical part $\theta_{H, \tilde{I}} v$, calculates the normalized Hamiltonian function of the vector field $p(v)$ over $(X, \widetilde{\omega})$, and adds an extra parameter $t$, which accounts for the fact that Hamiltonian functions are only determined up to a constant (cf (4.145), [38, Section 5; 39, Proposition 2]).

Here is the key link between extremal pairs and the linearization of the coupled equations.

Lemma 4.8 Let $(\gamma, \phi, \xi) \in \mathcal{V}$.

(1) $\mathbf{P}_{(\gamma, \phi, \xi)}$ is injective.

(2) If $\widehat{\mathrm{T}}_{\alpha}(\gamma, \phi, \xi) \in \operatorname{Im} \mathbf{P}_{(\gamma, \phi, \xi)}$, then $\tilde{b}=(\widetilde{\omega}, \tilde{H})$ is an extremal pair.

(3) $\operatorname{Im} \mathbf{P}_{0} \subset \operatorname{ker} \hat{\mathbf{L}}_{\alpha, b}$, with equality if $\alpha_{0} \alpha_{1}>0$ and $H$ is HYM with respect to $\omega$.

Proof We first prove that, given $(t, v) \in \mathbb{R} \times \mathfrak{z}\left(\operatorname{Lie} \widetilde{\mathcal{G}}_{I}\right)$ and $(f, \chi):=\mathbf{P}_{(\gamma, \phi, \xi)}(t, v)$, we have

$$
t=\int_{X} f \omega^{[n]} / \mathrm{Vol}_{\Omega}, \quad v=\chi+\theta_{H, \tilde{I}}^{\perp} \tilde{\eta}_{f},
$$


where $\tilde{\eta}_{f}$ is the Hamiltonian vector field associated to $f \in C^{\infty}(X)$ and $\tilde{\omega}$. To see this, note that, since $p(v)$ is holomorphic and preserves $\widetilde{\omega}$, it can be written as

$$
p(v)=\tilde{\eta}_{\psi}+\beta,
$$

where $\tilde{\eta}_{\psi}$ is the real-holomorphic Hamiltonian vector field associated to $\psi \in C^{\infty}(X)$ and $\widetilde{\omega}$, and $\beta$ is a parallel vector field with respect to $\widetilde{\omega}$ (see [39, Section 2]). Then, since $p(v)$ and $\tilde{\eta}_{\psi}$ vanish somewhere on $X$, we have that $\beta=0$ and therefore

$$
d \psi=p(v)\lrcorner \tilde{\omega}=d f .
$$

Formula (4.171) follows from the decomposition of $v$ into its vertical and horizontal parts with respect to $\theta_{H, \tilde{I}}$.

Now, (1) follows from (4.171). To prove (2), suppose $\widehat{\mathrm{T}}_{\alpha}(\gamma, \phi, \xi) \in \operatorname{Im} \mathbf{P}_{(\gamma, \phi, \xi)}$, ie,

$$
f=S_{\alpha}(\tilde{b}), \quad \chi=\theta_{h, \widetilde{I}} v=4 \alpha_{1}\left(\Lambda_{\widetilde{\omega}} F_{H, \tilde{I}}-z_{\widetilde{\omega}}\right) .
$$

From (4.171), it follows that

$$
\left.4 \alpha_{1} d_{\widetilde{H}} \Lambda_{\widetilde{\omega}} F_{\widetilde{H}}=-\widetilde{\eta}_{f}\right\lrcorner F_{\widetilde{H}}, \quad \mathrm{P}_{\widetilde{\omega}} f=-L_{\tilde{\eta}_{f}} J=0,
$$

where we have used (3.77) to obtain the first equation, while the other identity follows because $\widetilde{\eta}_{\psi}=\tilde{\eta}_{f}$ is real-holomorphic. Therefore $\widetilde{b}=(\widetilde{\omega}, \widetilde{H})$ is an extremal pair.

To prove (3), note first that the inclusion $\operatorname{Im} \mathbf{P}_{0} \subset \operatorname{ker} \hat{\mathbf{L}}_{\alpha, b}$ is a straightforward consequence of (4.171). Suppose now that $\alpha_{0} \alpha_{1}>0$ and $H$ is HYM with respect to $\omega$. Let $(f, \chi) \in \operatorname{ker} \hat{\mathbf{L}}_{\alpha, b}$. By Proposition 4.6, $v:=\chi+\theta_{H, I}^{\perp} \eta_{f}$ is in Lie $\widetilde{\mathcal{G}}_{I}$. In fact, $v \in \mathfrak{z}\left(\right.$ Lie $\left.\widetilde{\mathcal{G}}_{I}\right)$, as $f$ is $\mathcal{H}_{J}$-invariant and $\chi$ is $\widetilde{\mathcal{G}}_{I}$-invariant by assumption (see (4.168b)). Therefore $\mathbf{P}_{0}(v, t)=(f, \chi)$, where $t:=\int_{X} f \omega^{[n]} / \operatorname{Vol}_{\Omega}$.

Let $\langle\cdot, \cdot\rangle_{\omega}$ be the $L^{2}$-inner product on $L_{k}^{2}(X)^{\mathcal{H}} \times L_{k+2}^{2}\left(\operatorname{ad} E_{H}\right)^{\widetilde{\mathcal{G}}_{I}}$ given by (4.141). We claim that the orthogonal projectors onto $\operatorname{Im} \mathbf{P}_{(\gamma, \phi, \xi)}$, denoted

$$
\Pi_{(\gamma, \phi, \xi)}: L_{k}^{2}(X)^{\mathcal{H}_{J}} \times L_{k+2}^{2}\left(\operatorname{ad} E_{H}\right)^{\widetilde{\mathcal{G}}_{I}} \longrightarrow L_{k}^{2}(X)^{\mathcal{H}_{J}} \times L_{k+2}^{2}\left(\operatorname{ad} E_{H}\right)^{\widetilde{\mathcal{G}}_{I}}
$$

vary smoothly with $(\gamma, \phi, \xi) \in \mathcal{V}$. To prove this, note that the map

$$
\begin{aligned}
\mathbf{P}: \mathcal{V} \times \mathbb{R} \times \mathfrak{z}\left(\operatorname{Lie} \widetilde{\mathcal{G}_{I}}\right) & \longrightarrow L_{k}^{2}(X)^{\mathcal{H}_{J}} \times L_{k+2}^{2}\left(\operatorname{ad} E_{H}\right)^{\tilde{\mathcal{G}}_{I}}, \\
(\gamma, \phi, \xi, t, v) & \longmapsto \mathbf{P}_{(\gamma, \phi, \xi)}(t, v),
\end{aligned}
$$

is $C^{1}$, as $\mathbf{P}_{(\gamma, \phi)}^{0}(t, v)$ is linear in $(\gamma, \phi, t, v)$ and $\mathbf{P}_{\xi}^{1}(v)$ depends linearly on $v$ and smoothly on $\xi$. Moreover, $\mathbf{P}_{(\gamma, \phi, \xi)}$ is an isomorphism onto its image for all $(\gamma, \phi, \xi) \in$ $\mathcal{V}$, by Lemma 4.8. Let $\left\{w_{j}\right\}$ be a basis of the vector space $\mathbb{R} \oplus \mathfrak{z}\left(\right.$ Lie $\left.\widetilde{\mathcal{G}}_{I}\right)$ and $\left\{\zeta_{j}(\gamma, \phi, \xi)\right\}$ be the orthonormal basis of $\operatorname{Im} \mathbf{P}_{(\gamma, \phi, \xi)}$ extracted from $\left\{\mathbf{P}_{(\gamma, \phi, \xi)} w_{j}\right\}$ by 
the Gram-Schmidt orthogonalization process. Then the claim follows by the above observations and the fact that

$$
\Pi_{(\gamma, \phi, \xi)}=\sum_{j}\left\langle\zeta_{j}(\gamma, \phi, \xi), \cdot\right\rangle_{\omega} \zeta_{j}
$$

Furthermore, since $\left\langle\zeta_{j}, \zeta_{k}\right\rangle_{\omega}$ are continuous functions on $\mathcal{V}$, the origin has an open neighbourhood $\mathcal{V}_{0} \subset \mathcal{V}$ such that for all $(\gamma, \phi, \xi) \in \mathcal{V}_{0}$, the following holds (cf [39, (5.3)]):

$$
\operatorname{ker}\left(\mathrm{Id}-\Pi_{(\gamma, \phi, \xi)}\right)=\operatorname{ker}\left(\mathrm{Id}-\Pi_{0}\right) \circ\left(\mathrm{Id}-\Pi_{(\gamma, \phi, \xi)}\right) .
$$

For any pair of non-negative integers $(l, m)$, let $I_{l, m} \subset L_{l}^{2}(X)^{\mathcal{H} J} \times L_{m}^{2}\left(\operatorname{ad} E_{H}\right)^{\tilde{\mathcal{G}}_{I}}$ be the orthogonal complement of $\operatorname{Im} \mathbf{P}_{0}$. Define

$$
\mathcal{W}=\mathcal{V}_{0} \cap\left(\mathcal{H}^{1,1}(X) \times I_{k+4, k+4}\right) .
$$

Note that, under the assumptions in the last part of Lemma 4.8, the subspace $\mathcal{W}$ is perpendicular to $\operatorname{ker} \mathbf{L}_{\alpha, b}$. We will use this fact to obtain existence results about deformations of extremal pairs. Define a LeBrun-Simanca map [38, Section 5]:

$$
\begin{aligned}
\mathbf{T}_{\alpha}: \mathcal{W} & \longrightarrow I_{k, k+2}, \\
(\gamma, \phi, \xi) & \longmapsto\left(\mathrm{Id}-\Pi_{0}\right) \circ\left(\mathrm{Id}-\Pi_{(\gamma, \phi, \xi)}\right) \circ \widehat{\mathrm{T}}_{\alpha}(\gamma, \phi, \xi) .
\end{aligned}
$$

Then $\mathbf{T}_{\alpha}$ is $C^{1}$, because it is the composition of $C^{1}$ maps.

Given $(\dot{\phi}, \dot{\xi}) \in I_{k+4, k+4}$, to calculate the directional derivative $\delta_{(\dot{\phi}, \dot{\xi})} \mathbf{T}_{\alpha}$ of $\mathbf{T}_{\alpha}$ at the origin in the direction $(0, \dot{\phi}, \dot{\xi})$, we define the curve

$$
b_{t}=(0, t \dot{\phi}, t \dot{\xi}) \text {. }
$$

Using (4.158), we obtain:

$$
\begin{aligned}
\delta_{(\dot{\phi}, \dot{\xi})} \mathbf{T}_{\alpha}=\frac{d}{d t} & \mathbf{T}_{\alpha}\left(b_{t}\right)_{\mid t=0} \\
=\left(\operatorname{Id}-\Pi_{0}\right) \mathbf{L}_{\alpha, b}(\dot{\phi}, \dot{\xi}) & \\
& \left.\quad+\left(\operatorname{Id}-\Pi_{0}\right)\left(\left(d\left(S_{\alpha}(b)\right), d \dot{\phi}\right)_{\omega}, 4 \alpha_{1} J \eta_{\dot{\phi}}\right\lrcorner d_{H}\left(\Lambda_{\omega} F_{H}\right)\right) \\
& \quad-\left(\operatorname{Id}-\Pi_{0}\right) \frac{d}{d t}\left(\Pi_{b_{t}} \mathrm{~T}_{\alpha}(0)\right)_{\mid t=0}
\end{aligned}
$$

Now, if $b=(\omega, H)$ is a solution to the coupled equations (3.119), then the second summand of the right-hand side vanishes and $\Pi_{b_{t}} \mathrm{~T}_{\alpha}(0)=\mathrm{T}_{\alpha}(0)$ for all $t$, so the third summand of the right-hand side vanishes too and hence, under this assumption, we 
conclude that

$$
\delta_{(\dot{\phi}, \dot{\xi})} \mathbf{T}_{\alpha}=\left(\mathrm{Id}-\Pi_{0}\right) \circ \widehat{\mathbf{L}}_{\alpha, b}(\dot{\phi}, \dot{\xi})
$$

Remark 4.9 It is at this point that one runs into technical difficulties if one attempts to apply the approach of LeBrun and Simanca [38] to obtain deformations of an extremal pair which is not a solution of the coupled equations. The problem is that for an arbitrary extremal pair $b=(\omega, H)$, if one proceeds as in [38, Lemma 1], then one obtains

$$
\left.\left.\delta_{(\dot{\phi}, \dot{\xi})} \mathbf{T}_{\alpha}=\left(\operatorname{Id}-\Pi_{0}\right)\left(\widehat{\mathbf{L}}_{\alpha, b}(\dot{\phi}, \dot{\xi})+\left(0,-J \eta_{\alpha}(b, I)\right\lrcorner\left(d_{H} \dot{\xi}+\eta_{\dot{\phi}}\right\lrcorner F_{H}\right)\right)\right),
$$

and to construct deformations of $b$ which are also extremal pairs using the approach of [38], we need know that (4.177) is satisfied. A natural condition which implies that (4.177) holds is that $S_{\alpha}(\omega, H)$ is constant. Furthermore, in the approach of [38], we need to know that $\hat{\mathbf{L}}_{\alpha, b}$ is self-adjoint, with kernel $\operatorname{Im} \mathbf{P}_{0}$, so another natural condition is that the Hermitian-Yang-Mills equation is satisfied, by Proposition 4.6 and Lemma 4.8(2). In other words, to get a direct generalization of the method of [38], it is natural to impose the condition that $b$ is a solution of the coupled equations, as we will do below.

We can now prove the two main results of Section 4.4. For this, given $\alpha \in \mathbb{R}^{2}$, we call $b \in B_{\Omega}$ an extremal pair with coupling constants $\alpha$ if it satisfies (4.132).

Theorem 4.10 Suppose $(\omega, H)$ is a solution to the coupled equations (3.119) with coupling constant $\alpha$ and $[\omega]=\Omega$, where $\alpha=\left(\alpha_{0}, \alpha_{1}\right) \in \mathbb{R}^{2}$ satisfies $\alpha_{0} \alpha_{1}>0$. Then $(\alpha, \Omega)$ has an open neighbourhood $U \subset \mathbb{R}^{2} \times H^{1,1}(X, \mathbb{R})$ such that for all $(\tilde{\alpha}, \tilde{\omega}) \in U$ there exists an extremal pair $(\tilde{\omega}, \tilde{H})$ with coupling constants $\tilde{\alpha}$ and such that $[\tilde{\omega}]=\tilde{\omega}$.

Proof Note that $\eta_{\alpha}(b)=0$, as $b=(\omega, H)$ is a solution of the coupled equations (3.119). Since the map $\mathbf{T}_{\alpha}$ depends linearly on $\alpha=\left(\alpha_{0}, \alpha_{1}\right)$, it can be viewed as a $C^{1}$ map T: $\mathbb{R}^{2} \times \mathcal{W} \rightarrow I_{k, k+2}$, whose the Fréchet derivative at the origin with respect to $\phi$ and $\xi$ is $\delta \mathrm{T}_{\alpha}=\left(\mathrm{Id}-\Pi_{0}\right) \circ \hat{\mathbf{L}}_{\alpha, b}$, by (4.177). Since $H$ is HYM with respect to $\omega$ and $\alpha_{0} \alpha_{1}>0$, Lemma 4.8 applies and $\left(\mathrm{Id}-\Pi_{0}\right) \circ \hat{\mathbf{L}}_{\alpha, b}$ is an isomorphism. Therefore, by the implicit function theorem, there exists an open neighbourhood $U \subset \mathbb{R}^{2} \times \mathcal{H}^{1,1}(X)$ of $(\alpha, \Omega)$ such that for all $(\tilde{\alpha}, \gamma) \in U$ there exists a pair $(\phi, \xi) \in I_{k+4, k+4}$ such that

$$
\mathrm{T}_{\tilde{\alpha}}(\gamma, \phi, \xi) \in \operatorname{ker}\left(\left(\mathrm{Id}-\Pi_{0}\right)\left(\mathrm{Id}-\Pi_{\gamma, \phi, \xi}\right)\right)
$$

so $\mathrm{T}_{\widetilde{\alpha}}(\gamma, \phi, \xi) \in \operatorname{Im} \mathbf{P}_{(\gamma, \phi, \xi)}$ by $(4.175)$. Hence the pair $(\tilde{\omega}, \tilde{H})$ determined by $(\gamma, \phi, \xi)$ is extremal with coupling constant $\tilde{\alpha}$, by Lemma 4.8(1), and smooth by Lemma 4.3. 
Let $H^{1,1}(X, \mathbb{R})^{+} \subset H^{1,1}(X, \mathbb{R})$ denote the "Kähler cone" of $(X, J)$, ie, the open subset of elements $\Omega \in H^{1,1}(X, \mathbb{R})$ such that $\mathcal{K}_{\Omega}$ is non-empty. Given $(\alpha, \Omega) \in$ $\mathbb{R}_{>0}^{2} \times H^{1,1}(X, \mathbb{R})^{+}$, consider the $\alpha$-Futaki character $\mathcal{F}_{\alpha, \Omega}:$ Lie $\Gamma_{I} \rightarrow \mathbb{C}$ defined in (3.108) (or (3.126)). Denote

$$
V(\mathcal{F}):=\left\{(\alpha, \Omega) \mid \mathcal{F}_{\alpha, \Omega}=0\right\} \subset \mathbb{R}_{>0}^{2} \times H^{1,1}(X, \mathbb{R})^{+} .
$$

Theorem 4.11 Let $S$ be the set of pairs $(\alpha, \Omega) \in \mathbb{R}_{>0}^{2} \times H^{1,1}(X, \mathbb{R})^{+}$for which there exists a solution $(\omega, H) \in B_{\Omega}$ to the coupled equations (3.119).

(1) Then $S \cap V(\mathcal{F})$ is open in $V(\mathcal{F})$.

(2) If $\operatorname{Aut}\left(E^{c}, I\right)$ is finite, then $S \subset \mathbb{R}^{2} \times H^{1,1}(X, \mathbb{R})$ is open.

Proof The proof is immediate from Theorem 4.10, together with Proposition 4.2 for part (1) and (4.137) for part (2).

\subsection{Deformations of solutions in the weak coupling limit}

We will now obtain solutions to the coupled equations (3.119) in "weak coupling limit" $0<\left|\alpha_{1} / \alpha_{0}\right| \ll 1$ by deforming solutions $(\omega, H) \in B_{\Omega}$ with coupling constants $\alpha_{0} \neq 0, \alpha_{1}=0$. Since we will study these equations for coupling constants in a small open neighbourhood of a pair $\left(\alpha_{0}, \alpha_{1}\right) \in \mathbb{R}^{2}$ satisfying $\alpha_{0} \neq 0, \alpha_{1}=0$, we can divide the second equation in (3.119) by $\alpha_{0}$. Hence in the sequel we will normalize to $\alpha_{0}=1$ and $\alpha:=\alpha_{1}$ will be called the coupling constant.

Note that for $\alpha=0$, the coupled equations (3.119) are the condition that $\omega$ is a $\operatorname{cscK}$ metric on $(X, J)$ and $H$ is a Hermitian-Yang-Mills reduction of $\left(E^{c}, I\right)$ with respect to $\omega$, so in particular the pair $(\omega, H)$ satisfies the following equations:

$$
\left.\begin{array}{l}
d_{H}^{*} F_{H}=0 \\
L_{\eta_{\omega}} J=0
\end{array}\right\}
$$

Here, $d_{H}^{*} F_{H}=0$ is the Yang-Mills equation, which is equivalent to

$$
d_{H} \Lambda_{\omega} F_{H}=0
$$

by the Kähler identities (see eg [15, Proposition 3]), and $\eta_{S_{\omega}}$ is the Hamiltonian vector field of the scalar curvature $S_{\omega}$ over $(X, \omega)$, so $L_{\eta_{S_{\omega}}} J=0$ is the condition that $\omega$ is an extremal metric on $(X, J)$.

If one attempts to generalize Theorem 4.10 to the weak coupling limit, one observes that Proposition 4.2 cannot be used for $\alpha=0$, but the system of equations (4.178) 
can be viewed as an adiabatic limit of equation (4.132). In fact, a pair $b_{\lambda}:=(\lambda \omega, H)$ satisfies (4.132) with coupling constant $\alpha$, for a real number $\lambda>0$, if and only if

$$
\left.4 \alpha d_{H} \Lambda_{\omega} F_{H}+\lambda^{-1} \eta_{\lambda}\right\lrcorner F_{H}=0, \quad L_{\eta_{\lambda}} J=0,
$$

where $\eta_{\lambda}$ is the Hamiltonian vector field of $S_{\alpha / \lambda}(\omega, H)$ over $(X, \omega)$, and (4.178) is the formal limit of (4.180) when $\lambda \rightarrow \infty$. Hence a strategy to obtain a solution to the coupled equations (3.119) for $0<\left|\alpha_{1} / \alpha_{0}\right| \ll 1$ (equivalently, for $\lambda \gg 0$ ) could be to deform a solution to (3.119) for $\alpha=0$ (which is therefore a solution to (4.178)) to obtain a solution of (4.180). The problem is that the kernel of the operator $\mathbf{L}_{\alpha, b_{\lambda}}$ determined by a solution $b_{\lambda}$ to the coupled equations (3.119) has a discontinuity in the limit $\lambda \rightarrow \infty$. More precisely, this kernel for finite $\lambda>0$ can be identified with Lie $\widetilde{\mathcal{G}}_{I}$ (see Proposition 4.6), whereas the kernel of $\mathbf{L}_{\alpha, b}$ in the limit $\lambda \rightarrow \infty$ is

(4.181) $\left.\left\{(\phi, \xi) \in C^{\infty}(X) \times \Omega^{0}\left(\operatorname{ad} E_{H}\right) \mid \eta_{\phi} \in \operatorname{Lie} \mathcal{H}_{J}, d_{H}^{*}\left(d_{H} \xi+\eta_{\phi}\right\lrcorner F_{H}\right)=0\right\}$

(this follows directly from (4.144)). This discontinuity causes serious technical problems when one attempts to use this strategy within the approach of LeBrun and Simanca.

The source of this difficulty is related to the vanishing of the factor $4 \alpha_{1}$ multiplying the HYM term in the moment maps $\mu_{b}$ when $\alpha_{1}=0$ (see (2.44)). One way to get around this problem is to apply the approach of LeBrun and Simanca to the operator obtained by dropping this factor in the moment map operator $\mathrm{T}_{\alpha}$. Fix an integer $k>n$ and keep the notation of Section 4.3, 4.4. Then the resulting modified moment map operator is

$$
\begin{gathered}
\mathrm{B}_{\alpha}: \mathcal{U} \longrightarrow L_{k}^{2}(X) \times L_{k+2}^{2}\left(\operatorname{ad} E_{H}\right), \\
(\gamma, \phi, \xi) \longmapsto\left(S_{\alpha}(\tilde{b}), \Lambda_{\widetilde{\omega}} F_{H, \tilde{I}}-z_{\widetilde{\omega}}\right),
\end{gathered}
$$

where $\mathcal{U}$ is the open set in (4.156) and $\widetilde{\omega}, \tilde{I}$ and $\tilde{b}$ are given by (4.153) and (4.154).

As we will see below, this modification on the moment map operator within the approach of LeBrun and Simanca produces the following modified extremality condition (cf (4.132)).

Definition 4.12 A pair $b=(\omega, H) \in B_{\Omega}$ is called extremal with coupling constant $\alpha$ in the weak coupling limit if it satisfies the equations

$$
\left.\begin{array}{l}
d_{H}^{*} F_{H}=0, \\
L_{\eta_{\alpha}(b)} J=0,
\end{array}\right\}
$$

where $\eta_{\alpha}(b)$ is the Hamiltonian vector field of $S_{\alpha}(b)$ over $(X, \omega)$. 
Note that the system of equations (4.183) becomes (4.178) when $\alpha=0$, while for arbitrary $\alpha$ any solution to the coupled equations (3.119) is an extremal pair in the weak coupling limit (see (4.179)). To obtain a partial converse, define the characters

$$
\mathcal{F}_{0, \Omega}, \mathcal{F}_{\infty, \Omega}: \text { Lie } \operatorname{Aut}\left(E^{c}, I\right) \longrightarrow \mathbb{C}
$$

as the $\alpha$-Futaki characters of the Kähler class $\Omega$ for $\left(\alpha_{0}, \alpha_{1}\right)$ equal to $(1,0)$ and $(0,1)$ in (3.126), respectively. By (3.126), up to a multiplicative factor, $\left\langle\mathcal{F}_{0, \Omega}, \zeta\right\rangle$ is the Futaki character [22] of the Kähler class $\Omega$ on $(X, J)$ evaluated at $p(\zeta)$, where $p$ is the map in (4.135). It is also clear from (3.126) that the existence of a solution to the coupled equations (3.119) does not necessarily imply the vanishing of $\mathcal{F}_{0, \Omega}$ or $\mathcal{F}_{\infty, \Omega}$.

Proposition 4.13 A solution $b \in B_{\Omega}$ of (4.183) is a solution to the coupled equations (3.119) if $\mathcal{F}_{0, \Omega}=\mathcal{F}_{\infty, \Omega}=0$ and the vector field $\eta_{\alpha}(b)$ over $X$ can be lifted to a holomorphic vector field over the total space of $\left(E^{c}, I\right)$.

Proof By (4.179), $\Lambda_{\omega} F_{H}$ is a vertical holomorphic vector field on the total space of $\left(E^{c}, I\right)$, ie, $\Lambda_{\omega} F_{H} \in \operatorname{Lie} \mathcal{G}_{I}$. Now, if $\mathcal{F}_{\infty, \Omega}=0$, then $H$ is HYM with respect to $\omega$, because in this case, by (3.126) we obtain

$$
\left\|\Lambda_{\omega} F_{H}-z_{\Omega}\right\|_{\omega}^{2}=-\left\langle\mathcal{F}_{\infty, \Omega}, \Lambda_{\omega} F_{H}-z_{\Omega}\right\rangle=0
$$

Moreover, if $\mathcal{F}_{0, \Omega}=\mathcal{F}_{\infty, \Omega}=0$ and $\eta_{\alpha}(b)=p(\zeta)$ for a holomorphic vector field $\zeta$ on $\left(E^{c}, I\right)$, then by a straightforward computation using (3.126), we obtain

$$
\left\|S_{\alpha}(b)-\widehat{S}_{\alpha}\right\|_{\omega}^{2}=\left\langle\mathcal{F}_{0, \Omega}, \eta_{\alpha}(b)\right\rangle+\alpha\left\langle\mathcal{F}_{\infty, \Omega}, \zeta\right\rangle+\alpha\left\langle\theta_{H} \zeta, \Lambda_{\omega} F_{H}-z_{\Omega}\right\rangle=0,
$$

where $\widehat{S}_{\alpha}=\int_{X} S_{\alpha}(b) \omega^{[n]} / \operatorname{Vol}_{\Omega}$, so $b$ is a solution to the coupled equations (3.119).

Extremal pairs in the weak coupling limit enjoy the same good regularity properties:

Lemma 4.14 Let $(\omega, H)$ be a solution of (4.183) such that $\omega$ is a Kähler form of class $C^{2}$ on $(X, J)$ and $H$ is a section of $E^{c} / G$ of class $C^{4}$. Then both $\omega$ and $H$ are smooth.

Proof This follows exactly as Lemma 4.3.

We define now a linear differential operator that is closely related to the linearization of $\mathrm{B}_{\alpha}$ (see (4.182)) when $\alpha=0$ and which will play the role in the weak coupling limit of the Lichnerowicz operator (4.142) in the study of the cscK equation or the operator 
$\mathbf{L}_{\alpha, b}$ defined in Section 4.4 away from the weak coupling limit. This linear differential operator is

$$
\text { C: } \begin{aligned}
L_{k+4}^{2}(X) \times L_{k+4}^{2}\left(\operatorname{ad} E_{H}\right) & \longrightarrow L_{k}^{2}(X) \times L_{k+2}^{2}\left(\operatorname{ad} E_{H}\right), \\
(\phi, \xi) & \left.\longmapsto\left(\mathrm{P}^{*} \mathrm{P} \phi, d_{H}^{*}\left(d_{H} \xi+\eta_{\phi}\right\lrcorner F_{H}\right)\right),
\end{aligned}
$$

where $\mathrm{P}$ is defined as in (4.142). It is easy to see (cf Proposition 4.6) that the operator C is elliptic and self-adjoint with respect to the $L^{2}$-inner product $\langle\cdot, \cdot\rangle_{\omega}$ given by (4.141).

It can be shown as in the proof of Proposition 4.7 that $\mathrm{B}_{\alpha}$ is well-defined and $C^{1}$ and that its Fréchet derivative at the origin $(0,0,0)$ when $\alpha=0$ is given by

$$
\delta \mathrm{B}_{0}(\dot{\gamma}, \dot{\phi}, \dot{\xi})=\mathbf{C}(\dot{\phi}, \dot{\xi})+\left(\left(d S_{\omega}, d \dot{\phi}\right)_{\omega}, 0\right)+\delta_{\dot{\gamma}} \mathrm{B}_{0},
$$

where $\delta_{\dot{\gamma}} \mathrm{B}_{0}$ is the directional derivative of $\mathrm{B}_{0}$ at the origin in the direction $(\dot{\gamma}, 0,0)$ (cf (4.158)).

To proceed as in Section 4.5 following the approach of LeBrun and Simanca, we need to consider the restriction of $\mathrm{B}_{\alpha}$ and $\mathbf{C}$ to suitable subspaces of the Sobolev spaces. Let

$$
L_{k}^{2}(X)^{\mathcal{H}_{J}} \subset L_{k}^{2}(X) \text { and } L_{k}^{2}\left(\operatorname{ad} E_{H}\right)^{\mathcal{G}_{I}} \subset L_{k}^{2}\left(\operatorname{ad} E_{H}\right)
$$

be the closed subspaces consisting of $\mathcal{H}_{J}$-invariant functions and $\mathcal{G}_{I}$-invariant sections, respectively (cf (4.166)) and

$$
\mathcal{V}^{\prime}=\mathcal{U} \cap\left(\mathcal{H}^{1,1}(X) \times L_{k+4}^{2}(X)^{\mathcal{H}_{J}} \times L_{k+4}^{2}\left(\operatorname{ad} E_{H}\right)^{\mathcal{G}_{I}}\right)
$$

(cf (4.167)). By restriction of (4.182) and (4.185), we obtain well-defined maps

(4.187a) $\widehat{\mathrm{B}}_{\alpha}: \mathcal{V}^{\prime} \longrightarrow L_{k}^{2}(X)^{\mathcal{H}_{J}} \times L_{k+2}^{2}\left(\operatorname{ad} E_{H}\right)^{\mathcal{G}_{I}}$,

(4.187b) $\widehat{\mathbf{C}}: L_{k+4}^{2}(X)^{\mathcal{H}_{J}} \times L_{k+4}^{2}\left(\operatorname{ad} E_{H}\right)^{\mathcal{G}_{I}} \longrightarrow L_{k}^{2}(X)^{\mathcal{H}_{J}} \times L_{k+2}^{2}\left(\operatorname{ad} E_{H}\right)^{\mathcal{G}_{I}}$,

where $\widehat{\mathrm{B}}_{\alpha}$ is $C^{1}$ and $\widehat{\mathbf{C}}$ is a linear elliptic operator (cf (4.168)).

Note that in the constructions (4.187) we have used the subspace $L_{k}^{2}\left(\operatorname{ad} E_{H}\right)^{\mathcal{G}_{I}} \subset$ $L_{k}^{2}\left(\operatorname{ad} E_{H}\right)$ rather than the possibly smaller subspace $L_{k}^{2}\left(\operatorname{ad} E_{H}\right)^{\widetilde{\mathcal{G}}_{I}}$, which appeared in (4.168). In practice, we could say that the exact sequence (4.135) in Section 4.4 degenerates to the trivial extension

$$
1 \longrightarrow \mathcal{G}_{I} \longrightarrow \mathcal{H}_{J} \times \mathcal{G}_{I} \longrightarrow \mathcal{H}_{J} \longrightarrow 1
$$

in the weak coupling limit $\alpha \rightarrow 0$. In particular, the centre $\mathfrak{z}\left(\right.$ Lie $\tilde{\mathcal{G}}_{I}$ ) of Lie $\widetilde{\mathcal{G}}_{I}$ (see (4.170)) is now replaced by the centre $\mathfrak{z}\left(\right.$ Lie $\left.\mathcal{H}_{J}\right) \oplus \mathfrak{z}\left(\right.$ Lie $\left.\mathcal{G}_{I}\right)$ of the Lie algebra of 
$\mathcal{H}_{J} \times \mathcal{G}_{I}$ and $\mathbf{P}_{(\gamma, \phi, \xi)}$ (see (4.170)) is replaced by

$$
\begin{aligned}
\mathbf{Q}_{(\gamma, \phi, \xi)}: \mathbb{R} \times \mathfrak{z}\left(\operatorname{Lie} \mathcal{H}_{J}\right) \oplus \mathfrak{z}\left(\operatorname{Lie} \mathcal{G}_{I}\right) & \longrightarrow L_{k+3}^{2}(X)^{\mathcal{H}_{J}} \times L_{k+2}^{2}\left(\operatorname{ad} E_{H}\right)^{\mathcal{G}_{I}}, \\
(t, w, v) & \left.\longmapsto\left(\mathbf{G} d^{*}(w\lrcorner \widetilde{\omega}\right)+t, v\right),
\end{aligned}
$$

with $(\gamma, \phi, \xi) \in \mathcal{V}^{\prime}$.

Lemma 4.15 Let $(\gamma, \phi, \xi) \in \mathcal{V}^{\prime}$. If $\widehat{\mathrm{B}}_{\alpha}(\gamma, \phi, \xi) \in \operatorname{Im} \mathbf{Q}_{(\gamma, \phi, \xi)}$, then $\tilde{b}=(\widetilde{\omega}, \widetilde{H})$ is a solution of (4.183).

Proof This follows exactly as part (2) of Lemma 4.8.

Since $\mathbf{C}$ has kernel (4.181) by elliptic regularity, part (3) of Lemma 4.8 has no direct analogue in the weak coupling limit. Lemma 4.16 will provide a suitable replacement of this part of the lemma. Let $\langle\cdot, \cdot\rangle_{\omega}$ be the $L^{2}$-inner product on $L_{k}^{2}(X)^{\mathcal{H} J} \times$ $L_{k+2}^{2}\left(\operatorname{ad} E_{H}\right)^{\mathcal{G}_{I}}$ given by (4.141). One can prove as in Section 4.4 that the orthogonal projector

$$
\Pi_{(\gamma, \phi, \xi)}^{\prime}: L_{k}^{2}(X)^{\mathcal{H}_{J}} \times L_{k+2}^{2}\left(\operatorname{ad} E_{H}\right)^{\mathcal{G}_{I}} \longrightarrow L_{k}^{2}(X)^{\mathcal{H}_{J}} \times L_{k+2}^{2}\left(\operatorname{ad} E_{H}\right)^{\mathcal{G}_{I}}
$$

onto $\operatorname{Im} \mathbf{Q}_{(\gamma, \phi, \xi)}$ varies smoothly with $(\gamma, \phi, \xi) \in \mathcal{V}^{\prime}$ and, by continuity, there exists an open neighbourhood $\mathcal{V}_{0}^{\prime} \subset \mathcal{V}^{\prime}$ of the origin such that

$$
\operatorname{ker}\left(\operatorname{Id}-\Pi_{(\gamma, \phi, \xi)}^{\prime}\right)=\operatorname{ker}\left(\operatorname{Id}-\Pi_{0}\right) \circ\left(\operatorname{Id}-\Pi_{(\gamma, \phi, \xi)}^{\prime}\right)
$$

for any $(\gamma, \phi, \xi) \in \mathcal{V}_{0}^{\prime}(\operatorname{cf}(4.175))$.

For any pair of non-negative integers $(l, m)$, let $I_{l, m}^{\prime} \subset L_{l}^{2}(X)^{\mathcal{H}_{J}} \times L_{m}^{2}\left(\operatorname{ad} E_{H}\right)^{\mathcal{G}_{I}}$ be the orthogonal complement of $\operatorname{Im} \mathbf{Q}_{0}$. Define

$$
\mathcal{W}^{\prime}=\mathcal{V}_{0}^{\prime} \cap\left(\mathcal{H}^{1,1}(X) \times I_{k+4, k+4}^{\prime}\right) .
$$

Lemma 4.16 The induced map $\widehat{\mathbf{C}}: I_{k+4, k+4}^{\prime} \longrightarrow I_{k, k+2}^{\prime}$ is an isomorphism.

Proof This map is well-defined because $\operatorname{Im} \mathbf{Q}_{0} \subset \operatorname{ker}\left(\mathrm{P} \oplus d_{H}\right)$. If $\widehat{\mathbf{C}}(\phi, \xi)=0$ for some $(\phi, \xi) \in I_{k+4, k+4}^{\prime}$, then $\mathrm{P}^{*} \mathrm{P} \phi=0$, so $\mathrm{P} \phi=0$, which implies $\phi=0$, and $\widehat{\mathbf{C}}(\phi, \xi)=0$ means $d_{H}^{*} d_{H} \xi=0$, so $d_{H} \xi=0$, which implies $\xi=0$. Thus $\widehat{\mathbf{C}}$ is injective. Finally, $\widehat{\mathbf{C}}$ is surjective because so is $\mathrm{P}^{*} \mathrm{P} \oplus d_{H}^{*} d_{H}$.

Define now a LeBrun-Simanca map [38, Section 5]

$$
\begin{aligned}
& \mathbf{B}_{\alpha}: \mathcal{W}^{\prime} \longrightarrow I_{k, k+2}^{\prime}, \\
& (\gamma, \phi, \xi) \longmapsto\left(\mathrm{Id}-\Pi_{0}^{\prime}\right) \circ\left(\mathrm{Id}-\Pi_{(\gamma, \phi, \xi)}^{\prime}\right) \circ \widehat{\mathrm{B}}_{\alpha}(\gamma, \phi, \xi) .
\end{aligned}
$$


As $\mathbf{B}_{\alpha}$ is the composition of $C^{1}$-maps, it is $C^{1}$. Using Lemma 4.16 and [38, Lemma 1], we can see that its directional derivative at the origin in the direction $(0, \dot{\phi}, \dot{\xi})$ for $\alpha=0$ is

$$
\delta_{(\dot{\phi}, \dot{\xi})} \mathbf{B}_{0}=\left(\operatorname{Id}-\Pi_{0}^{\prime}\right) \widehat{\mathbf{C}}(\dot{\phi}, \dot{\xi})=\widehat{\mathbf{C}}(\dot{\phi}, \dot{\xi})
$$

for all $(\dot{\phi}, \dot{\xi}) \in I_{k+4, k+4}^{\prime}$.

We can now prove the two main results of Section 4.5.

Theorem 4.17 Suppose that $\omega$ is an extremal Kähler metric on $(X, J)$ with $\Omega=[\omega]$ and $H$ is a Yang-Mills reduction of $\left(E^{c}, I\right)$ with respect to $\omega$. Then $(0, \Omega)$ has an open neighbourhood $U \subset \mathbb{R} \times H^{1,1}(X, \mathbb{R})$ such that for all $(\widetilde{\alpha}, \widetilde{\omega}) \in U$ there exists an extremal pair $(\widetilde{\omega}, \widetilde{H})$ with coupling constant $\widetilde{\alpha}$ in the weak coupling limit such that $[\tilde{\omega}]=\tilde{\omega}$.

Proof This follows as Theorem 4.10, combining (4.191) with Lemma 4.16 and the implicit function theorem, and then using Lemmas 4.15 and 4.14 .

In the following theorem, we say that a reduction $H \in \mathcal{R}$ is irreducible if its Chern connection is irreducible, that is, if its isotropy group inside the gauge group $\mathcal{G}_{H}$ of $E_{H}$ is minimal; the centre of $G$ (see Section 2.3 and also [20, Section 4.2.2]).

Theorem 4.18 Assume that there is a cscK metric $\omega$ on $(X, J)$ with cohomology class $\Omega$ and there are no non-zero Hamiltonian Killing vector fields on $X$. Then:

(1) If $\left(E^{c}, I\right)$ admits an irreducible HYM reduction $H$ with respect to $\omega$, then $(0, \Omega)$ has an open neighbourhood $U \subset \mathbb{R} \times H^{1,1}(X, \mathbb{R})$ such that for all $\left(\widetilde{\alpha}_{1}, \widetilde{\omega}\right) \in U$, there exists a solution $(\widetilde{\omega}, \widetilde{H})$ to the coupled equations (3.119) with coupling constant $\widetilde{\alpha}=\left(1, \widetilde{\alpha}_{1}\right)$ and $\widetilde{\omega} \in \widetilde{\omega}$.

(2) If $\left(E^{c}, I\right)$ admits a $H Y M$ reduction $H$ with respect to $\omega$, then there exists $\epsilon>0$ such that for all $\widetilde{\alpha}_{1} \in \mathbb{R}$ with $-\epsilon<\widetilde{\alpha}_{1}<\epsilon$, there exists a solution $(\widetilde{\omega}, \tilde{H})$ to the coupled equations (3.119) with coupling constants $\left(1, \widetilde{\alpha}_{1}\right)$ and $\widetilde{\omega} \in \widetilde{\omega}$.

Proof Since HYM reductions are Yang-Mills, Theorem 4.17 implies that for all $(\widetilde{\alpha}, \widetilde{\omega})$ in a neighbourhood $U \subset \mathbb{R} \times H^{1,1}(X, \mathbb{R})$ of $(0, \Omega)$, there exists an extremal pair $(\widetilde{\omega}, \widetilde{H})$ with coupling constant $\widetilde{\alpha}$ in the weak coupling limit with $[\widetilde{\omega}]=\widetilde{\omega}$ and $\widetilde{H}$ irreducible.

Part (1) follows now since the function $S_{\alpha}(\tilde{\omega}, \tilde{H})$ defined by (4.133) is constant on $X$ for any extremal pair $(\widetilde{\omega}, \tilde{H})$, as Lie $\mathcal{H}_{J}=0$ and, furthermore, the vertical realholomorphic vector field on $\left(E^{c}, I\right)$ defined by $\Lambda_{\widetilde{\omega}} F_{\widetilde{H}}$ is in $\mathfrak{z}$, as $\widetilde{H}$ is irreducible.

Part (2) follows from Theorem 4.17 and Proposition 4.13, because $\mathcal{F}_{0, \Omega}=\mathcal{F}_{\infty, \Omega}=0$ by (3.126), as Lie $\mathcal{H}_{J}=0$ and $\left(E^{c}, I\right)$ admits a HYM reduction $H$ with respect to $\omega$. 


\section{Examples and cscK metrics on ruled manifolds}

This section contains some examples of solutions to the coupled equations (0.2). In Section 5.4 we also discuss how the existence of solutions in the limit case $\alpha_{0}=0$ can be applied, using results of Y J Hong in [30], to obtain cscK metrics on ruled manifolds.

\subsection{Projectively flat bundles}

Let $\left(E^{c}, I\right)$ be a holomorphic principal $G^{c}$-bundle over a compact complex manifold $X$. We fix a maximal compact subgroup $G \subset G^{c}$ and a $G$-invariant metric $(\cdot, \cdot)$ on $\mathfrak{g}$. Suppose that there exists a $G$-reduction $H$ on $E^{c}$ and a Kähler metric $\omega$ on $X$ satisfying

$$
\left.\begin{array}{l}
F_{H}=z \omega, \\
S_{\omega}=\widehat{S},
\end{array}\right\}
$$

where $F_{H}$ is the curvature of the Chern connection of $H, z$ is the element of $\mathfrak{z}$ (see (1.15)) given by (1.18) and $\widehat{S} \in \mathbb{R}$. It is then straightforward that the pair $(\omega, H)$ provides a solution of the coupled equations (0.2). Note that the first equation in (5.192) implies that the $G$-bundle $E_{H}$ corresponding to $H$ is projectively flat, ie, it is given by a representation $\pi_{1}(X) \rightarrow G / Z(G)$, where $Z(G)$ denotes the centre of $G$. Moreover, it implies the following topological constraint

$$
\left[z \wedge F_{H}\right]=|z|^{2}[\omega] \in H^{2}(X, \mathbb{R}),
$$

where $\left[F_{H} \wedge z\right]$ is the Chern-Weil class associated to the $G$-invariant linear form $(\cdot, z)$ on $\mathfrak{g}$. We discuss now some examples of solutions of (5.192). We apply Theorem 4.18(1) to perturb the Kähler class of the given solution in order to obtain new solutions that do not satisfy the topological constraint (5.193).

Example 5.1 Let $X$ be a compact Riemann surface. Then the coupled equations (0.2), for a $G$-reduction $H$ on $E^{c}$ and a Kähler metric $\omega$ on $X$, split into the system in separated variables (5.192), since $\operatorname{dim}_{\mathbb{C}} X=1$ and the term $\left(F_{H} \wedge F_{H}\right)$ vanishes. Then the solutions of the coupled equations $(0.2)$ are given by pairs $(\omega, H)$, where $\omega$ is a cscK metric and $H$ is a $G$-reduction such that its Chern connection is Hermitian-YangMills (1.17). Due to the Narasimhan and Seshadri theorem [14], and Ramanathan's generalization [47], examples of solutions of the coupled equations $(0.2)$ are given by polystable $G^{c}$-bundles over $X$.

Remark 5.2 In [46], Pandharipande used geometric invariant theory to compactify the moduli space of pairs $(X, F)$ consisting of a smooth algebraic curve $X$ of genus $g>1$, polarised by a multiple of its canonical bundle, and a semistable vector bundle 
$F$ over the curve. By [46, Proposition 8.2.1], such a pair is GIT stable if and only if $E$ is Mumford stable. An interesting issue is that this decoupling phenomenon for the stability condition of a pair $(X, F)$ is reflected in the decoupling of the equations (0.2), as already observed in Example 5.1. In fact, combining the Narasimhan-Seshadri theorem with the uniformization theorem on Riemann surfaces, it follows that any GIT stable pair $(X, F)$ in Pandharipande's construction, with $X$ smooth, admits one and only one (irreducible) solution of (0.2) with Kähler class equal to the class of the polarisation. This gives some evidence to the claim that a Hitchin-Kobayashi correspondence for equations (0.2) exists in arbitrary dimensions, as conjectured by the authors [25]. An important difference with the curve case is that of course in higher dimensions one expects that the stability condition equivalent to the existence of solutions will involve conditions on the base manifold as well. In [25], a new notion of stability for degree zero bundles and polarised varieties has been defined. We hope to address the relation of this stability condition and the existence of solutions of $(0.2)$ in future work.

Let $(X, L)$ be a compact polarised manifold of complex dimension $n$. Suppose that there exists a cscK metric

$$
\omega=\frac{\mathbf{i}}{2 \pi} F_{H} \in c_{1}(L),
$$

where $F_{H}$ is the curvature of a Hermitian metric $H$ on $L$. Then $(\omega, H)$ is a solution of (5.192), and hence a solution of (0.2). Since $H$ is trivially an irreducible HYM metric with respect to $\omega$, if there are no non-zero Hamiltonian holomorphic vector fields on $X$, we can apply Theorem 4.18(1) obtaining solutions of $(0.2)$ with non-zero ratio of the coupling constants and Kähler class close to $[\omega]$ in $H^{1,1}(X, \mathbb{R})$.

Example 5.3 Let $X$ be a degree four hypersurface of $\mathbb{P}^{3}$ and set $L=\mathcal{O}_{X}(1)$. Then $X$ is a K3 surface and, by Yau's solution [61] of the Calabi conjecture (see eg [6]), there exists a unique Kähler Ricci flat metric $\omega \in c_{1}(L)$. Since $(X, \omega)$ is Kähler Ricci flat, any holomorphic vector field on $X$ is $\omega$-parallel and so Lie(Aut $X$ ) contains no non-zero Hamiltonian holomorphic vector fields. Therefore, applying Theorem 4.18(1), we obtain solutions of (0.2) with non-zero ratio of the coupling constants $\alpha_{1} / \alpha_{0}$ and Kähler class $\widetilde{\omega}$ close to $\Omega=[\omega]$ in $H^{1,1}(X, \mathbb{R})$. As the dimension of $H^{1,1}(X, \mathbb{R})$ is 20 , we can assume that $\widetilde{\omega}$ is not contained in the real line spanned by $\Omega$, and so it is not obvious a priori that such a Kähler class contains a solution of $(0.2)$ for our choice of manifold $X$ and bundle $L$.

When $(X, \omega)$ is a flat Kähler torus, we can relax condition (5.192) and assume that $E$ is an arbitrary projectively flat Hermitian bundle over $X$. 
Example 5.4 Let $X \cong \mathbb{C}^{n} / \Lambda_{X}$ be a complex torus given by a lattice $\Lambda_{X}$ in $\mathbb{Z}^{2 n}$ and endowed with a flat Kähler metric $\omega$. Examples of holomorphic vector bundles $E$ over $X$ admitting a projectively flat Hermitian metric $H$ are given by representations of a central extension of $\Lambda_{X}$ into $U(r) \subset \mathrm{GL}(r, \mathbb{C})$. Suppose that $E$ is given by an irreducible representation of $\Lambda_{X}$ and take a projectively flat Hermitian metric $H$ on $E$, with curvature $\tau$ Id. By a conformal change on $H$, we can assume that $\tau$ is harmonic, and hence it is constant with respect to the natural coordinates in the torus. Then $(\omega, H)$ is a solution to the coupled equations (0.2) for arbitrary value of the coupling constants $\alpha_{0}$ and $\alpha_{1}$.

Remark 5.5 In [50], Schumacher and Toma constructed a moduli space of (nonuniruled) polarised Kähler manifolds equipped with stable vector bundles, using versal deformations. This moduli space is endowed with a Kähler metric, provided that the cohomological constraint (5.193) is satisfied, the base manifold $X$ is Kähler-Einstein and the bundle is projectively flat. The gauge-theoretic equations corresponding to this moduli construction are therefore equivalent to (5.192), whose solutions are in particular solutions to the coupled equations (0.2). Note here that the cscK equation and the Kähler-Einstein equation are equivalent, by Hodge theory, if the class of the polarisation is a multiple of $c_{1}(X)$.

In the examples of Section 5.1, the coupled equations (0.2) admit decoupled solutions arising from the system in separated variables (5.192). There is a geometric interpretation for this in terms of the extended gauge group $\widetilde{\mathcal{G}}$ in (1.20) associated to a solution $(\omega, H)$ to $F_{H}=z \omega$ and the moment map interpretation of (0.2) in Section 2. Namely, the Chern connection $A$ of $H$ determines a Lie algebra splitting of the short exact sequence

$$
0 \longrightarrow \text { Lie } \mathcal{G} \longrightarrow \text { Lie } \tilde{\mathcal{G}} \longrightarrow \text { Lie } \mathcal{H} \longrightarrow 0
$$

(see (1.32)). The splitting is given by the Lie algebra homomorphism

$$
\Phi: \text { Lie } \mathcal{H} \cong C_{0}^{\infty}(X) \longrightarrow \operatorname{Lie} \tilde{\mathcal{G}}
$$

$$
\phi \longmapsto \theta_{A}^{\perp} \eta_{\phi}-\phi z
$$

(see (1.34)), where $\left.\eta_{\phi}\right\lrcorner \omega=d \phi$ and $\theta_{A}^{\perp}$ is the horizontal lift with respect to the connection $A$. To see this, note that

$$
\begin{aligned}
{\left[\Phi\left(\phi_{1}\right), \Phi\left(\phi_{2}\right)\right] } & =\left[\theta_{A}^{\perp} \eta_{\phi_{1}}-\phi_{1} z, \theta_{A}^{\perp} \eta_{\phi_{2}}-\phi_{2} z\right] \\
& =\theta_{A}^{\perp}\left[\eta_{\phi_{1}}, \eta_{\phi_{2}}\right]-\left\{\phi_{1}, \phi_{2}\right\} z+\left(F_{A}-z \omega\right)\left(\eta_{\phi_{1}}, \eta_{\phi_{2}}\right) \\
& =\Phi\left(\left\{\phi_{1}, \phi_{2}\right\}\right)+\left(F_{A}-\omega z\right)\left(\eta_{\phi_{1}}, \eta_{\phi_{2}}\right),
\end{aligned}
$$


where $\left\{\phi_{1}, \phi_{2}\right\}$ is the Poisson bracket in $C_{0}^{\infty}(X)$ given by $\omega$. Note that this homomorphism does not extend in general to the Lie algebra of the group of diffeomorphisms of $X$. Therefore, when $\operatorname{dim}_{\mathbb{C}} X=1$ or $E$ is projectively flat, the coupled system (0.2) may have "decoupled" solutions due to the fact that Lie $\widetilde{\mathcal{G}}$ is a semidirect product of Lie $\mathcal{G}$ and Lie $\mathcal{H}$.

\subsection{Homogeneous bundles over homogeneous Kähler manifolds}

For the basic material on this topic we refer to Besse [6] and Kobayashi [34]. Let $X$ be a compact homogeneous Kählerian manifold (ie, admitting a Kähler metric) of a compact group $G$. In other words, $X=G / G_{o}$, for a closed subgroup $G_{o} \subset G$, equipped with the canonical $G$-invariant complex structure (see [6, Remark 8.99]). Then homogeneous holomorphic vector bundles $E$ of rank $r$ over $X$ are in one-to-one correspondence with representations of $G_{o}$ in $\operatorname{GL}(r, \mathbb{C})$. For any invariant Kähler metric $\omega$ on $X$, there exists a unique $G$-invariant Hermitian-Yang-Mills unitary connection $A$, provided that the representation inducing $E$ is irreducible (see [34, Proposition 6.1]). Moreover, for any such choice of invariant metric and connection, the scalar curvature $S_{\omega}$ and the function $\Lambda_{\omega}^{2} \operatorname{tr}\left(F_{A} \wedge F_{A}\right)$ on $X$ are $G$-invariant and hence constant. It hence turns out that $A$ satisfies the system of equations

$$
\left.\begin{array}{l}
\Lambda_{\omega} F_{A}=\mathbf{i} \lambda \mathrm{Id}, \\
\Lambda_{\omega}^{2} \operatorname{tr}\left(F_{A} \wedge F_{A}\right)=-\frac{4 \widehat{c}}{(n-1) !},
\end{array}\right\}
$$

where $\hat{c} \in \mathbb{R}$ is as in (2.51) and $\lambda \in \mathbb{R}$ is determined by the first Chern class of $E$ and $[\omega]$. Equations (5.195) corresponds to the limit

$$
\alpha_{0} \rightarrow 0
$$

in (0.2). Fix a pair of arbitrary coupling constants $\alpha_{0}, \alpha_{1}>0$ and a homogeneous holomorphic vector bundle $E$ over $X$ associated to an irreducible representation. Then any Kähler class on $X$ determines a unique $G$-invariant solution $(\omega, A)$ to the coupled equations with coupling constants $\alpha_{0}$ and $\alpha_{1}$. To see this, note that each de Rham class on $X$ (in particular, each Kähler class) contains a unique $G$-invariant representative, obtained from an arbitrary representative by averaging. Trivially, the scalar curvature of any $G$-invariant Kähler metric is constant. Therefore, the unique $G$-invariant solution of (0.2) arises as a simultaneous solution of the cscK equation and (5.195), corresponding to the limit cases $\alpha_{0}=0$, and $\alpha_{1}=0$.

Example 5.6 Let $(X, \omega)$ be a compact homogeneous Kähler-Einstein surface $G / G_{0}$. By [6, Corollary 8.98], this means that $X$ is a complex torus or it is simply connected. 
Let $E$ be a homogeneous vector bundle on $X$ induced by an irreducible representation of $G_{o}$ in $\mathrm{SU}(r)$, with induced $G$-invariant Hermitian metric $H$ and $G$-invariant unitary connection $A$. Then the pair $(\omega, A)$ satisfies the system of equations

$$
\left.\begin{array}{l}
F_{A}^{+}=0, \\
\alpha_{0}\left(\rho_{\omega}-c^{\prime} \omega\right)=\alpha_{1}\left(2\left(\Lambda_{\omega} F_{A}\right) \wedge F_{A}-\Lambda_{\omega}\left(F_{A} \wedge F_{A}\right)-c^{\prime \prime} \omega\right),
\end{array}\right\}
$$

for real numbers $c^{\prime}, c^{\prime \prime}$, where $\rho_{\omega}$ is the Ricci form of $\omega$ and $F_{A}^{+}=0$ is the AntiSelf-Duality equation for the connection $A$. To prove this, note that $A$ is HYM and

$$
\begin{aligned}
2\left(\Lambda_{\omega} F_{A}\right) \wedge F_{A}-\Lambda_{\omega}\left(F_{A} \wedge F_{A}\right) & =-\Lambda_{\omega}\left(F_{A} \wedge F_{A}\right) \\
& =\Lambda_{\omega}\left(\left|F_{A}\right|^{2} \omega^{2}\right) \\
& =\left|F_{A}\right|^{2} \omega,
\end{aligned}
$$

(see (2.48)), where $|\cdot|$ is the pointwise norm with respect to $\omega$. Hence $(\omega, A)$ satisfies (5.196) because the function $\left|F_{A}\right|^{2}$ is constant over $X$ by invariance. Observe that the system (5.196) is stronger than (0.2). Indeed, it can be readily checked from [6, Proposition 9.61] that if $(\omega, A)$ satisfies (5.196), then the associated invariant Riemannian metric on the total space of the frame $U(r)$-bundle of $(E, H)$ over $X$, constructed as in Section 2.3, is Einstein, and therefore $(\omega, A)$ satisfies (0.2), by (2.60).

\subsection{Stable bundles and cscK manifolds}

We supply now some cases where Theorem 4.18 can be applied, obtaining examples of solutions with non-zero ratio of the coupling constants and fixed Kähler class. Starting with a $\operatorname{cscK}$ metric, we check that the new Kähler metrics that we obtain are not cscK. Using the contents of Section 3.4, we also give an explicit Example 5.9 in which there cannot exist solutions to the coupled equations.

Example 5.7 Let $X$ be a high degree hypersurface of $\mathbb{P}^{3}$. By theorems of Aubin and Yau (see eg [6, Theorem 11.7]), there exists a unique Kähler-Einstein metric $\omega \in c_{1}(X)$ with negative (constant) scalar curvature. Moreover, $c_{1}(X)<0$ implies that the group of automorphisms of the complex manifold $X$ is discrete (see [6, Proposition 2.138]). Let $E$ be a smooth SU(2)-principal bundle over $X$ with second Chern number

$$
k=\frac{1}{8 \pi^{2}} \int_{X} \operatorname{tr}\left(F_{A} \wedge F_{A}\right) \in \mathbb{Z},
$$

where $A$ is a connection on $E$. When $k$ is sufficiently large, the moduli space $M_{k}$ of anti-self-dual (ASD) connections $A$ on $E$ with respect to $\omega$ is non-empty (see [20, Section 10.1.14]). Moreover, if $k$ is large enough, $M_{k}$ is non-compact but admits a 
compactification. Let $A$ be a connection that determines a point in $M_{k}$. Then $A$ is irreducible and so we can apply Theorem 4.18(1), obtaining solutions $\left(\omega_{\alpha}, A_{\alpha}\right)$ of (0.2) with $\left[\omega_{\alpha}\right]=[\omega]$, nonzero values of the coupling constants $\alpha_{0}, \alpha_{1}$, and small ratio

$$
\alpha=\frac{\alpha_{1}}{\alpha_{0}} .
$$

We claim that if the pointwise norm

$$
\left|F_{A_{0}}\right|_{\omega_{0}}^{2}: X \longrightarrow \mathbb{R}
$$

of the initial HYM connection $A_{0}=A$ with respect to the Kähler-Einstein metric $\omega_{0}=\omega$ is not constant, then $\omega_{\alpha}$ is not $\operatorname{cscK}$ for $0<\alpha \ll 1$. To see this, note that $\left(\omega_{\alpha}, A_{\alpha}\right)$ approaches uniformly to $\left(\omega_{0}, A_{0}\right)$ as $\alpha \rightarrow 0$ (see Theorem 4.17 ) and so

$$
\left.\lim _{\alpha \rightarrow 0}|| F_{A_{\alpha}}\right|_{\omega_{\alpha}} ^{2}-\left.\left|F_{A_{0}}\right|_{\omega_{0}}^{2}\right|_{L^{\infty}}=0
$$

Hence if (5.197) is not constant, then $\left|F_{A_{\alpha}}\right|_{\omega_{\alpha}}^{2}$ is not constant for small $\alpha$, so the claim follows from

$$
S_{\omega_{\alpha}}=\frac{c}{\alpha_{0}}-\alpha \Lambda_{\omega_{\alpha}}^{2}\left(F_{A_{\alpha}} \wedge F_{A_{\alpha}}\right)=\frac{c}{\alpha_{0}}+\alpha\left|F_{A_{\alpha}}\right|_{\omega_{\alpha}}^{2}
$$

where $c \in \mathbb{R}$. This last equation is satisfied because $\left(\omega_{\alpha}, A_{\alpha}\right)$ is a solution to (0.2). To choose an ASD connection for which (5.197) is not a constant, we consider a sequence of ASD connections $\left\{A^{l}\right\}_{l=0}^{\infty}$ defining points of $M_{k}$ and approaching a point on the boundary of the compactification. When $l \gg 0$, the connections $A_{l}$ start bubbling. This bubbling is reflected in the fact that the function (5.197) becomes more and more concentrated in a finite number of points of the manifold. Therefore, eventually, we obtain an ASD irreducible connection for which (5.197) is not a constant.

To be more precise, recall that any point on the boundary of the compactification of $M_{k}$ is given by an ideal connection (see [20, Definition 4.4.1]), ie, an unordered $d$-tuple $\left(p_{1}, \ldots, p_{d}\right)$ of points on $X$ and a connection $A_{\infty}$ on $M_{k-d}$, the moduli space of ASD connections on a suitable smooth $\mathrm{SU}(2)$-bundle $E_{k-d}$ with second Chern number $k-d$. If $\left[A_{l}\right] \rightarrow\left[A_{\infty}\right]$ as $l \rightarrow \infty$, then for any continuous function $f$ on $X$ (see [20, Theorem 4.4.4]),

$$
\lim _{l \rightarrow \infty} \int_{X} f \operatorname{tr}\left(F_{A_{l}} \wedge F_{A_{l}}\right)=\int_{X} f \operatorname{tr}\left(F_{A_{\infty}} \wedge F_{A_{\infty}}\right)+8 \pi^{2} \sum_{m=1}^{d} f\left(p_{m}\right) .
$$

Take $A_{\infty}$ in $M_{k-d}$ with $d>0$. If $\left|F_{A_{l}}\right|_{\omega}^{2}$ is constant for all $l$, using (5.198) and the equality

$$
\left|F_{A_{l}}\right|_{\omega}^{2} \omega^{2}=\operatorname{tr}\left(F_{A_{l}} \wedge F_{A_{l}}\right)
$$


we obtain that $d=0$ and hence a contradiction (eg, in (5.198), take a sequence $\left\{f_{j}\right\}_{j=1}^{\infty}$ of test functions approaching the delta function of a point $p_{i}$ on $X$ ).

The hypothesis of Theorem 4.18 hold in much more generality. By the DonaldsonUhlenbeck-Yau Theorem (Donaldson [16], and Uhlenbeck and Yau [58]), which admits a generalization to principal bundles (see $[3 ; 48]$ ), a family of examples generalizing Example 5.7 is provided by polystable holomorphic principal bundles over cscK manifolds with no non-zero Hamiltonian Killing vector fields. Recall that this theorem states that if a holomorphic principal $G^{c}$-bundle $\left(E^{c}, I\right)$ is (Mumford-Takemoto) polystable with respect to a Kähler class $\Omega$ on a compact complex manifold $X$, then for any Kähler form $\omega \in \Omega$ there exists a reduction $H$ of $\left(E^{c}, I\right)$ to $G$ that is HYM with respect to $\omega$.

Let $(X, L)$ be a compact polarised manifold whose first Chern class $c_{1}(X)$ satisfies

$$
c_{1}(X)=\lambda c_{1}(L)
$$

for some $\lambda \in \mathbb{Z}$. When $\lambda<0$ (eg if $X$ is a high degree hypersurface of $\mathbb{P}^{m}$ ), $X$ has finite group of automorphisms and by the above result of Aubin and Yau, there exists a unique Kähler-Einstein metric $\omega \in c_{1}(L)$. If $\lambda=0$, then by Yau's a solution to Calabi's conjecture (see eg [6, Theorem 11.7]), there exists a unique Ricci flat metric on $c_{1}(L)$. As the dimension of the group of automorphisms of such manifolds is equal to its first Betti number (see [6, Remark 11.22]), the simply connected ones (eg K3 surfaces) are complex Ricci flat manifolds with finite group of automorphisms. If $\lambda>0$, it has been recently proved (Chen, Donaldson and Sun [12], and Tian [55]) that $c_{1}(L)$ admits a Kähler-Einstein metric if and only if $(X, L)$ is $\mathrm{K}$-stable. Let us restrict to the case

$$
X=\mathbb{P}^{2} \sharp m \overline{\mathbb{P}}^{2},
$$

the complex surface obtained by blowing up $\mathbb{P}^{2}$ at $m$ generic points (see Tian and Yau [57]). If we take $m$ such that $3<m<8$ then $c_{1}(X)>0, X$ has finite automorphism group (see Tian [56, Remark 3.12]) and it was proved in [57] that $X$ admits a KählerEinstein metric.

On the other hand, given a polarised projective manifold $(X, L)$ (without any assumption on $\left.c_{1}(X)\right)$, an asymptotic result of Maruyama [44] states that there exist $c_{1}(L)$-stable vector bundles $E$ over $X$ of rank $r$, provided that $r>\operatorname{dim} X>2$ and

$$
c_{2}(E) \cdot c_{1}(L)^{n-2} \gg 0 .
$$

If $X$ has finite group of automorphisms and it is endowed with a Kähler-Einstein metric $\omega \in c_{1}(L)$ as before, then we can apply Theorem 4.18. 
Example 5.8 Let $(X, \omega)$ be a Kähler-Einstein manifold. Then $\omega$ is a cscK metric, which determines a Hermitian-Yang-Mills metric $H$ on the tangent bundle $E^{c}=T X$. The pair $(\omega, H)$ is a solution to (3.119) with $\alpha_{1}=0$, but it is not a solution with $\alpha_{1} \neq 0$ unless the Chern connection of $H$ is flat. If $c_{1}(X) \leq 0$, then there are no non-zero Hamiltonian holomorphic vector fields over $X$, so $\mathcal{F}_{0, \Omega}=\mathcal{F}_{\infty, \Omega}=0$ and as in Theorem 4.18, $(0, \Omega)$ has an open neighbourhood $U \subset \mathbb{R} \times H^{1,1}(X, \mathbb{R})$ such that for all $(\widetilde{\alpha}, \widetilde{\omega}) \in U$, there exists a solution $(\widetilde{\omega}, \tilde{H})$ to the coupled equations (3.119) with coupling constants satisfying $\alpha_{1} / \alpha_{0}=\tilde{\alpha}$ and $[\tilde{\omega}]=\widetilde{\omega}$.

We will now construct an example where the $\alpha$-Futaki character $\mathcal{F}_{I}$ obstructs the existence of solutions to the coupled equations for small ratio of the coupling constants.

Example 5.9 Let $(X, \omega)$ be a Kähler manifold such that $\omega$ is not a $\operatorname{cscK}$ metric but it is extremal (eg $\mathbb{C P}^{2}$ blown up at one point [8]). Recall from Section 4.1 that the extremality condition is equivalent to the condition that $S_{\omega}$ is the Hamiltonian function of a real holomorphic Killing vector field $\eta$. Since $\omega$ is not a cscK metric, it follows from (3.126) and (4.184) that the classical Futaki character of the Kähler class $\Omega=[\omega]$ evaluated at $\eta$ is

$$
\left\langle\mathcal{F}_{0, \Omega}, \eta\right\rangle=\int_{X}\left(S_{\omega}-\widehat{S}\right)^{2} \omega^{[n]}>0 .
$$

Note that $\eta$ lifts to a holomorphic vector field $\zeta \in \operatorname{Lie} \operatorname{Aut}(T X)$ on the holomorphic tangent bundle $E^{c}=T X$ of $X$. It follows from (3.126) that the $\alpha$-Futaki character $\mathcal{F}_{I}$ evaluated at $\zeta$ is positive for sufficiently small values of $\alpha_{1} / \alpha_{0}>0$. Hence the pair $(X, T X)$ does not admit a solution $(\omega, H)$ to (3.119) with $\omega \in \Omega$ and these values of the coupling constants.

Given an arbitrary holomorphic principal $G^{c}$-bundle $E^{c}$ over $X$, the obstruction to lift a holomorphic vector field on $X$ to a $G^{c}$-invariant holomorphic vector field on $E^{c}$ lies in $H^{1}\left(X, \operatorname{ad} E^{c}\right)(\mathrm{cf}(1.30))$. Note that when $G^{c}=\mathbb{C}^{*}$, the previous argument always applies.

Let $E^{c}$ be a stable holomorphic principal $G^{c}$-bundle over a polarised manifold $(X, L)$. In this situation, the Donaldson-Uhlenbeck-Yau theorem allows us to think of the coupled equations as a generalization of the constant scalar curvature equation for a Kähler metric $\omega \in c_{1}(L)$. More precisely, given such $\omega$ there exists a unique HYM reduction $H$ on $E^{c}$ with respect to $\omega$ and therefore (3.119) can be interpreted as a single scalar equation for the Kähler metric. Although this approach may not be very useful in general, it becomes very explicit for the case of a line bundle $E^{c}$. In this 
case, a solution of the coupled equations is equivalent to a pair $(\omega, \beta)$, where $\beta$ is a harmonic $(1,1)$-form with $[\beta] / \sqrt{2}=2 \pi c_{1}\left(E^{c}\right)$ and satisfying

$$
S_{\omega}-\alpha|\beta|_{\omega}^{2}=c^{\prime}
$$

for a real constant $c^{\prime} \in \mathbb{R}$, where $\alpha=\alpha_{1} / \alpha_{0}$. As for this, we simply note that $H$ is a HYM Hermitian metric on $E^{c}$ with respect to $\omega$ if and only if $i F_{H}$ is harmonic. Therefore, for line bundles, the coupled equations provide a deformation of the constant scalar curvature equation by a harmonic $(1,1)$-form (cf [53]). This point of view has been recently used by Keller and Tønnesen-Friedman to find solutions of the coupled equations on polarised complex 3-folds that do not admit any cscK metric [32]. We should stress that in general equation (5.200) is as difficult as the cscK equation, which has been completely solved only in the Kähler-Einstein case [12; 55]. It would be interesting to study these deformations in terms of $\mathrm{K}$-stability.

\section{4 cscK metrics on ruled manifolds}

We now briefly discuss the relation between equation (5.195), given by the limit

$$
\alpha_{0} \rightarrow 0
$$

in (0.2), and the existence of solutions to the $\operatorname{cscK}$ equation on ruled manifolds. We will use existence results of Y J Hong [29; 30].

Let $(X, J, \omega)$ be a compact Kähler manifold with constant scalar curvature and $E$ a holomorphic stable vector bundle of degree zero over $X$ (examples of this type were already provided in Section 5.3). Let $H$ be a Hermitian metric on $E$ whose Chern connection $A$ is HYM (it exists by the Donaldson-Uhlenbeck-Yau theorem $[16 ; 58]$ ). Let $L$ be the tautological bundle over the projectivised bundle $\mathbb{P}(E)$ of $E$ and $F_{\mathrm{A}_{L^{*}}}$ the curvature of the connection induced by $A$ on $L^{*}$. Then the 2 -form

$$
\frac{i}{2 \pi} F_{\mathrm{A}_{L^{*}}}
$$

is non-degenerate on the fibres and in fact it induces the Fubini-Study metric, so

$$
\widehat{\omega}_{k}=\frac{i}{2 \pi} F_{\mathrm{A}_{L^{*}}}+k \pi^{*} \omega
$$

is a Kähler metric on $\mathbb{P}(E)$ for $k$ large enough. When the automorphism group of $(X, J)$ is finite, Y J Hong [29] used a deformation argument to prove that the cohomology class $\left[\hat{\omega}_{k}\right]$ contains a cscK metric for $k \gg 0$. Let $\widetilde{\mathcal{G}}$ be the extended gauge group of the frame $\mathrm{PU}(r)$-bundle of the Hermitian vector bundle $(E, H)$ and $\widetilde{\mathcal{G}}_{I} \subset \widetilde{\mathcal{G}}$ 
the stabilizer of the connection $A$. The assumption on Aut $X$ was removed in [30] (see [30, Definition I.A]), under the additional conditions that the subgroup

$$
\widetilde{\mathcal{G}}_{I} \subset \operatorname{Aut} \mathbb{P}(E)
$$

is finite and:

$$
\Lambda_{\omega}^{2}\left(\operatorname{tr} F_{A} \wedge \operatorname{tr} F_{A}+\operatorname{tr} F_{A} \wedge \rho_{\omega}+F_{A} \wedge F_{A}\right)=\text { const. }
$$

Since $c_{1}(E)=0$, this second condition reduces to

$$
\Lambda_{\omega}^{2} \operatorname{tr}\left(F_{A} \wedge F_{A}\right)=-\frac{4 \widehat{c}}{(n-1) !} \in \mathbb{R} .
$$

The condition (5.201) appears when one splits the linearization of the cscK equation on $\mathbb{P}(E)$ into vertical and horizontal parts with respect to the connection $A$.

Hence we conclude that when $c_{1}(E)=0$ and $\mathcal{G}_{I}$ is finite, the existence of a solution to (5.195) is a sufficient condition for the existence of a cscK metric in the cohomology class $\left[\widehat{\omega}_{k}\right]$ for $k \gg 0$ (see [30, Theorem III.A]). It would be interesting to study further this relation, trying to prove that the existence of solutions to the coupled equations for small $\alpha_{1} / \alpha_{0}>0$ implies the existence of constant scalar curvature Kähler metrics on $\mathbb{P}(E)$ with Kähler class $k c_{1}(L)$ for large $k$. This would provide a generalization of Hong's results in [30].

\section{References}

[1] M C Abbati, R Cirelli, A Manià, P Michor, The Lie group of automorphisms of a principal bundle, J. Geom. Phys. 6 (1989) 215-235 MR1040392

[2] L Álvarez-Cónsul, M García-Fernández, O García-Prada, Gravitating vortices, in preparation

[3] B Anchouche, I Biswas, Einstein-Hermitian connections on polystable principal bundles over a compact Kähler manifold, Amer. J. Math. 123 (2001) 207-228 MR1828221

[4] MF Atiyah, R Bott, The Yang-Mills equations over Riemann surfaces, Philos. Trans. Roy. Soc. London Ser. A 308 (1983) 523-615 MR702806

[5] T Aubin, Some nonlinear problems in Riemannian geometry, Springer, Berlin (1998) MR1636569

[6] A L Besse, Einstein manifolds, Ergeb. Math. Grenzgeb. 10, Springer, Berlin (1987) MR867684

[7] J-P Bourguignon, Invariants intégraux fonctionnels pour des équations aux dérivées partielles d'origine géométrique, from: "Partial differential equations, Part 1, 2", (B Bojarski, W Zajączkowski, B Ziemian, editors), Banach Center Publ. 27, Polish Acad. Sci., Warsaw (1992) 65-73 MR1205812 
[8] E Calabi, Extremal Kähler metrics, from: "Seminar on Differential Geometry", (ST Yau, editor), Ann. of Math. Stud. 102, Princeton Univ. Press (1982) 259-290 MR645743

[9] L Caporaso, A compactification of the universal Picard variety over the moduli space of stable curves, J. Amer. Math. Soc. 7 (1994) 589-660 MR1254134

[10] XX Chen, The space of Kähler metrics, J. Differential Geom. 56 (2000) 189-234 MR1863016

[11] XX Chen, Space of Kähler metrics, III: On the lower bound of the Calabi energy and geodesic distance, Invent. Math. 175 (2009) 453-503 MR2471594

[12] XX Chen, S K Donaldson, S Sun, Kähler-Einstein metrics and stability arXiv: 1210.7494

[13] X X Chen, G Tian, Geometry of Kähler metrics and foliations by holomorphic discs, Publ. Math. Inst. Hautes Études Sci. (2008) 1-107 MR2434691

[14] S K Donaldson, A new proof of a theorem of Narasimhan and Seshadri, J. Differential Geom. 18 (1983) 269-277 MR710055

[15] S K Donaldson, Anti self-dual Yang-Mills connections over complex algebraic surfaces and stable vector bundles, Proc. London Math. Soc. 50 (1985) 1-26 MR765366

[16] S K Donaldson, Infinite determinants, stable bundles and curvature, Duke Math. J. 54 (1987) 231-247 MR885784

[17] S K Donaldson, Remarks on gauge theory, complex geometry and 4-manifold topology, from: "Fields Medallists' lectures", (M F Atiyah, D Iagolnitzer, editors), World Sci. Ser. 20th Century Math. 5, World Sci. Publ. (1997) 384-403 MR1622931

[18] S K Donaldson, Symmetric spaces, Kähler geometry and Hamiltonian dynamics, from: "Northern California Symplectic Geometry Seminar", (Y Eliashberg, D Fuchs, T Ratiu, A Weinstein, editors), Amer. Math. Soc. Transl. Ser. 2 196, Amer. Math. Soc. (1999) 13-33 MR1736211

[19] S K Donaldson, Scalar curvature and projective embeddings, I, J. Differential Geom. 59 (2001) 479-522 MR1916953

[20] S K Donaldson, P B Kronheimer, The geometry of four-manifolds, The Clarendon Press, New York (1990) MR1079726

[21] A Fujiki, Moduli space of polarized algebraic manifolds and Kähler metrics, Sugaku Expositions 5 (1992) 173-191 MR1207204

[22] A Futaki, An obstruction to the existence of Einstein Kähler metrics, Invent. Math. 73 (1983) 437-443 MR718940

[23] A Futaki, Asymptotic Chow semi-stability and integral invariants, Internat. J. Math. 15 (2004) 967-979 MR2106156 
[24] A Futaki, H Ono, Einstein metrics and GIT stability, Sūgaku 60 (2008) 175-202 MR2422701

[25] M García-Fernández, Coupled equations for Kähler metrics and Yang-Mills connections, PhD thesis, Instituto de Ciencias Matemáticas, Madrid (2009) arXiv: 1102.0985

[26] M García-Fernández, C Tipler, Deformation of complex structures and the coupled Kähler-Yang-Mills equations arXiv:1301.4480

[27] D Gieseker, I Morrison, Hilbert stability of rank-two bundles on curves, J. Differential Geom. 19 (1984) 1-29 MR739780

[28] M Gromov, Pseudoholomorphic curves in symplectic manifolds, Invent. Math. 82 (1985) 307-347 MR809718

[29] Y-J Hong, Constant Hermitian scalar curvature equations on ruled manifolds, J. Differential Geom. 53 (1999) 465-516 MR1806068

[30] Y-J Hong, Stability and existence of critical Kaehler metrics on ruled manifolds, J. Math. Soc. Japan 60 (2008) 265-290 MR2392011

[31] L Huang, On joint moduli spaces, Math. Ann. 302 (1995) 61-79 MR1329447

[32] J Keller, C W Tønnesen-Friedman, Nontrivial examples of coupled equations for Kähler metrics and Yang-Mills connections, Cent. Eur. J. Math. 10 (2012) 1673-1687 MR2949645

[33] G Kempf, L Ness, The length of vectors in representation spaces, from: "Algebraic geometry”, (K Lønsted, editor), Lecture Notes in Math. 732, Springer, Berlin (1979) 233-243 MR555701

[34] S Kobayashi, Differential geometry of complex vector bundles, Publications of the Mathematical Society of Japan 15, Princeton Univ. Press (1987) MR909698

[35] S Kobayashi, K Nomizu, Foundations of differential geometry, Vol. I, John Wiley, New York (1963) MR0152974

[36] S Kobayashi, K Nomizu, Foundations of differential geometry, Vol. II, Interscience Tracts in Pure and Applied Mathematics 15, John Wiley, New York (1969) MR0238225

[37] C LeBrun, The Einstein-Maxwell equations, extremal Kähler metrics, and SeibergWitten theory, from: "The many facets of geometry", (O García-Prada, J-P Bourguignon, S Salamon, editors), Oxford Univ. Press (2010) 17-33 MR2681684

[38] C LeBrun, S R Simanca, On the Kähler classes of extremal metrics, from: "Geometry and global analysis”, (T Kotake, S Nishikawa, R Schoen, editors), Tohoku Univ., Sendai (1993) 255-271 MR1361191

[39] C LeBrun, S R Simanca, Extremal Kähler metrics and complex deformation theory, Geom. Funct. Anal. 4 (1994) 298-336 MR1274118 
[40] J Li, S-T Yau, The existence of supersymmetric string theory with torsion, J. Differential Geom. 70 (2005) 143-181 MR2192064

[41] T Mabuchi, K-energy maps integrating Futaki invariants, Tohoku Math. J. 38 (1986) 575-593 MR867064

[42] T Mabuchi, Some symplectic geometry on compact Kähler manifolds, I, Osaka J. Math. 24 (1987) 227-252 MR909015

[43] J E Marsden, G Misiołek, J-P Ortega, M Perlmutter, T S Ratiu, Hamiltonian reduction by stages, Lecture Notes in Math. 1913, Springer, Berlin (2007) MR2337886

[44] M Maruyama, Moduli of stable sheaves, II, J. Math. Kyoto Univ. 18 (1978) 557-614 MR509499

[45] D McDuff, D Salamon, Introduction to symplectic topology, 2nd edition, The Clarendon Press, New York (1998) MR1698616

[46] R Pandharipande, A compactification over $\bar{M}_{g}$ of the universal moduli space of slope-semistable vector bundles, J. Amer. Math. Soc. 9 (1996) 425-471 MR1308406

[47] A Ramanathan, Stable principal bundles on a compact Riemann surface, Math. Ann. 213 (1975) 129-152 MR0369747

[48] A Ramanathan, S Subramanian, Einstein-Hermitian connections on principal bundles and stability, J. Reine Angew. Math. 390 (1988) 21-31 MR953674

[49] I Mundet i Riera, A Hitchin-Kobayashi correspondence for Kähler fibrations, J. Reine Angew. Math. 528 (2000) 41-80 MR1801657

[50] G Schumacher, M Toma, Moduli of Kähler manifolds equipped with Hermite-Einstein vector bundles, Rev. Roumaine Math. Pures Appl. 38 (1993) 703-719 MR1263214

[51] S Semmes, Complex Monge-Ampère and symplectic manifolds, Amer. J. Math. 114 (1992) 495-550 MR1165352

[52] I M Singer, The geometric interpretation of a special connection, Pacific J. Math. 9 (1959) 585-590 MR0111062

[53] J Stoppa, Twisted constant scalar curvature Kähler metrics and Kähler slope stability, J. Differential Geom. 83 (2009) 663-691 MR2581360

[54] A Teleman, Symplectic stability, analytic stability in non-algebraic complex geometry, Internat. J. Math. 15 (2004) 183-209 MR2055369

[55] G Tian, K-stability and Kähler-Einstein metrics arXiv:1211.4669

[56] G Tian, Canonical metrics in Kähler geometry, Birkhäuser, Basel (2000) MR1787650

[57] G Tian, S-T Yau, Kähler-Einstein metrics on complex surfaces with $C_{1}>0$, Comm. Math. Phys. 112 (1987) 175-203 MR904143

[58] K Uhlenbeck, S-T Yau, On the existence of Hermitian-Yang-Mills connections in stable vector bundles, Comm. Pure Appl. Math. 39 (1986) S257-S293 MR861491 Corrected in 42 (1989) 703-707 
[59] X Wang, Moment map, Futaki invariant and stability of projective manifolds, Comm. Anal. Geom. 12 (2004) 1009-1037 MR2103309

[60] Y S Yang, Prescribing topological defects for the coupled Einstein and abelian Higgs equations, Comm. Math. Phys. 170 (1995) 541-582 MR1337133

[61] S-T Yau, On the Ricci curvature of a compact Kähler manifold and the complex Monge-Ampère equation, I, Comm. Pure Appl. Math. 31 (1978) 339-411 MR480350

LA-C, OG-P: Instituto de Ciencias Matemáticas

C/ Nicolás Cabrera 13-15, Campus Cantoblanco, 28049 Madrid, Spain

MG-F: MATHGEOM, École Polytechnique Fédéral de Lausanne

MA B1 437, Station 8, CH-1015 Lausanne, Switzerland

1.alvarez-consul@icmat.es, mariogf@imf.au.dk, mario.garcia@epfl.ch, oscar.garcia-prada@icmat.es

Proposed: Simon Donaldson

Received: 29 June 2012

Seconded: Richard Thomas, Frances Kirwan

Revised: 26 March 2013 